\title{
Introducing sequential aza-amino acids units induces repeated $\beta$-turns and helical conformations in peptides
}

\author{
Nicolo Tonali, ${ }^{a}$ Isabelle Correia, ${ }^{b}$ Jacopo Lesma, ${ }^{a}$ Guillaume Bernadat, ${ }^{a}$ Sandrine Ongeri, ${ }^{\text {a }}$ Olivier Lequin*
}

\author{
${ }^{a}$ Université Paris-Saclay, CNRS, BioCIS, 92290 Châtenay-Malabry, France. \\ E-mail: sandrine.ongeri@universite-paris-saclay.fr \\ ${ }^{b}$ Sorbonne Université, Ecole Normale Supérieure, PSL University, CNRS, Laboratoire des Biomolécules, 4 place Jussieu, 75252 Paris Cedex 05, France. \\ Email: olivier.lequin@sorbonne-universite.fr \\ Electronic Supplementary Information (ESI) available: \\ Synthesis and characterization of 1,3 and 5. NMR data of compounds 1-5. Crystallographic data for 2 . Molecular mechanics and DFT calculations. \\ See DOI: $10.1039 /$ c9ob02654a
}

A major current issue in medicinal chemistry is the design of small peptide analogues resistant to proteolysis and able to adopt preferential conformations, while preserving the selectivity and efficiency of natural peptides. Whereas the introduction of one aza-Gly in peptides has proven numerous biological and structural interest, the conformational effect of sequential aza-Gly or aza-amino acids bearing side chains has not been investigated. In this work, experimental NMR and X-ray data together with in silico conformational studies reveal that the introduction of two consecutive aza-amino acids in pseudotripeptides induces the formation of stable hydrogen-bonded $\beta$-turn structures. Notably, this stabilization effect relies on the presence of side chains on aza-amino acids, as more flexible conformations are observed with aza-Gly residues. Remarkably, a longer aza/aza/ $\alpha / a z a / a z a / \alpha$ pseudohexapeptide containing substituted aza-amino acids adopts repeated $\beta$-turns conformations which interconvert with a fully helical structure mimicking a $3_{10}$ helix.

\section{Introduction}

Peptide-based drugs have gained tremendous interest in the last two decades, reaching more than 60 approvals as commercial drugs and about 140 in clinical trials. ${ }^{1}$ However, one major current issue is the design of small peptide analogues able to adopt preferential conformations while possessing side chains similar to natural peptides in order to preserve their unique selectivity. The introduction of one aza-aminoacid in peptides, in order to afford the socalled aza-peptides, has shown significant success in providing biologically active peptides. ${ }^{2-6}$ Indeed, $^{\text {the }}$ substitution of the $\alpha-\mathrm{CH}(\mathrm{R})$ of at least one amino-acid residue by a nitrogen atom can increase drug efficiency, selectivity, and stability towards proteolytic degradation compared to the parent natural peptide. ${ }^{2-6}$ Among the numerous examples of peptides containing one aza-amino acid, Goserelin (Zoladex) has been approved as drug for the treatment of prostate cancer. It contains an aza-glycine (aGly), as most often encountered in representative azapeptides. Although peculiar conformational properties of aza-amino acids are claimed, a limited number of computational and experimental studies have been reported. The introduction of an $\alpha$-nitrogen atom generates two structural elements, hydrazine and urea. Computational analysis of 1,2-diformylhydrazine indicated that the stable structures are non-planar at nitrogen and show twisted conformation around the $\mathrm{N}-\mathrm{N}$ bond, with a favored $\phi$ dihedral angle around $\pm 90^{\circ} .^{7}$ The planar arrangement of the urea moiety constrains the $\psi$ dihedral angle around $0^{\circ}$ or $180^{\circ}$ (Figure 1). The propensity of small peptides containing one aza-amino acid to adopt a $\beta$-turn conformation has been mainly described. ${ }^{8}$ Zhou et al investigated the capacity of oligomers of mixed $\alpha / a z a / \alpha$ pseudotripeptides to adopt a $i, i+2$ or $i, i+3$ hydrogen-bonded helical conformation. ${ }^{9}$ Otherwise Zhang et al. demonstrated that the substitution of Gly with aGly in collagen provides an extra $\mathrm{H}$-bond donor that can lead to a hyperstable collagen triple helix. ${ }^{10}$<smiles>[R]N(NC(C)=O)C(=O)NC</smiles><smiles>[R]N(CC(=O)NC(C)=O)NC(=O)NC(C)=O</smiles> 
However, it appears that the conformation of peptides containing consecutive aza-amino acids has not been investigated so far. In this work, we describe experimental (NMR, X-ray) and in silico conformational studies of three tripeptide analogues containing two consecutive aza-amino acids, the aza/aza/ $\alpha$ pseudotripeptides $\mathbf{2 - 4}$, and of one natural tripeptide 1. This study brings new insight about the conformational effects of aza-substitution in peptide backbone and of side chain introduction on aza-amino acids (aAla, aVal). This led us to synthesize and investigate the conformational preferences of the foldamer aza/aza/ $\alpha / a z a / a z a / \alpha$ pseudohexapeptide $\mathbf{5}$, that is the dimer analogue of aza/aza/ $\alpha$ pseudotripeptide 2 .<smiles>CC(NC(=O)[C@H](NC(=O)OC(C)(C)C)C(C)C)C(=O)N[C@H](C(N)=O)C(C)C</smiles>
Boc-Val-Ala-Val- $\mathrm{NH}_{2} 1$<smiles>[R]C(=O)C(NC(=O)N(C)NC(=O)N(NC(=O)OC(C)(C)C)C(C)C)C(C)C</smiles>

$\mathrm{R}=\mathrm{NH}_{2} \quad$ Boc-aVal-aAla-Val- $\mathrm{NH}_{2} 2$ $\mathrm{R}=\mathrm{OBn} \quad$ Boc-aVal-aAla-Val-OBn 3<smiles>CC(C)C(NC(=O)N(C)NC(=O)N(NC(=O)OC(C)(C)C)C(C)C)C(=O)NN(C(=O)NN(C)C(=O)N[C@H](C(=O)N[C@@H](C(N)=O)C(C)C)C(C)C)C(C)C</smiles>

Boc-aVal-aAla-Val-aVal-aAla-Val- $\mathrm{NH}_{2} 5$

Fig. 2 Structure of the tripeptide containing three natural L- $\alpha$-amino acids $\mathbf{1}$, of the aza/aza/ $\alpha$ pseudotripeptides $\mathbf{2 - 4}$, and of the aza/aza/ $\alpha /$ aza/aza/ $\alpha$ pseudohexapeptide 5 .

\section{Results and discussion}

The synthesis of the tripeptide analogues containing two consecutive aza-amino acids Boc-aVal-aAla-Val-NH 2 and Boc-aGly-aGly-Val- $\mathrm{NH}_{2} 4$ has been recently reported by us. ${ }^{11,12}$ Only very rare examples of the incorporation of two or more consecutive aza-amino acids were reported to date, and we recently established some general guidelines regarding the activation and the coupling of alkyl-hydrazides either mutually or with a natural amino acid. ${ }^{11}$ While the incorporation of aGly is rather efficient from hydrazine moieties, the introduction of aza-amino acids bearing side chains must take into account the nucleophilicity and the nature of the alkyl-hydrazides. The syntheses of BocVal-Ala-Val- $\mathrm{NH}_{2}$ 1, Boc-aVal-aAla-Val-OBn 3, and Boc-aVal-aAla-Val-aVal-aAla-Val-NH $\mathbf{5}$ are described in detail in supplementary information. Boc-Val-Ala-Val- $\mathrm{NH}_{2} \mathbf{1}$ was prepared using classical peptide coupling methods. BocaVal-aAla-Val-OBn 3 was prepared following the same procedure as for the synthesis of its $C$-terminal amide analogue $\mathbf{2}$ described in ref 11 . It is based on the activation of Cbz-1-methyl-hydrazine using 4nitrophenylchloroformate (93\% yield) and on the subsequent reaction with Boc-2-isopropyl-hydrazine in order to obtain the diazapeptide in good yield (80\%). The successive cleavage of the $\mathrm{Cbz}$ group followed by the reaction with the activated Val-OBn (using 4-nitrophenylchloroformate) afforded compound $\mathbf{3}$ in satisfactory yield (66\%, Scheme S2). Boc-aVal-aAla-Val-OBn 3 was then either deprotected at its $C$-terminus by hydrogenolysis or at its $N$-terminus by acid cleavage to obtain the two intermediates that were coupled to give, after the replacement of the benzyl ester group by the $C$-terminal amide, the desired pseudohexapeptide Boc-aVal-aAla-Val-aVal-aAla-Val-NH 25 (Scheme S2).

As some compounds exhibited low solubility in water, NMR conformational analyses were run in $\mathrm{CD}_{3} \mathrm{OH}$. This protic solvent allowed us to get as close as possible to an aqueous environment and also to record NMR spectra at low temperatures (248-273 K range), in order to better characterize slowly interconverting conformers. Different NMR parameters were examined to analyze the conformational propensities of compounds 1-5, in particular interproton ROE correlations, temperature coefficient $\left(\Delta \delta_{\mathrm{HN}} / \Delta \mathrm{T}\right)$ of the amide protons, and vicinal ${ }^{3} \mathrm{JN}_{\mathrm{HN} \alpha}$ coupling constants of the natural amino acids.

As expected for a short linear peptide containing only natural $\alpha$-amino acids, tripeptide Boc-Val-Ala-Val-NH $\mathbf{B}_{2} \mathbf{~ w a s}$ found to be highly disordered. Vicinal ${ }^{3} \mathrm{~J}_{\mathrm{HN}-\mathrm{H \alpha}}$ coupling constant analysis shows that the central Ala residue is flexible $\left({ }^{3} J_{\mathrm{HN}-\mathrm{H} \alpha}\right.$ of $\left.6.6 \mathrm{~Hz}\right)$ while the two flanking Val residues preferentially adopt extended conformations $\left({ }^{3} J_{\mathrm{HN}-\mathrm{H} \alpha}\right.$ of $8.7 \mathrm{~Hz}$ and $8.6 \mathrm{~Hz}$, reflecting preferred $\varphi$ angle values in the $-150^{\circ}$ to $-90^{\circ}$ range), as expected for $\beta$-branched aliphatic 
residues (Table S3). Strong sequential and medium intraresidual $\mathrm{H}^{\alpha}-\mathrm{HN}$ ROE correlations also support mostly extended conformations (Figure S3). The highly negative temperature coefficients of all amide protons (Table 1 ) confirms solvent exposure and the absence of stable intramolecular hydrogen bonds.

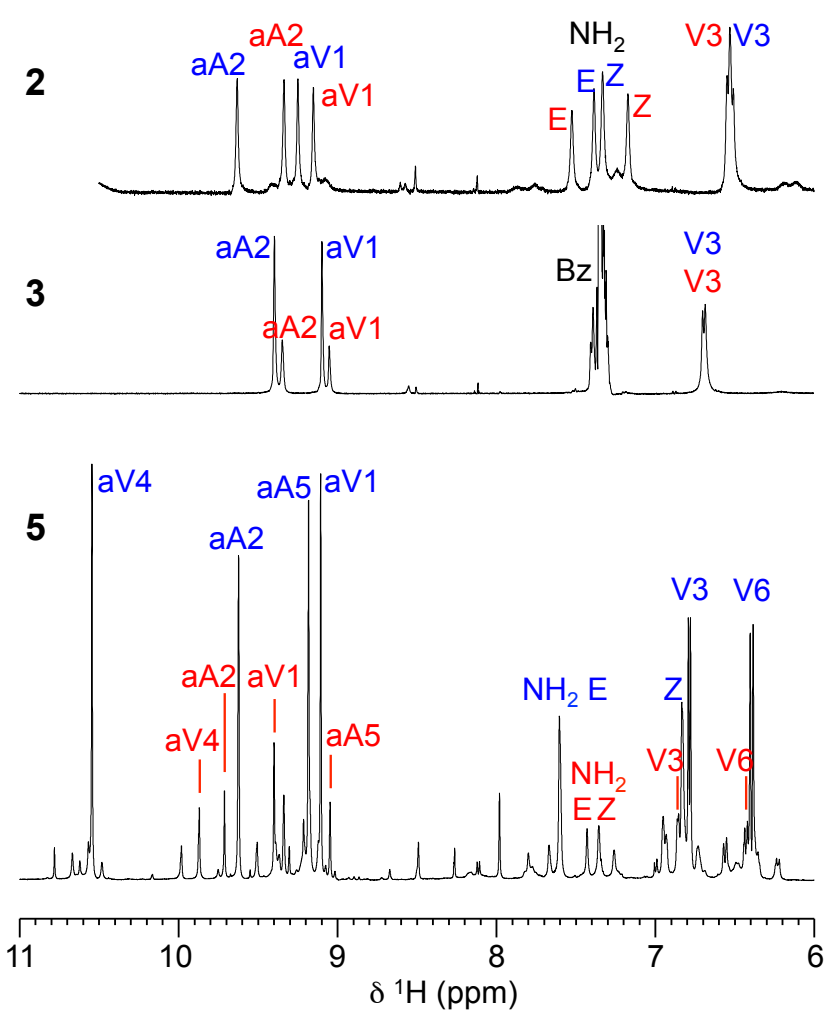

Fig. $31 \mathrm{D}{ }^{1} \mathrm{H}$ NMR spectra of compounds $\mathbf{2}, 3$ and $\mathbf{5}$ in methanol at $273 \mathrm{~K}$, showing the region of HN signals. The assignments of the two most-populated forms for each compound are indicated by one-letter amino acid code (aX corresponds to aza-amino acids).

Table 1. Amide proton temperature coefficients $\Delta \delta_{H N} / \Delta T$ of compounds 1-5 in methanol. The two most-populated conformers observed for compounds $\mathbf{2}, \mathbf{3}$ and $\mathbf{5}$ are indicated by $\mathrm{A}$ and $\mathrm{B}$ letters. Temperature coefficients above $-4.5 \mathrm{ppb} /{ }^{\circ} \mathrm{C}$ are indicated in bold.

\begin{tabular}{|c|c|c|c|c|c|c|c|c|c|c|c|c|}
\hline Res & 1 & Res & $2 A$ & 2B & Res & $3 A$ & 3B & Res & 4 & Res & $5 A$ & 5B \\
\hline Val1 & -8.5 & aVal1 & -7.3 & -7.1 & aVal1 & -7.0 & -6.9 & aGly1 & -9.4 & aVal1 & -6.1 & -6.5 \\
\hline Ala2 & -7.7 & aAla2 & -6.6 & -6.8 & aAla2 & -7.8 & -7.7 & aGly2 & -6.3 & aAla2 & -5.0 & -6.3 \\
\hline Val3 & -8.4 & Val3 & -2.6 & -2.3 & Val3 & -3.9 & -3.9 & Val3 & -4.3 & Val3 & -2.9 & -3.0 \\
\hline $\mathrm{NH}_{2} \mathrm{Z}$ & -7.1 & $\mathrm{NH}_{2} \mathrm{Z}$ & -8.4 & -7.3 & & & & $\mathrm{NH}_{2} \mathrm{Z}$ & -8.6 & aVal4 & -6.3 & -4.5 \\
\hline \multirow[t]{4}{*}{$\mathrm{NH}_{2} E$} & -8.3 & $\mathrm{NH}_{2} E$ & -3.3 & -6.2 & & & & $\mathrm{NH}_{2} E$ & -6.1 & aAla5 & -2.8 & -3.6 \\
\hline & & & & & & & & & & Val6 & -1.8 & -2.2 \\
\hline & & & & & & & & & & $\mathrm{NH}_{2} \mathrm{Z}$ & -5.5 & -8.8 \\
\hline & & & & & & & & & & $\mathrm{NH}_{2} E$ & -5.8 & -3.8 \\
\hline
\end{tabular}

Unlike natural peptide 1, the diaza-peptide analogue Boc-aVal-aAla-Val- $\mathrm{NH}_{2} \mathbf{2}$ was characterized by a markedly different conformational space. An equilibrium between two major conformers (45\% population for each) was observed by NMR in methanol (Figures 3 and S6). Two additional minor forms were also observed (population < 5\%) and were ascribed to the cis-trans isomerization of the Boc protective group (a feature that was also observed for tripeptide 1). Both major conformers show similar long-range ROEs between the tert-butyl protons of Boc with the amide proton and side chain methyl protons of the Val residue, indicative of folded conformations (Figure 4A). In both conformers, the amide protons of aVal1 and aAla2 residues exhibited strong variations of their temperature coefficient (Table 1), as expected for solvent-exposed groups, whereas the $\mathrm{NH}$ of Val3 has a small temperature dependence $\left(\Delta \delta \mathrm{HN} / \Delta \mathrm{T}\right.$ higher than $\left.-4.5 \mathrm{ppb} /{ }^{\circ} \mathrm{C}\right)$ suggesting its engagement in a stable $\mathrm{H}$-bond. One of the two 
protons of the carboxamide group (assigned as $\mathrm{H}_{E}$ ) also showed a small variation, in conformer $\mathrm{A}$ only (Table 1). Thus, an equilibrium is suggested between two conformations that differ in the spatial orientation of the $\mathrm{C}$-terminal carboxamide, leading to a double turn or single turn structure, in conformers $A$ and $B$ respectively.

Conformers $A$ and $B$ exhibit similar sets of ROEs (Figure 4A), with the exception of an additional ROE observed in conformer $\mathrm{A}$ between $\mathrm{Val} \mathrm{HN}$ proton and $\mathrm{H}_{\mathrm{E}}$ carboxamide proton. For each conformer, strong sequential $\mathrm{HNi} / \mathrm{HNi}+1$ ROEs were observed, indicative of $\psi$ dihedral angles around $0^{\circ}$, and $\phi$ dihedral angles around $\pm 90^{\circ}$ for aza-amino acid backbone.

NMR structure calculations indicated that folded hydrogen-bonded conformations can only occur if the two consecutive aza-amino acids adopt similar signs for their $\phi$ angle. NMR structure calculations refined by DFT (Figure 4B) revealed that conformer A corresponds to a double turn structure in which all residues have negative $\phi$ angles.

The alternative conformer $B$ is characterized by positive $\phi$ values for the two aza-amino acids and a negative $\phi$ value for Val3. Thus the conformational equilibrium observed by NMR corresponds to slow exchange within the two azaamino acid units, interconverting from $-/-$ to $+/+\phi$ angles. Forms $A$ and $B$ have conformations close to regular types I and I' $\beta$-turns, respectively. When all residues have negative $\phi$ values (conformer $A$ ), the backbone folds in a righthanded helical turn allowing the carboxamide group to be engaged in an additional H-bond with aVal1 carbonyl group. This $\mathrm{H}$-bond formation is no longer possible when the two aza-aminoacid units change handedness (conformer $\mathrm{B}$ ), as the $\mathrm{L}-\mathrm{V}$ al3 residue still adopts a favored negative $\phi$ angle.

A

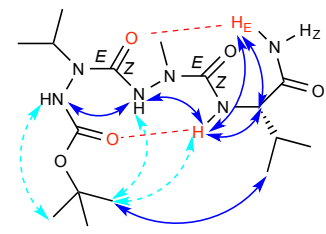

conformer A

B

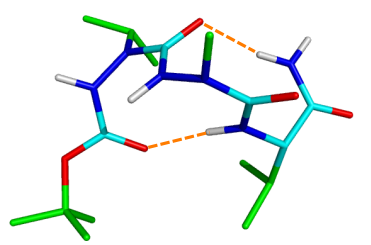

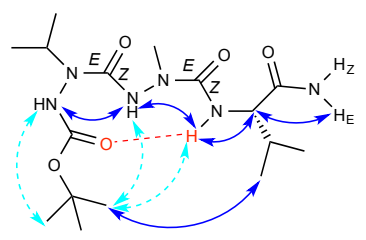

conformer B

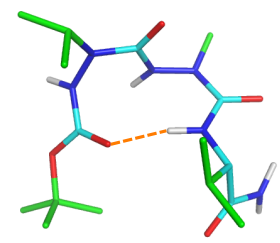

C

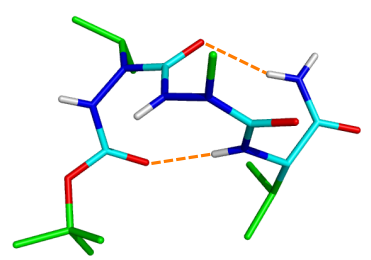

Fig. 4 Conformations of Boc-aVal-aAla-Val- $\mathrm{NH}_{2}$ 2. A) NMR conformational parameters in methanol: schematic representation of ROEs (blue and dashed cyan arrows correspond to strong, and weak intensity ROEs, respectively), and of hydrogen bonds (in red) as inferred from amide proton temperature coefficients. B) Corresponding NMR structures calculated from distance restraints using AMBER and refined by DFT with methanol PCM solvation model. The $(\phi, \psi)$ angles of aVal1 and aAla2 are $\left(-73^{\circ},-10^{\circ}\right)$ and $\left(-64^{\circ},-16^{\circ}\right)$ in conformer $A$, and $\left(+71^{\circ}\right.$, $\left.+16^{\circ}\right)$ and $\left(+81^{\circ},+7^{\circ}\right)$ in conformer B. C) X-ray structure showing one of the two chains of the asymmetric unit. The average $(\phi, \psi)$ angles of $X$-ray chains are: $\left(-62^{\circ},-19^{\circ}\right)$ for aVal1, $\left(-77^{\circ},-7^{\circ}\right)$ for aAla2 and $\left(-65^{\circ},-23^{\circ}\right)$ for Val3. The N $\alpha$ atoms have a small pyramidal character, as assessed by the $11^{\circ}$ angular deviation between $\mathrm{N}-\mathrm{C} \beta-\mathrm{C}^{\prime}$ and $\mathrm{N}-\mathrm{C} \beta-\mathrm{N} \alpha$ planes. The DFT refinement of NMR structures yielded good description of the pyramidal character, with an angular deviation of $12^{\circ}$ (vs. $3^{\circ}$ before refinement).

Diaza-peptide $\mathbf{2}$ was amenable to crystallisation and X-ray diffraction data revealed a double turn structure stabilized by two intramolecular hydrogen bonds, which is very similar to conformer A characterized by NMR in solution (Figures 4C, S16 and S17). Structures of compounds were also studied by Monte Carlo conformational search using GBSA water solvation model, followed by refinement using DFT. The objective of the simulations was double: firstly, evaluate our ability to predict the conformations of peptides containing aza-amino acids and the rotameric states of urea moieties by in silico tool; secondly, better anticipate the peptide conformations in a solvent closer to physiological conditions by using a water solvation model. The DFT refinement enabled us to better take into account the weak pyrimidalization of the nitrogen atoms in aza-amino acids that was slightly underestimated in 
molecular mechanics force fields. Computational studies were fully consistent with the NMR experimental data, with a predominance of conformers showing extended or semi-folded structures for the natural peptide $\mathbf{1}$ (Tables S17 and S18), and folded turn structures for diaza-peptide 2. A very good agreement was observed with the X-ray structure of $\mathbf{2}$ (Figure S19).

We next studied the diaza-peptide analogue Boc-aVal-aAla-Val-OBn 3 having a $C$-terminal benzyl ester instead of a carboxamide as in $\mathbf{2}$, which therefore lacks a second $\mathrm{H}$-bond donor group. Diaza-peptide $\mathbf{3}$ exhibits a similar conformational equilibrium in solution and adopts single hydrogen bonded $\beta$-turn structures, as revealed by ROEs and amide bond temperature coefficients (Table 1, Figure S8). However, the equilibrium is shifted toward one conformer (7:3). This shows that, irrespective of $\mathrm{H}$-bond formation, the presence of a chiral L-amino acid induces a conformational preference for one $\beta$-turn folding type (I vs I') within the achiral diaza-peptide unit. Computational studies suggest that the most stable conformation is type l' $\beta$-turn, corresponding to $+/+\phi$ angles for aza-amino acids (Table S16).

The influence of aza-amino acid side chain was investigated by examining tripeptide Boc-aGly-aGly-Val-NH $\mathbf{H}_{2}$. $\mathrm{NMR}^{-}$ spectra showed faster backbone rotation of aGly units with respect to substituted aza-amino acids as a single set of resonance was observed at room temperature. However conformational isomers in slow exchange could be detected by lowering the temperature. The amide proton of Val3 has a medium temperature coefficient (Table 1), and a weak ROE with Boc protons, indicating a weak propensity to populate $\beta$-turn conformations. MM/DFT calculations of Boc-aGly-aGly-Val- $\mathrm{NH}_{2} 4$ (Tables S17-S18) showed a wider conformational space that encompasses more extended conformations. In particular, the absence of side chain allows aGly residue to populate two minima corresponding to $\psi$ dihedral angles around $0^{\circ}$ or $180^{\circ}$. In contrast, in substituted aza-amino acids, the urea motif has a preferred $E, Z$ geometry in all low energy conformers, with $\psi$ dihedral angles around $0^{\circ}$.

Thus, unlike the corresponding natural tripeptide $\mathbf{1}$ and pseudotripeptide $\mathbf{4}$ containing two aGly which turned out to be preferentially extended, aza/aza/ $\alpha$ pseudotripeptides containing aza-amino acids with side chains have the tendency to adopt 10-membered $\beta$-turn conformations, with a hydrogen bond between the carbonyl group of the Boc group, mimicking a residue $\mathrm{i}$ and the amide proton of residue $\mathrm{i}+3$. The $\mathrm{C}$-terminal primary amide, mimicking the possible presence of an amide bond with a fourth residue, imparts to the backbone of the aza/aza/ $\alpha$ pseudotripeptides the possibility to establish a second i, i+3 hydrogen bond offering two 10-membered cycles, provided that aza-amino acids adopt negative $\phi$ angles like the L-amino acid.

As the helical conformation propensity of Boc-aVal-aAla-Val- $\mathrm{NH}_{2} \mathbf{2}$ was demonstrated, we envisioned the possibility to form a pseudo $3_{10}$-helix conformation with its dimer analogue Boc-aVal-aAla-Val-aVal-aAla-Val-NH 2 5. The NMR spectra of pseudo hexapeptide $\mathbf{5}$ in methanol exhibited very high complexity. Indeed, at least eight sets of resonances could be detected, corresponding to different conformational isomers in slow exchange (Figure 3 ). The population of the two most populated forms reaches $55 \%$ and $15 \%$, the other minor forms being far less populated (7\% or less).

All aza-amino acids in the different forms have strong sequential HNi/HNi+1 ROEs with the following residue, compatible with $(\phi, \psi)$ angles around $\left( \pm 90^{\circ}, 0^{\circ}\right)$. This suggests that the conformational equilibria likely correspond to slow rotation between $-90^{\circ}$ and $+90^{\circ} \phi$ dihedral angles. The temperature coefficients of amide protons could be measured for the two most populated species (Table 1). Interestingly, several amide protons have small temperature dependence of their chemical shifts, supporting the formation of $\mathrm{H}$-bonded turns in the different conformational isomers of hexapeptide 5. In particular, the major form shows evidence of $\mathrm{H}$-bond involvement for HN groups of Val3, aAla5 and Val6, but not aVal4. Accordingly, this conformer shows several $i, i+2$ and $i, i+3$ ROE connectivities, consistent with the formation of several turn structures along the backbone (Figure 5A). NMR structure calculations converged toward a low energy conformer in which the aza-amino acids have $\phi$ dihedral angles around $+90^{\circ}$, corresponding to $\phi$ signs $+/+/-/+/+/-$ for the six residues (Figure 5B). The hexapeptide forms three $\mathrm{H}$-bonded turns, two type I' $\beta$-turns centered on diaza-peptide segments and a type II $\beta$-turn centered on Val3-aVal4. The repeated $\beta$-turn structure leads to a compact hairpin shape with close proximity of residues aAla2 and Val6 at the $N$ - and $C$-extremities, mimicking an $\alpha$-turn geometry. This compact conformation is fully consistent with the observation of several long-range ROEs between carboxamide $\mathrm{H}_{\mathrm{z}}$ proton of Val6 with Boc, aVal1 and aAla2 protons. The calculated structure is stabilized by an additional H-bond between the $\mathrm{CO}$ group of Val6 and $\mathrm{HN}$ of aAla2. However, this amide proton has average temperature dependence, suggesting a more transient $\mathrm{H}$-bond engagement. 


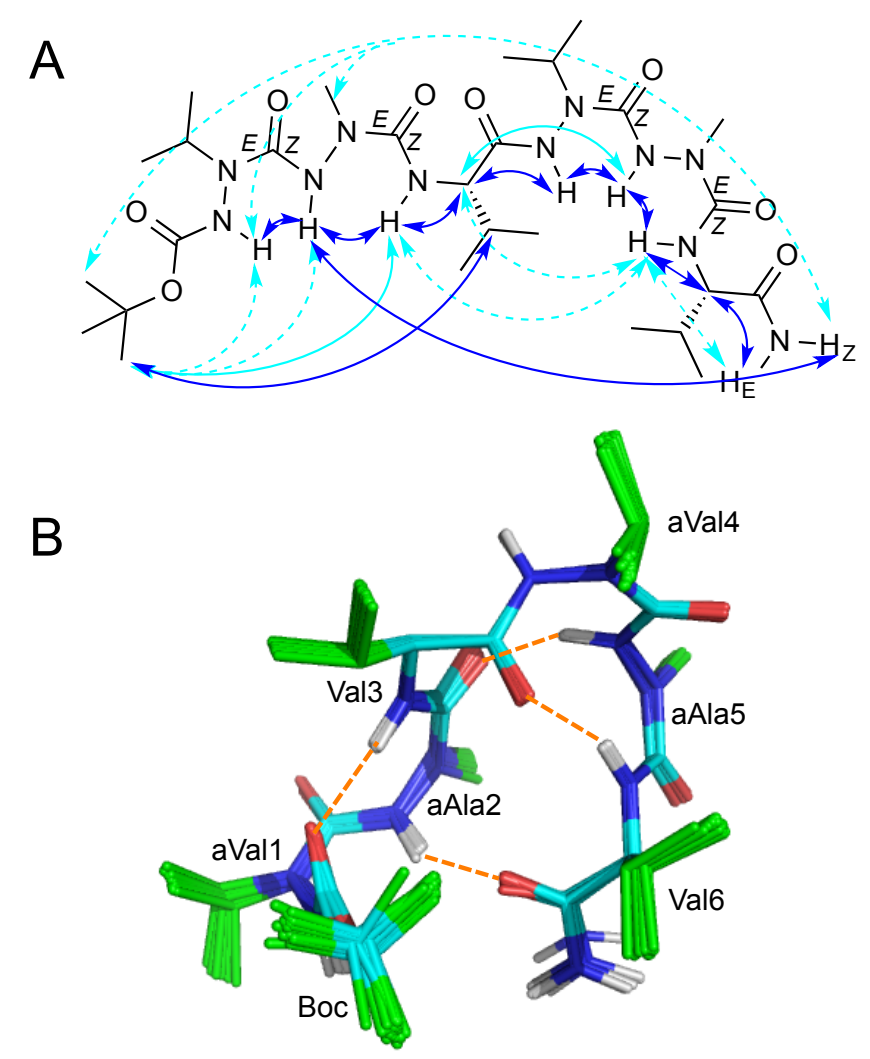

Fig. 5 Conformation of the major conformer of Boc-aVal-aAla-Val-aVal-aAla-Val-NH2 5A. A) ROEs observed in methanol: plain blue, plain cyan and dashed cyan arrows correspond to strong, medium and weak intensities, respectively. B) NMR structure showing the repeated turns structure. Hydrogen bonds are indicated by dashed orange lines.

A

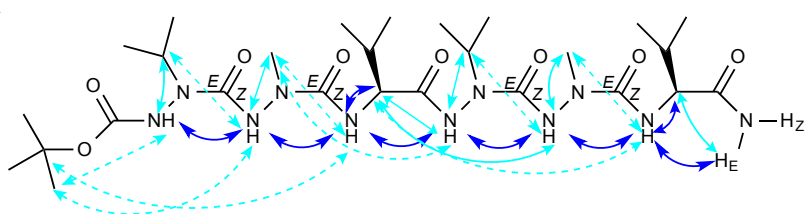

B

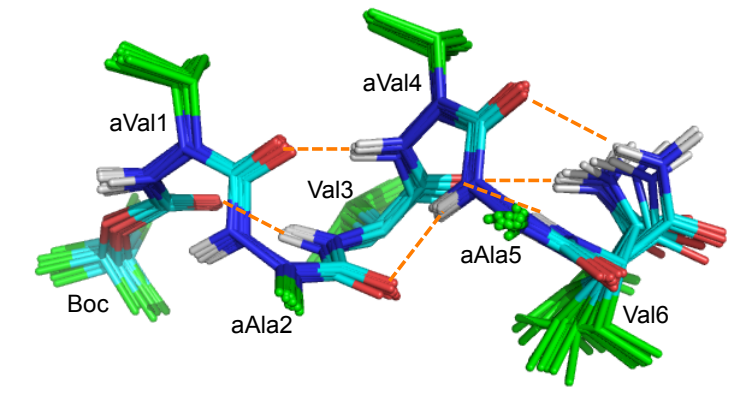

Fig. 6 Conformation of the second most-populated conformer of Boc-aVal-aAla-Val-aVal-aAla-Val-NH ${ }_{2}$ 5B. A) ROEs observed in methanol. B) NMR structure showing $3_{10}$-type helical folding. Hydrogen bonds are indicated by dashed orange lines. 
Interestingly, the second most-populated form is characterized by small temperature coefficients of HN protons for all residues within 3-6 segment (Table 1), supporting an uninterrupted $\mathrm{H}$-bond network, in contrast with the major conformer. This is consistent with full helix formation, which is confirmed by a sequential ROE between HN protons of Val3 and aVal4, which was not observed in the most-populated conformer (Figure $6 \mathrm{~A}$ ), and a smaller ${ }^{3} J_{\mathrm{HN}-\mathrm{H} \alpha}$ coupling constant for Val3 in agreement with a helical conformation $(5.2 \mathrm{~Hz}$ versus $6.5 \mathrm{~Hz}$ in the major conformer, Table S13). NMR structure calculation indicates that all residues adopt negative $\phi$ angles (-/-/-/-/-/- conformer). The fold is close to that of a regular $3_{10}$ helix with $i, i+3$ hydrogen bonding (Figure $6 \mathrm{~B}$ ).

The high complexity and spectral overlap of NMR spectra precluded complete assignment and full characterization of the minor conformational isomers of compound 5. However, structure calculations with a limited set of NMR distance restraints suggest that the main source of conformational heterogeneity arises from the interconversion of diaza-peptide units between $+/+$ and $-/-\phi$ angles. Four main topologies can be described (Figure S18), consisting of repeated $\beta$-turns of types $\mathrm{I}, \mathrm{I}^{\prime}$, or II, and potentially leading to partial or full helical fold.

\section{Conclusions}

In conclusion, this work brings the first structural data on peptides containing consecutive aza-amino acids, from combined NMR, X-ray and in silico MM/DFT studies. The twisted conformation around the N-N bonds of aza-amino acids induces favored $\phi$ dihedral angles around $\pm 90^{\circ}$. The presence of a side chain (aVal, aAla) further restricts the $\psi$ dihedral angles around $0^{\circ}$. In contrast to peptides made of $\alpha$-amino acids, these conformational restrictions confer unique properties on the aza/aza/ $\alpha$ pseudotripeptides giving them a strong tendency to adopt 10 -membered $\beta$-turn conformations. Due to the absence of chirality, the aza-aza moiety can adopt type I or the mirror image type I' $\beta$ turns, corresponding to $-/-$ or $+/+\phi$ angles, respectively. However, the equilibrium between types I and I' conformations is influenced by the chiral $\alpha$-amino-acid. The slow exchange within the two aza-amino acid units, interconverting from $-/-$ to $+/+\phi$ angles observed in solution is displaced in favor of one conformer at the solidstate as demonstrated in the X-ray diffraction data. Similarly, it can be assumed that this conformational equilibrium can be shifted upon interaction with a biological target. We have then explored the potentiality of aza/aza/ $\alpha$ bricks to adopt hydrogen-bonded structures in longer peptides, using a pseudohexapeptide foldamer aza/aza/ $\alpha / a z a / a z a / \alpha$. This compound was proven to adopt repeated $\beta$-turns or fully helical structures. Similar $\mathrm{i}$, $\mathrm{i}+3$ $\mathrm{C}=\mathrm{O} \cdots \mathrm{H}-\mathrm{N}$ hydrogen-bonded helices were reported by $\mathrm{S}$. Gellman et al. using backbones that contain pure $\beta$-residue oligomers (12-helix), and oligomers containing a 1:1 $\alpha: \beta$ repeat (11-helix), ${ }^{13}$ and either a $2: 1$ or a $1: 2$ repeating pattern of $\alpha$ - and $\beta$-amino acid residues $\left(12 / 11\right.$ or $10 / 11$ type helix). ${ }^{14} 12$-helix, 13 -helix and $\alpha$-helix-like conformation were also observed by S. Gellman et al. in $\alpha / \gamma, \beta / \gamma$ and $\alpha / \beta / \gamma$-peptides respectively. ${ }^{15,16,17}$ However, the $3_{10}$ helix observed in aza/aza/ $\alpha / a z a / a z a / \alpha$ is more comparable to $i, i+3$ hydrogen-bonded helices formed by pure $\alpha$-residue oligomers. Interestingly also, a hairpin structure was observed in the major conformer mimicking an $\alpha$-turn geometry. Very few molecules have been described to stabilize isolated $\alpha$-turn conformations and the strategy is essentially to replace the $\mathrm{i}, \mathrm{i}+4$ hydrogen bond by a covalent bond. ${ }^{18}$ As far as we know, this work provides the proof of concept that foldamers based on diaza-amino acids units might resolve a major issue in the use of peptides as drugs, by stabilizing turns or helical conformations similar to natural peptides and retaining the selectivity due to the lateral chains. This new class of foldamers provides valuable opportunities to explore them in many different areas and applications. In particular, the number of aza/aza/ $\alpha$ bricks and the nature of the side chains can be modulated and adapted to further promote helical structures, and to design helical mimetics as ligands of helical structures of major biological targets. We can explore them for example as selective inhibitors of protein-protein interactions. ${ }^{19,20}$ The adaptive chirality of the aza-aza subunit can be an advantage as it is recognized that compounds adopting several kinetically and thermodynamically accessible conformations might be much more powerful inhibitors of protein-protein interactions with respect to rigid ones. ${ }^{21,22}$ Helical mimetics can also afford various applications such as membrane active peptides possessing antimicrobial, cytolytic, or cell penetrating properties. ${ }^{23,24,25}$ 


\section{Experimental Section}

\section{Synthesis}

The synthesis and characterization of Boc-Val-Ala-Val- $\mathrm{NH}_{2}$ 1, Boc-aVal-aAla-Val-OBn 3, and Boc-aVal-aAla-Val-aVal-aAla-Val-NH2 5 are detailed in supporting information. Boc-aVal-aAla-Val- $-\mathrm{NH}_{2} \mathbf{2}$ and Boc-aGly-aGly-Val- $\mathrm{NH}_{2} \mathbf{4}$ were prepared according to our published methods. $^{11,12}$

\section{NMR spectroscopy}

NMR experiments were acquired on a Bruker NMR spectrometer operating at a ${ }^{1} \mathrm{H}$ frequency of $500.3 \mathrm{MHz}$ and equipped with either a room temperature TXI probe (compounds 1-4) or a cryogenic TCI probe (compound 5). NMR data were processed with Topspin 3.2 (Bruker) and $2 \mathrm{D}$ experiments were analysed with NMRFAM-SPARKY program. ${ }^{1} \mathrm{H}$ and ${ }^{13} \mathrm{C}$ chemical shifts were referenced to the solvent signal (residual protonated $\mathrm{CHD}_{2} \mathrm{OH}$ at $3.31 \mathrm{ppm}$ and deuterated ${ }^{13} \mathrm{CD}_{3} \mathrm{OH}$ at $49.0 \mathrm{ppm}$, respectively) and ${ }^{15} \mathrm{~N}$ chemical shifts were referenced indirectly. ${ }^{1} \mathrm{H},{ }^{13} \mathrm{C}$ and ${ }^{15} \mathrm{~N}$ resonance assignments were obtained from the analysis of $2 \mathrm{D}{ }^{1} \mathrm{H}-{ }^{1} \mathrm{H}$ TOCSY (mixing time of $70 \mathrm{~ms}$ ), $2 \mathrm{D}{ }^{1} \mathrm{H}-{ }^{1} \mathrm{H}$ ROESY (mixing times of $0.5-0.8 \mathrm{~s}$ ), $2 \mathrm{D}^{1} \mathrm{H}^{13} \mathrm{C}$ HSQC, $2 \mathrm{D}^{1} \mathrm{H}^{13}{ }^{13} \mathrm{C} \mathrm{HMBC}$, and $2 \mathrm{D}^{1} \mathrm{H}^{-15} \mathrm{~N} \mathrm{HSQC}$ spectra. The temperature gradients of the amide proton chemical shifts were derived from a series of $1 \mathrm{D}^{1} \mathrm{H}$ spectra recorded between $258 \mathrm{~K}$ and $313 \mathrm{~K}$ for compounds $1-4$, and from $2 \mathrm{D}{ }^{1} \mathrm{H}$ ${ }^{15} \mathrm{~N}$ HSQC spectra recorded between $272 \mathrm{~K}$ and $298 \mathrm{~K}$ for compound 5.

\section{NMR structure calculation}

Aza-amino acids were parameterized using gaff forcefield atom types and partial charges were computed with AM1-BCC method implemented in Antechamber. Structures of compounds $\mathbf{2}$ and $\mathbf{5}$ were calculated using Amber14 program and ff14SB forcefield, using NMR-restrained molecular dynamics, as previously described. ${ }^{26}$ Selected structures were refined without restraints by DFT at the B3LYPD3/cc-pVTZlevel in Gaussian 16 (see below), using PCM solvation model matched for methanol.

\section{Molecular mechanics and DFT calculations}

Arbitrary initial conformations of compounds 1-5 were energy-minimized according to the conjugate gradient method ${ }^{27}$ in the OPLS2005 force field ${ }^{28}$ combined with GBSA implicit water solvation model ${ }^{29}$ as implemented in the MacroModel v10.2 software package. ${ }^{30}$ The $^{2}$ convergence criterion was set to $0.05 \mathrm{~kJ} \cdot \mathrm{mol}^{-1} \cdot \AA^{-1}$ on the energy gradient. Resulting coordinates were then individually subjected to an unconstrained conformational search according to the MCMM (Monte Carlo Multiple Minima) method ${ }^{31}$ with similar parameters. 100,000 conformations were calculated and clustered by the centroid linkage method with a $0.66 \AA$ merge distance threshold.

Geometries of selected models were then optimized without constraint in the framework of the density functional theory ${ }^{32}$ using Becke three-parameter Lee-Yang-Parr exchange-correlation functional, ${ }^{33}$ Dunning's correlation consistent basis set of triple $\zeta$ valence quality, ${ }^{34}$ and the D3 version of Grimme's dispersion correction, ${ }^{35}$ in conjunction with the polarizable continuum solvation model parametrized for water, ${ }^{36}$ as implemented in the Gaussian 16, revision B.01 software package. ${ }^{37}$ Stationary points were qualified as energy minima by checking for the absence of imaginary frequency after performing vibrational analysis at the same level of theory upon geometrical convergence. Thermodynamic quantities at $298.15 \mathrm{~K}$ were calculated using the zero-point and thermal energy corrections derived from unscaled frequencies.

\section{Conflicts of interest}

There are no conflicts to declare.

\section{Acknowledgements}

The French Ministère de l'Enseignement Supérieur et de la Recherche (MESR) is thanked for the PhD fellowships for N. Tonali and J. Lesma. Karine Leblanc (Service d'Analyses-HPLC-Masse BioCIS, Univ. Paris Saclay) is thanked for HPLC and HRMS analyses. The DRX Platform of Sorbonne Universite is thanked for the crystallographic data. The Laboratory BioCIS is a member of the Laboratory of Excellence LERMIT supported by a Grant from ANR (ANR-10-LABX-33). 


\section{References}

1 (a) K. Fosgerau and T. Hoffmann, Drug Discov Today, 2015, 20, 122; (b) J. L. Lau and M.K. Dunn, Bioorg. Med. Chem., 2018, 26, 2700.

2 (a) C. Proulx , D. Sabatino , R. Hopewell, J. Spiegel, Y. García Ramos and W. D. Lubell, Future Med Chem., 2011, 3, 1139-1164; (b) A. Zega, Curr. Med. Chem., 2005, 12, 589-597; (c) A. Begum, D. Sujatha, K.V.S.R.G. Prasad and K. Bharathi, Asian J. Chem., 2017, 29,1879 .

3 (a) J. Lee and M. Bogyo, Bioorg. Med. Chem. Lett., 2012, 22, 1340; (b) A. J. Kasznel, Y. Zhang, Y. Hai, D. M. Chenoweth, J. Am. Chem. Soc., 2017, 139, 9427.

4 (a) R. Chingle, C. Proulx and W. D. Lubell, Acc. Chem. Res. 2017, 50, 1541; (b) F. M. Mir, N. D. Prasad Atmuri, C. B. Bourguet, J. Rodon Fores, X. Hou, S. Chemtob and W. D. Lubell, J. Med. Chem., 2019, 62, 4500.

5 J. T. Randolph, X. Zhang, P. P. Huang, L. L. Klein, K. A. Kurtz, A. K. Konstantinidis, W. He, W. M. Kati and D. J. Kempf, Bioorg. Med. Chem. Lett., 2008, 18, 2745.

6 M. Galibert, M. Wartenberg, F. Lecaille, A. Saidi, S. Mavel, A. Joulin-Giet, B. Korkmaz, D. Brömme, V. Aucagne, A. F. Delmas and G. Lalmanach, Eur. J. Med. Chem. 2018, 144, 201.

7 (a) M. Thormann and H.-J. Hofmann, J. Mol. Struct. (THEOCHEM), 1999, 469, 63; (b) F. Ramondo and L. Bencivenni, J. Chem. Soc., Perkin Trans. 2, 1995, 1797; (c) C. H. Reynolds and R. E. Hormann, J. Am. Chem. Soc., 1996, 118, 9395.

8 (a) H.-J. Lee, I.-A. Ahn, S. Ro, K.-H. Choi, Y.-S. Choi and K.-B. Lee, J. Peptide Res., 2000, 56, 35; (b) H.-J. Lee, H. M. Park and K.-B. Lee, J. Mol. Struct., 2001, 569, 43; (c) H.-J. Lee, K.-H. Choi, I.-A. Ahn, S. Ro, H. G. Jang, Y.-S. Choi and K.-B. Lee, Biophys. Chem., 2007, 125, 117; (d) C. Abbas, G. Pickaert, C. Didierjean, R. Vanderesse and B. Jamart-Grégoire, Tetrahedron Lett., $2009,50,4158$.

9 Z. Zhou, C. Deng, C. Abbas, C. Didierjean, M.-C. Averlant-Petit, J. Bodiguel, R. Vanderesse and B. Jamart-Grégoire, Eur. J. Org. Chem., 2014, 7643.

10 Y. Zhang, R. M. Malamakal, D. M. Chenoweth, J. Am. Chem. Soc., 2015, 137, 12422.

11 F. Bizet, N. Tonali, J.-L. Soulier, A. Oliva, J. Kaffy, B. Crousse and S. Ongeri, N. J. Chem. 2018, 42, 17062.

12 L. Dufau, A. S. Marques Ressurreição, R. Fanelli, N. Kihal, A. Vidu, T.Milcent, J.-L. Soulier, J. Rodrigo, A. Desvergne, K. Leblanc, G. Bernadat, B. Crousse, M. Reboud-Ravaux and S. Ongeri, J. Med. Chem., 2012, 55, 6762.

13 M. A. Schmitt, S. H. Choi, I. A. Guzei and S. H. Gellman, J. Am. Chem. Soc. 2005, 127, 13130.

14 S. H. Choi, I. A. Guzei, L. C. Spencer, S. H. Gellman, J. Am. Chem. Soc. 2009, 131, 2917.

15 L. Guo, Y. Chi, Almeida, A. M.; Guzei, I. A.; Parker, B. K.; Gellman, S. H. J. Am. Chem. Soc. 2009, 131, 16018.

16 Guo, L.; Almeida, A. M.; Zhang, W.; Reidenbach, A. G.; Choi, S. H.; Guzei, I. A.; Gellman, S. H. J. Am. Chem. Soc. 2010, $132,7868$.

17 Y-H. Shin, D. E. Mortenson, K. A. Satyshur, K. T. Forest, S. H. Gellman, J. Am. Chem. Soc. 2013, 135, 8149.

18 L. Wang, P. Coric, K. Zhu, W-Q. Liu, M. Vidal, S. Bouaziz, S. Broussy, Org. Biomol. Chem. 2018, 16, 459.

19 M. Pelay-Gimeno, A. Glas, O. Koch and T. N. Grossmann, Angew. Chem. Int. Ed., 2015, 54, 8896.

20 J. Laxio-Arenas, J. Kaffy and S. Ongeri, Curr. Opin. Chem. Biol. 2019, 52, 157.

21 (a) X. Li, J. Taechalertpaisarn, D. Xin, K. Burgess Org. Lett., 2015, 17, 632; b) E. Ko, A. Raghuraman, L. M. Perez, T. R. loerger, K. Burgess J. Am. Chem. Soc. 2012, 135, 167.

22 S. Pellegrino, N. Tonali, E. Erba, J. Kaffy, M. Taverna, A. Contini, M. Taylor, D. Allsop, M. L. Gelmi, S. Ongeri, Chem. Sci., 2017, 8, 1295.

23 J. Li, J.-J. Koh, S. Liu, R. Lakshminarayanan, C. S. Verma and R. W.Beuerman, Front. Neurosci., 2017, 11, 73.

24 M. R. Felício, O. N. Silva, S. Gonçalves, N. C. Santos and O. L. Franco, Front. Chem., 2017, 5, 5.

25 D. Kalafatovic and E. Giralt, Molecules, 2017, 22, 1929.

26 C. Byrne, M. Belnou, E.-E. Baulieu, O. Lequin and Y. Jacquot, Pept. Sci., 2019, e24113.

27 E. Polak and G. Ribière, Revue Française d'Informatique et de Recherche Opérationnelle, 1969, $16,35$.

28 J. L. Banks, H. S. Beard, Y. Cao, A. E. Cho, W. Damm, R. Farid, A. K. Felts, T. A. Halgren, D. T. Mainz, J. R. Maple, R. Murphy, D. M. Philipp, M. P. Repasky, L. Y. Zhang, B. J. Berne, R. A. Friesner, E. Gallicchio and R. M. Levy, J. Comp. Chem., $2005,26,1752$.

29 W. C. Still, A. Tempczyk, R. C. Hawley and T. Hendrickson, J. Am. Chem. Soc. 1990, 112, 6127.

30 MacroModel, version 10.2, Schrodinger, LLC, New York, NY (USA) 2013.

31 G. Chang, W. C. Guida and W. C. Still, J. Am. Chem. Soc., 1989, 111, 4379.

32 (a) P. Hohenberg and W. Kohn, Phys. Rev., 1964, 136, B864-B871; (b) W. Kohn and L. J. Sham, Phys. Rev., 1965, 140, A1133.

33 (a) C. Lee, W. Yang and R. G. Parr, Phys. Rev. B, 1988, 37, 785-789; (b) A. D. Becke, J. Chem. Phys., 1993, 98, 5648.

34 R. A. Kendall, T. H. Dunning Jr. and R. J. Harrison, J. Chem. Phys., 1992, 96, 6796.

35 S. Grimme, J. Antony, S. Ehrlich and H. Krieg, J. Chem. Phys., 2010, 132, 154104.

36 J. Tomasi, B. Mennucci and R. Cammi, Chem. Rev., 2005, 105, 2999.

37 M. J. Frisch, G. W. Trucks, H. B. Schlegel, G. E. Scuseria, M. A. Robb, J. R. Cheeseman, G. Scalmani, V. Barone, G. A. Petersson, H. Nakatsuji, X. Li, M. Caricato, A. V. Marenich, J. Bloino, B. G. Janesko, R. Gomperts, B. Mennucci, H. P. Hratchian, J. V. Ortiz, A. F. Izmaylov, J. L. Sonnenberg, D. Williams-Young, F. Ding, F. Lipparini, F. Egidi, J. Goings, B. Peng, A. Petrone, T. Henderson, D. Ranasinghe, V. G. Zakrzewski, J. Gao, N. Rega, G. Zheng, W. Liang, M. Hada, M. Ehara, K. Toyota, R. Fukuda, J. Hasegawa, M. Ishida, T. Nakajima, Y. Honda, O. Kitao, H. Nakai, T. Vreven, K. Throssell, J. A. Montgomery, Jr., J. E. Peralta, F. Ogliaro, M. J. Bearpark, J. J. Heyd, E. N. Brothers, K. N. Kudin, V. N. Staroverov, T. A. Keith, R. Kobayashi, J. Normand, K. Raghavachari, A. P. Rendell, J. C. Burant, S. S. Iyengar, J. Tomasi, M. Cossi, J. M. Millam, M. Klene, C. Adamo, R. Cammi, J. W. Ochterski, R. L. Martin, K. Morokuma, O. Farkas, J. B. Foresman and D. J. Fox, Gaussian 16, Revision B.01, Gaussian, Inc., Wallingford CT (USA) 2016. 


\section{Introducing sequential aza-amino acids induces repeated $\beta$ - turns and helical conformations in peptides}

N. Tonali, ${ }^{[a]}$ I. Correia, ${ }^{[b]}$ J. Lesma,${ }^{[a]}$ G. Bernadat, ${ }^{[a]}$ S. Ongeri, ${ }^{*[a]}$, O. Lequin, ${ }^{[b]}$

[a] Université Paris Saclay, CNRS, BioCIS, 92296 Châtenay-Malabry Cedex, France.

E-mail: sandrine.ongeri@universite-paris-saclay.fr

[b] Sorbonne Université, Ecole Normale Supérieure, PSL University, CNRS, Laboratoire des Biomolécules, 4 place Jussieu, 75252 Paris Cedex 05, France.

Email: olivier.lequin@sorbonne-universite.fr

\section{Table of contents}

Pages S2-S11 Synthesis and characterization of Boc-Val-Ala-Val- $\mathrm{NH}_{2}$ 1, Boc-aVal-aAla-Val-OBn 3, and Boc-aVal-aAla-Val-aVal-aAla-Val- $\mathrm{NH}_{2} 5$

Pages S12-S27 NMR data of compounds 1-5: assignment tables, conformational parameters and NMR spectra

Page S28 Crystallographic data for Boc-aVal-aAla-Val- $\mathrm{NH}_{2} 2$

Pages S29-S31 NMR structures

Pages S32-S36 Ab initio conformational search: molecular mechanics and DFT calculations

Pages S37-S61 Atomic coordinates of molecular models refined by DFT 


\section{Synthesis and characterization of Boc-Val-Ala-Val- $\mathrm{NH}_{2}$ 1, Boc-aVal-aAla-Val-OBn 3, and Boc-aVal-aAla-Val-aVal-aAla-Val- $\mathrm{NH}_{2} 5$}

\section{General Experimental Methods}

Usual dry solvents were purchased from commercial sources. 4-nitrophenyl chloroformate, $N, N, N^{\prime}, N^{\prime}-$ Tetramethyl-O-(1H-benzotriazol-1-yl)uronium hexafluorophosphate (HBTU), 4-(4,6-dimethoxy-1,3,5-triazin-2yl)-4-methylmorpholinium $\quad$ chloride $\quad\left(\mathrm{DMTMM}\left(\mathrm{Cl}^{-}\right)\right), \quad$ 1-hydroxybenzotriazole $\quad(\mathrm{HOBt}), \quad 1-$ [Bis(dimethylamino)methylene]-1H-1,2,3-triazolo[4,5-b]pyridinium 3-oxid hexafluorophosphate (HATU), 1Hydroxy-7-azabenzotriazole (HOAt), Boc-NH-Val-OH, L-Ala-OCH $H_{3}, L-V a l-\mathrm{NH}_{2}$ and L-Val-OBn were purchased from commercial sources. Boc-aVal-aAla-Val-NH 2 and Boc-aGly-aGly-Val-NH 4 were prepared according to our published methods (F. Bizet, N. Tonali, J.-L. Soulier, A. Oliva, J. Kaffy, B. Crousse, S. Ongeri, N. J. Chem. 2018, 42, 17062-17072; L. Dufau, A. S. Marques Ressurreição, R. Fanelli, N. Kihal, A. Vidu, T.Milcent, J.-L. Soulier, J. Rodrigo, A. Desvergne, K. Leblanc, G. Bernadat, B. Crousse, M. ReboudRavaux,S. Ongeri, J. Med. Chem. 2012, 55, 6762-6775). Pure products were obtained after liquid chromatography using Merck silica gel 60 (40-63 $\mu \mathrm{m})$. TLC analyses were performed on silica gel 60F-250 (0.26 mm thickness) plates. The plates were visualized with UV light $(\lambda=254 \mathrm{~nm})$ or revealed with a $5 \%$ solution of phosphomolybdic acid in $\mathrm{EtOH}$ or with a solution of ninhydrin in $\mathrm{EtOH}$. Melting points were determined on a Kofler melting point apparatus. Element analyses $(\mathrm{C}, \mathrm{H}$, and $\mathrm{N})$ were performed on a PerkinElmer C, H, N Analyzer 2400 at the Microanalyses Service of the Faculty of Pharmacy at ChâtenayMalabry (BioCIS, France). NMR spectra were recorded on an Ultrafield Bruker AVANCE $300\left({ }^{1} \mathrm{H}, 300 \mathrm{MHz}\right.$, $\left.{ }^{13} \mathrm{C}, 75 \mathrm{MHz}\right)$ or on a Bruker Avance $400\left({ }^{1} \mathrm{H}, 400 \mathrm{MHz},{ }^{13} \mathrm{C}, 100 \mathrm{MHz}\right)$, or on a Bruker NMR spectrometer operating at a ${ }^{1} \mathrm{H}$ frequency of $500.3 \mathrm{MHz}$ and equipped with either a room temperature TXI probe or a cryogenic TCl probe. Chemical shifts $\delta$ are in ppm, and the following abbreviations are used: singlet (s), broad singlet (bs), doublet (d), doublet of triplet (dt), triplet (t), multiplet $(\mathrm{m})$. IR spectra were recorded on a Bruker Vector 22 FT-IR spectrometer. HRMS were obtained using a TOF LCT Premier apparatus (Waters), with an electrospray ionization source. The purity of compounds was determined by HPLC using the 2695 Alliance system (Waters) and a XBridge Select $\left(\mathrm{C}_{18}, 3.5 \mu \mathrm{m}, 150 \mathrm{~mm} \times 2.1 \mathrm{~mm}\right)$; mobile phase, $\mathrm{MeCN} / \mathrm{H}_{2} \mathrm{O}$ $+0.1 \%$ formic acid from 5 to $100 \%$ in $20 \mathrm{~min}$; detection at $220 \mathrm{~nm}$ or $245 \mathrm{~nm}$; flow rate $0.25 \mathrm{~mL} / \mathrm{min}$. 


$$
\text { (S) }
$$

Methyl (2S)-2-[[(2S)-2-(tert-butoxycarbonylamino)-3-methyl-butanoyl]amino]propanoate (6): Boc-NHVal-OH (500 mg, $2.3 \mathrm{mmol}, 1.0$ eq.) was dissolved in DMF $(10.0 \mathrm{~mL})$ under a nitrogen atmosphere and the solution was cooled at $0^{\circ} \mathrm{C}$. At this moment, $\mathrm{HOBt}(387 \mathrm{mg}, 2.5 \mathrm{mmol}, 1.1$ eq.) and HBTU (960 mg, 2.5 mmol, 1.1 eq.) were added. The solution was stirred at $0^{\circ} \mathrm{C}$ for $30 \mathrm{~min}$ and the solution of $\mathrm{HCl} \cdot \mathrm{NH}_{2}-\mathrm{Ala}-\mathrm{OCH}_{3}$ (321 mg, $2.3 \mathrm{mmol}, 1.0$ eq.) and DIPEA (1.2 mL, $6.9 \mathrm{mmol}, 3.0$ eq.) in DMF (10.0 mL) was added. The reaction was stirred at room temperature overnight. After evaporation of the volatiles under vacuum, the oily residue was taken up with EtOAc. The organic layer was successively washed with $10 \%$ aqueous citric solution, water, $10 \%$ aqueous $\mathrm{K}_{2} \mathrm{CO}_{3}$ solution, brine, and then dried over $\mathrm{Na}_{2} \mathrm{SO}_{4}$, filtered and concentrated under reduced pressure. Purification by column chromatography on silica gel using Cyclohexane/EtOAc 7:3 as eluent afforded compound $6(564 \mathrm{mg}, 1.87 \mathrm{mmol}, 81 \%)$ as a white solid. $\mathrm{R}_{f}=0.35$ (Cyclohexane /EtOAc 7:3); ${ }^{1} \mathrm{H}$ NMR (DMSO-d $\left.6,300 \mathrm{MHz}\right): 8.29(1 \mathrm{H}, \mathrm{d}, J=6.5 \mathrm{~Hz}) ; 6.61(1 \mathrm{H}, \mathrm{d}, J=9.0 \mathrm{~Hz}) ; 4.25(1 \mathrm{H}, \mathrm{m}) ; 3.78$ $(1 \mathrm{H}, \mathrm{m}) ; 3.60(3 \mathrm{H}, \mathrm{s}) ; 1.92(1 \mathrm{H}, \mathrm{m}) ; 1.37(9 \mathrm{H}, \mathrm{s}) ; 1.27(3 \mathrm{H}, \mathrm{d}, J=7.3 \mathrm{~Hz}) ; 0.86(3 \mathrm{H}, \mathrm{d}, J=6.7 \mathrm{~Hz}) ; 0.81(3 \mathrm{H}$, $\mathrm{d}, J=6.7 \mathrm{~Hz}$ ) ${ }^{13} \mathrm{C}$ NMR (DMSO-d 6 , $75 \mathrm{MHz}$ ): 172.9; 171.3; 155.4; 78.0; 59.1; 51.8; 47.5; 30.6; 28.2; 19.1, 18.0; 16.9; mp = 142-144 ${ }^{\circ} \mathrm{C}$; IR: $3310 \mathrm{~cm}^{-1} ; 2976 \mathrm{~cm}^{-1} ; 1751,1682,1649 \mathrm{~cm}^{-1} ; 1557,1524 \mathrm{~cm}^{-1} ; 1160 \mathrm{~cm}^{-1}$. HRMS (TOF ESI, ion polarity positive): $\mathrm{m} / \mathrm{z} 325.1739$ calc. for $\left[\mathrm{C}_{14} \mathrm{H}_{26} \mathrm{~N}_{2} \mathrm{O}_{5}+\mathrm{Na}\right]^{+}$, found 325.1746 .

\section{tert-butyl}

$\mathrm{N}-[(1 \mathrm{~S})-1-[[(1 S)-2-[[(1 S)-1-c a r b a m o y l-2-m e t h y l-p r o p y l] a m i n o]-1-m e t h y l-2-o x o-$ ethyl]carbamoyl]-2-methyl-propyl]carbamate (1): Compound 6 (495 mg, $1.64 \mathrm{mmol}, 1.0$ eq.) was dissolved in $\mathrm{MeOH}(20.0 \mathrm{~mL})$ and an aqueous solution of $\mathrm{NaOH} 2 \mathrm{M}(4.1 \mathrm{~mL}, 8.19 \mathrm{mmol}, 5.0$ eq.) was added dropwise to the solution. The reaction was stirred at $60^{\circ} \mathrm{C}$ for $1 \mathrm{~h}$. The volatiles were evaporated and the solid obtained was solubilized in water. The mixture was acidified with $10 \%$ aqueous $\mathrm{KHSO}_{4}$ solution until $\mathrm{pH}=2-3$. The product was extracted from the water phase with $\mathrm{DCM}$. The organic phase was dried over $\mathrm{Na}_{2} \mathrm{SO}_{4}$ and concentrated under vacuum to afford the free carboxylic acid (417 mg, $\left.1.45 \mathrm{mmol}, 89 \%\right)$ that was used without any further purification and dissolved in anhydrous DMF $(10 \mathrm{~mL})$. The solution was cooled at $0^{\circ} \mathrm{C}$ and HOBt $(245 \mathrm{mg}, 1.6 \mathrm{mmol}, 1.1 \mathrm{eq}$.$) and HBTU (607 \mathrm{mg}, 1.6 \mathrm{mmol}, 1.1$ eq. $)$ were added. The reaction mixture was stirred at $0^{\circ} \mathrm{C}$ for $30 \mathrm{~min}$ and the solution of $\mathrm{HCl} \cdot \mathrm{NH}_{2}-\mathrm{Val}^{-} \mathrm{NH}_{2}(221 \mathrm{mg}, 1.45 \mathrm{mmol}$, 1.0 eq.) and DIPEA (0.74 mL, $4.35 \mathrm{mmol}, 3.0$ eq.) in DMF $(10.0 \mathrm{~mL})$ were added. The reaction was carried at room temperature overnight. After evaporation of the volatiles under vacuum, the oily residue was taken up with EtOAc. After few minutes a white solid precipitated which after filtration afforded compound 1 (440 $\mathrm{mg}, 1.14 \mathrm{mmol}, 79 \%$ so $70 \%$ for two steps from compound 6 ) as a white solid. ${ }^{1} \mathrm{H}$ NMR (DMSO-d $6,400 \mathrm{MHz}$ ): $7.92(1 \mathrm{H}, \mathrm{d}, J=7.2 \mathrm{~Hz}) ; 7.69(1 \mathrm{H}, \mathrm{d}, J=8.9 \mathrm{~Hz}) ; 7.36(1 \mathrm{H}, \mathrm{s}) ; 7.01(1 \mathrm{H}, \mathrm{s}) ; 6.71(1 \mathrm{H}, \mathrm{d}, J=8.9 \mathrm{~Hz}) ; 4.38$ $(1 \mathrm{H}, \mathrm{m}) ; 4.09(1 \mathrm{H}, \mathrm{dd}, J=8.8,6.5 \mathrm{~Hz}) ; 3.80(1 \mathrm{H}, \mathrm{m}) ; 1.94(2 \mathrm{H}, \mathrm{m}) ; 1.37(9 \mathrm{H}, \mathrm{s}) ; 1.19(3 \mathrm{H}, \mathrm{d}, J=6.9 \mathrm{~Hz}) ; 0.82$ $(12 \mathrm{H}, \mathrm{m}) ;{ }^{13} \mathrm{C}$ NMR (DMSO-d 6 , $\left.400 \mathrm{MHz}\right): 172.7 ; 171.8 ; 170.9 ; 155.4 ; 78.0 ; 59.5 ; 57.2 ; 48.0 ; 30.5,30.3 ; 28.2$; 
19.2; 18.1; 17.9; 17.8;; mp = 266-270 ${ }^{\circ} \mathrm{C} ; \mathrm{IR}: 3293 \mathrm{~cm}^{-1} ; 2960 \mathrm{~cm}^{-1} ; 1671,1626 \mathrm{~cm}^{-1} ; 1529 \mathrm{~cm}^{-1}$. HRMS (TOF ESI, ion polarity positive): $\mathrm{m} / \mathrm{z} 387.2607$ calc. for $\left[\mathrm{C}_{18} \mathrm{H}_{35} \mathrm{~N}_{4} \mathrm{O}_{5}+\mathrm{H}\right]^{+}$and 409.2427 calc. for $\left[\mathrm{C}_{18} \mathrm{H}_{35} \mathrm{~N}_{4} \mathrm{O}_{5}+\mathrm{Na}\right]^{+}$, found 387.2602 and 409.2427; Elemental analysis: $\mathrm{C}_{18} \mathrm{H}_{34} \mathrm{~N}_{4} \mathrm{O}_{5}+1.5 \mathrm{H}_{2} \mathrm{O}$ calcd C 52.28, $\mathrm{H}$ 9.04, N 13.55; found C 52.37, H 8.24, N 13.60. HPLC purity (XBridge Select C18, $3.5 \mu \mathrm{m}, \mathrm{H}_{2} \mathrm{O}+0.1 \%$ form. ac./ACN - grd 5-100\% in $20 \mathrm{~min}$, detection at $245 \mathrm{~nm}$ ): TR $=11.83 \mathrm{~min}, 100 \%$.<smiles>[3H]C(C)N(NC(=O)OC(C)(C)C)C(=O)NNC</smiles>

1- 4-nitrophenyl chloroformate, DIPEA, DMF

2- DMAP $66 \%$<smiles>CCCOC(=O)[C@H](NC(=O)N(C)NC(=O)N(NC(=O)OC(C)(C)C)C(C)C)C(C)C</smiles>

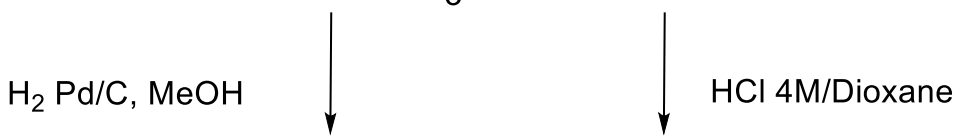

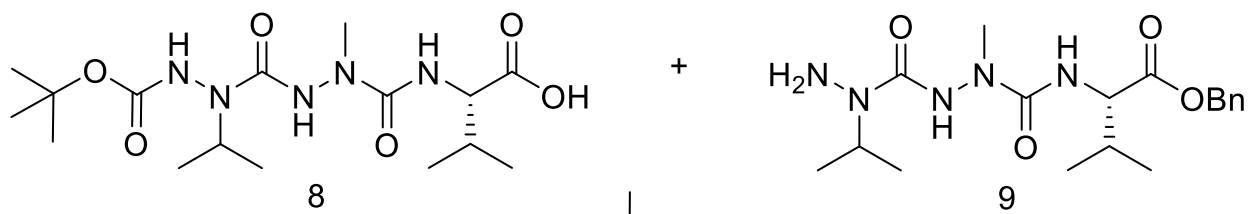
HATU, HOAT, DIPEA, DMF $16 \%$<smiles>CC(C)C(NC(=O)N(C)NC(=O)N(NC(=O)OC(C)(C)C)C(C)C)C(=O)NN(C(=O)NN(C)C(=O)N[C@H](C(=O)OCc1ccccc1)C(C)C)C(C)C</smiles>

1- $\mathrm{H}_{2} \mathrm{Pd} / \mathrm{C}, \mathrm{MeOH}$

2- DMTMM, $\mathrm{NH}_{3} / \mathrm{MeOH}$, $\mathrm{IPrOH}$ $33 \%$<smiles>CC(C)[C@H](NC(=O)N(C)NC(=O)N(NC(=O)N(C)C(C)C)C(=O)N[C@@H](C(N)=O)C(C)C)C(=O)NN(C)C(=O)NN(C(=O)OC(C)(C)C)C(C)C</smiles>

\section{Scheme S2}


Benzyl (2S)-2-[[[[(tert-butoxycarbonylamino)-isopropyl-carbamoyl]amino]-methyl-carbamoyl]amino]3-methyl-butanoate (3): To a solution of H-L-Val-OBn hydrochloride $(110 \mathrm{mg}, 0.45 \mathrm{mmol}$; $1.1 \mathrm{eq}$.) in dry DMF $(5 \mathrm{~mL})$, were added under argon atmosphere DIPEA $(0.16 \mathrm{~mL}, 0.90 \mathrm{mmol}, 2.2$ eq.) and p-nitrophenyl chloroformate $(90.7 \mathrm{mg}, 0.45 \mathrm{mmol}, 1.1 \mathrm{eq}$.). The clear solution obtained was stirred at room temperature and monitored by TLC. After formation of the activated intermediate, a solution of compound 7 (100 mg, 0.41 mmol) and DMAP (20.1 mg, $0.41 \mathrm{mmol}, 1.0$ eq. $)$ in DMF $(1 \mathrm{~mL})$ was added to the reaction mixture and the reaction was stirred overnight at room temperature. After evaporation of the volatiles under vacuum, the oily residue was purified by chromatography on silica gel using EtOAc 100\% to afford compound 3 (130 mg, 0.27 mmol, $66 \%)$ as a white solid. $\mathrm{R}_{f}=0.55($ EtOAc $100 \%){ }^{1} \mathrm{H}$ NMR $\left(400 \mathrm{MHz}, \mathrm{DMSO}-\mathrm{d}_{6}\right): \delta=7.33(5 \mathrm{H}, \mathrm{m}) ; 7.18$ $(1 \mathrm{H}, \mathrm{bp}) ; 6.35(1 \mathrm{H}, \mathrm{bp}) ; 6.19(1 \mathrm{H}, \mathrm{bp}) ; 5.16(2 \mathrm{H}, \mathrm{m}) ; 4.66(1 \mathrm{H}, \mathrm{m}) ; 4.42(1 \mathrm{H}, \mathrm{m}) ; 3.14(3 \mathrm{H}, \mathrm{s}) ; 2.09(1 \mathrm{H}, \mathrm{m})$; $1.49(9 \mathrm{H}, \mathrm{s}) ; 1.12(6 \mathrm{H}, \mathrm{m}) .0 .93(6 \mathrm{H}, \mathrm{m}) ;{ }^{13} \mathrm{C}$ NMR ( $\left.100 \mathrm{MHz}, \mathrm{DMSO}-\mathrm{d}_{6}\right): 173.0 ; 158.6 ; 151.9 ; 144.5 ; 135.9$; 128.6, 128.3; 82.9; 66.5; 58.7; 48.9; 36.2; 31.1; 28.1; 19.2; 18.9; $\mathrm{mp}=174-176{ }^{\circ} \mathrm{C} ; \mathrm{IR}: 3391,3296 \mathrm{~cm}^{-1}(\mathrm{~N}-$ $\mathrm{H}$ stretch); $2975 \mathrm{~cm}^{-1}$ (C-H stretch); 1740, 1725, 1673, $1643 \mathrm{~cm}^{-1}$ (C=O stretch); 1536, $1494 \mathrm{~cm}^{-1}$ (N-H bend); 1272, $1243 \mathrm{~cm}^{-1}$ (C-N stretch); $1179 \mathrm{~cm}^{-1}$ (C-C(O)-C stretch); HRMS (TOF ESI, ion polarity positive): $\mathrm{m} / \mathrm{z}$ 480.2822 calc. for $\left[\mathrm{C}_{23} \mathrm{H}_{38} \mathrm{~N}_{5} \mathrm{O}_{6}+\mathrm{H}\right]^{+}$, found 480.2823; HPLC purity (XBridge Select C18, $3.5 \mu \mathrm{m}, \mathrm{H}_{2} \mathrm{O}+0.1 \%$ form. ac. $/ A C N-$ grd $5-100 \%$ in $20 \mathrm{~min}$ ): TR $=17.63 \mathrm{~min}, 97 \%$

(2S)-2-[[[[(tert-butoxycarbonylamino)-isopropyl-carbamoyl]amino]-methyl-carbamoyl]amino]-3methyl-butanoic acid (8): 3 (100 mg, $0.21 \mathrm{mmol}, 1.0 \mathrm{eq})$ was dissolved in methanol $(3 \mathrm{~mL})$ and then $\mathrm{Pd} / \mathrm{C}$ $10 \%$ was added $(10 \mathrm{mg})$. The reaction mixture was stirred at room temperature under hydrogen atmosphere. After complete disappearance of the starting material, the mixture was filtered through a celite pad and after evaporation of the volatiles the desired product 8 was obtained $(82 \mathrm{mg}, 0.21 \mathrm{mmol}, 100 \%)$ and used without any further purification. HRMS (TOF ESI, ion polarity positive): $\mathrm{m} / \mathrm{z} 390.2308$ calc. for $\left[\mathrm{C}_{16} \mathrm{H}_{31} \mathrm{~N}_{5} \mathrm{O}_{6}+\mathrm{H}\right]^{+}$and 412.2172 calc. for $\left[\mathrm{C}_{16} \mathrm{H}_{31} \mathrm{~N}_{5} \mathrm{O}_{6}+\mathrm{Na}\right]^{+}$, found 390.2345 and 412.2177 .

\title{
Benzyl (2S)-2-[[[[amino(isopropyl)carbamoyl]amino]-methyl-carbamoyl]amino]-3-methyl-butanoate
} (9):

To a solution of $3(100.0 \mathrm{mg}, 0.21 \mathrm{mmol}, 1.0 \mathrm{eq})$ in dioxane $(3 \mathrm{~mL})$, a $4 \mathrm{~N}$ solution of $\mathrm{HCl}$ in dioxane $(1.7 \mathrm{~mL}$, 40.0 eq.) was added at $0^{\circ} \mathrm{C}$. The mixture was stirred at room temperature until complete disappearance of the starting material (monitored by TLC). The desired product $9(79.7 \mathrm{mg}, 0.21 \mathrm{mmol}, 100 \%)$ was isolated as its hydrochloride salt by precipitation in diethyl ether and used for the next step without any further purification. HRMS (TOF ESI, ion polarity positive): $\mathrm{m} / \mathrm{z} 380.2253$ calc. for $\left[\mathrm{C}_{17} \mathrm{H}_{28} \mathrm{~N}_{4} \mathrm{O}_{6}+\mathrm{H}\right]^{+}$and 402.2117 calc. for $\left[\mathrm{C}_{17} \mathrm{H}_{28} \mathrm{~N}_{4} \mathrm{O}_{6}+\mathrm{Na}\right]^{+}$, found 380.2292 and 402.2139 .

\begin{abstract}
Benzyl (2S)-2-[[[[[[(2S)-2-[[[[(tert-butoxycarbonylamino)-isopropyl-carbamoyl]amino]-methylcarbamoyl]amino]-3-methyl-butanoyl]amino]-isopropyl-carbamoyl]amino]-methyl-carbamoyl]amino]3-methyl-butanoate (10): $8(79.7 \mathrm{mg}, 0.21 \mathrm{mmol})$ was dissolved in DMF (2 mL) under hydrogen atmosphere and cooled at $0^{\circ} \mathrm{C}$. At these time HOAt $(32 \mathrm{mg}, 0.23 \mathrm{mmol}, 1.1 \mathrm{eq})$ and HATU (88mg, 0.23 mmol, $1.1 \mathrm{eq})$ were added and the mixture was stirred for 1 hour. Then $9(81.8 \mathrm{mg}, 0.21 \mathrm{mmol})$ and DIPEA $(146 \mu \mathrm{L}, 0.84 \mathrm{mmol}, 4.0 \mathrm{eq})$ were added and the mixture was stirred at room temperature overnight. The volatiles were evaporated under reduced pressure, the residue was taken up with AcOEt and washed with citric acid $\left(10 \%\right.$ in water), saturated $\mathrm{NaHCO}_{3}$ and brine, dried over $\mathrm{Na}_{2} \mathrm{SO}_{4}$ filtered and concentrated under vacuum. The crude was purified by column chromatography on a silica gel using AcOEt/MeOH 95:5 as eluent to afford $10(24 \mathrm{mg}, 0.031 \mathrm{mmol}, 16 \%) \mathrm{R}_{f}=0.2(\mathrm{AcOEt} / \mathrm{MeOH} 95 / 5){ }^{1} \mathrm{H} \mathrm{NMR}\left(300 \mathrm{MHz}, \mathrm{CDCl}_{3}\right): \delta=$ $8.78(1 \mathrm{H}, \mathrm{bs}) ; 8.18(1 \mathrm{H}, \mathrm{bs}) ; 8.11(1 \mathrm{H}, \mathrm{bs}) ; 7.96(1 \mathrm{H}, \mathrm{bs}) ; 7.28(5 \mathrm{H}, \mathrm{m}) ; 6.61(1 \mathrm{H}, \mathrm{bs}) ; 6.10(1 \mathrm{H}, \mathrm{bs}) ; 5.14$ $(2 \mathrm{H}, \mathrm{m}) ; 4.62(2 \mathrm{H}, \mathrm{m}) ; 4.37(2 \mathrm{H}, \mathrm{m}) ; 3.20(6 \mathrm{H}, \mathrm{s}) ; 2.90(2 \mathrm{H}, \mathrm{m}) ; 1.39(9 \mathrm{H}, \mathrm{s}) ; 1.20-0.90(24 \mathrm{H}, \mathrm{m}) ;{ }^{13} \mathrm{C}$ NMR $\left(75 \mathrm{MHz}, \mathrm{CDCl}_{3}\right): 174.0 ; 157.5 ; 152.1 ; 142.1 ; 136.0 ; 128.7,127.0 ; 82.9 ; 66.5 ; 59.1 ; 48.9 ; 48.8 ; 36.4 ; 31.1$; 30.9; 28.3; 20.0; 19.6; 18.2; HRMS (TOF ESI, ion polarity positive): $\mathrm{m} / \mathrm{z} 751.4466$ calc. for $\left[\mathrm{C}_{34} \mathrm{H}_{58} \mathrm{~N}_{10} \mathrm{O}_{9}+\mathrm{H}\right]^{+}$ and 773.4286 calc. for $\left[\mathrm{C}_{34} \mathrm{H}_{58} \mathrm{~N}_{10} \mathrm{O}_{9}+\mathrm{Na}\right]^{+}$, found 751.4482 and 773.4291 ;
\end{abstract}

tert-butyl N-[[[(1S)-1-[[[[(1S)-1-carbamoyl-2-methyl-propyl]carbamoyl-methyl-amino]carbamoylisopropyl-amino]carbamoyl]-2-methyl-propyl]carbamoyl-methyl-amino]carbamoyl-isopropyl-

amino]carbamate (5): To a solution of $10(24.0 \mathrm{mg}, 0.031 \mathrm{mmol}, 1.0 \mathrm{eq})$ in methanol $(1 \mathrm{~mL})$ was added $\mathrm{Pd} / \mathrm{C} 10 \%(10.0 \mathrm{mg}, 0.009 \mathrm{mmol}, 0.3 \mathrm{eq})$. The reaction mixture was stirred at room temperature under hydrogen atmosphere. After complete disappearance of the starting material, the mixture was filtered through a celite pad. After evaporation of the volatiles under reduced pressure the white solid was dissolved in isopropanol $(1 \mathrm{~mL})$ and DMTMM. $\mathrm{HCl}(12.5 \mathrm{mg}, 0.045 \mathrm{mmol}, 1.5 \mathrm{eq})$ was added. At the resulting suspension, a solution of $\mathrm{NH}_{3}$ in methanol $(13 \mu \mathrm{L}, 0.087 \mathrm{mmol}, 3 \mathrm{eq})$ was slowly dropped. The mixture was stirred at room temperature for $3 \mathrm{~h}$. The volatiles were removed under vacuum, the residue was taken with 
AcOEt and the organic layer was washed with saturated aqueous $\mathrm{NaHCO}_{3}$, dried over $\mathrm{Na}_{2} \mathrm{SO}_{4}$, filtered and concentrated under vacuum. Purification by column chromatography on silica gel AcOEt/MeOH 95/5 and then $80 / 20$ afforded compound $5(6.3 \mathrm{mg}, 9.6 \mu \mathrm{mol}, 33 \%)$ as a white solid. $\mathrm{R}_{f}=0.1$ (AcOEt/MeOH 95/5). For ${ }^{1} \mathrm{H}$ and ${ }^{13} \mathrm{C}$ NMR, see Tables S13-S14. HRMS (TOF ESI, ion polarity positive): $\mathrm{m} / \mathrm{z} 660.4157$ calc. for $\left[\mathrm{C}_{27} \mathrm{H}_{53} \mathrm{~N}_{11} \mathrm{O}_{8}+\mathrm{H}\right]^{+}$and 682.3976 calc. for $\left[\mathrm{C}_{27} \mathrm{H}_{53} \mathrm{~N}_{11} \mathrm{O}_{8}+\mathrm{Na}\right]^{+}$found 660.4157 and 682.3978; HPLC purity (XBridge Select $\mathrm{C} 18,3.5 \mu \mathrm{m}, \mathrm{H}_{2} \mathrm{O}+0.1 \%$ form. ac./ACN - grd 5-100\% in $20 \mathrm{~min}$ ): TR $=15.12 \mathrm{~min}$.

\section{HRMS and HPLC-MS spectra of compound 1}

\section{Elemental Composition Report}

Multiple Mass Analysis: 2 mass(es) processed

Tolerance $=5.0$ PPM / DBE: $\min =-2.0, \max =100.0$

Selected filters: None

Monoisotopic Mass, Even Electron lons

580 formula(e) evaluated with 3 results within limits (up to 3 closest results for each mass)

Elements Used:

$\begin{array}{llll}\text { C: } 0-70 & H: 1-130 & N: 0-4 & \text { O: } 0-5 \quad \mathrm{Na}: 0-1\end{array}$

Minimum: $\quad 77.0 \quad-2.0$

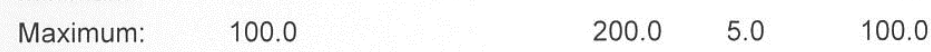

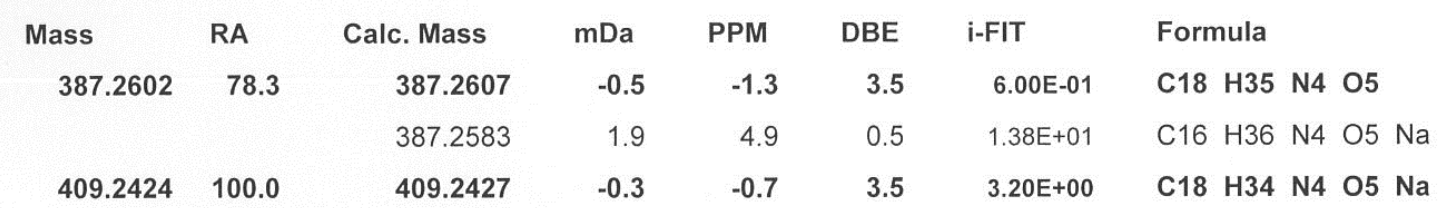

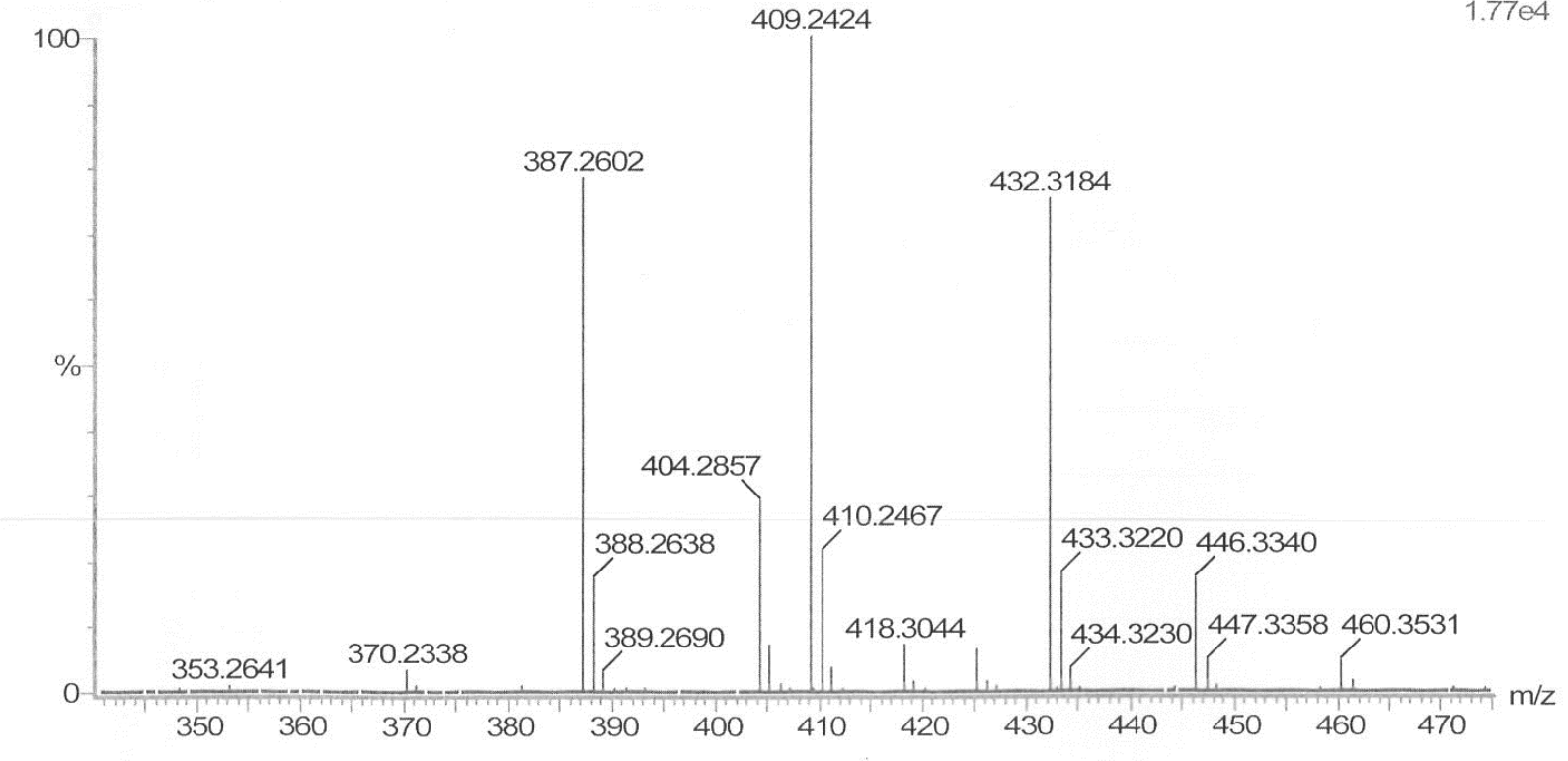




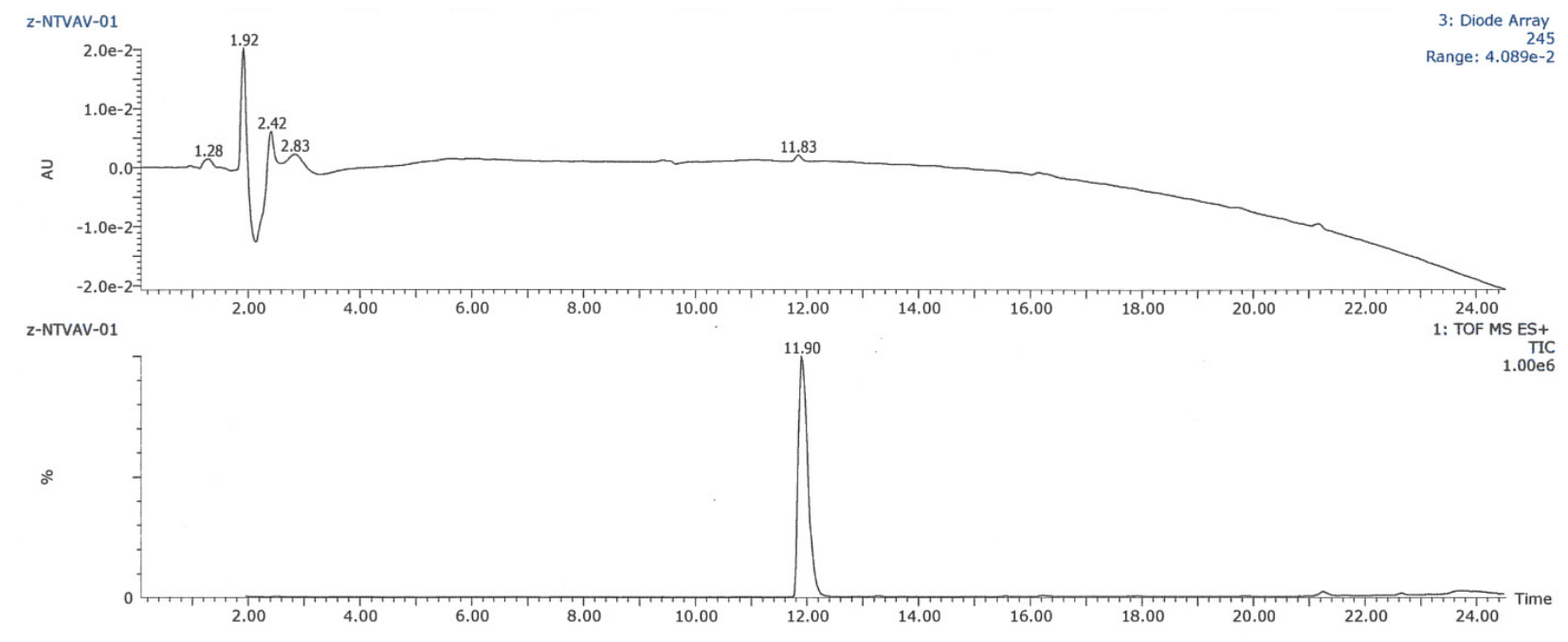

24-Nov-2015

Nicolo TONALI - MOLEC FLUOREES

z-NTVAV-01 354 (11.899) Cm (352:360-(336:346+375:386))

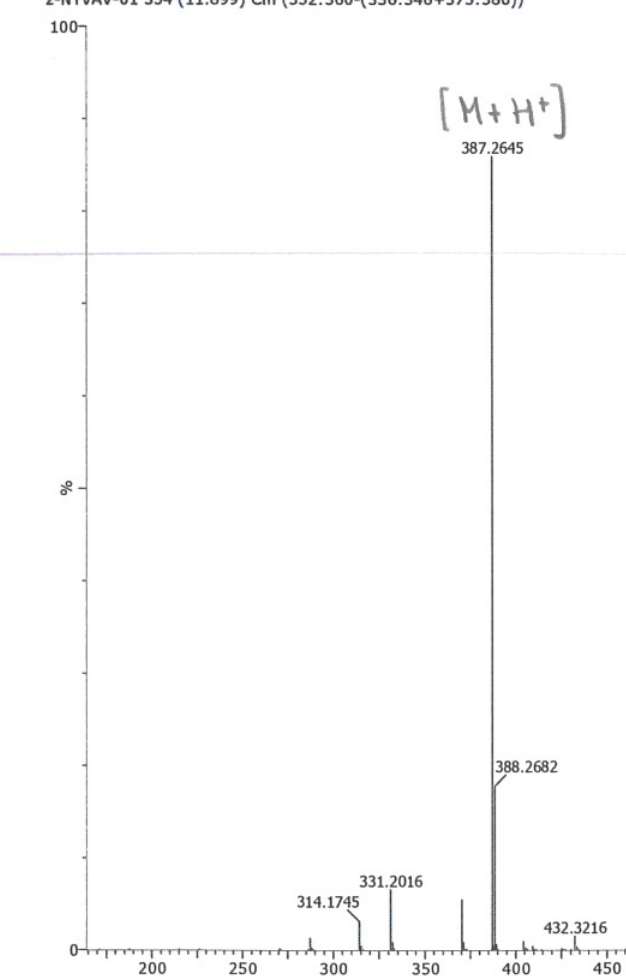

NT Val Ala Val

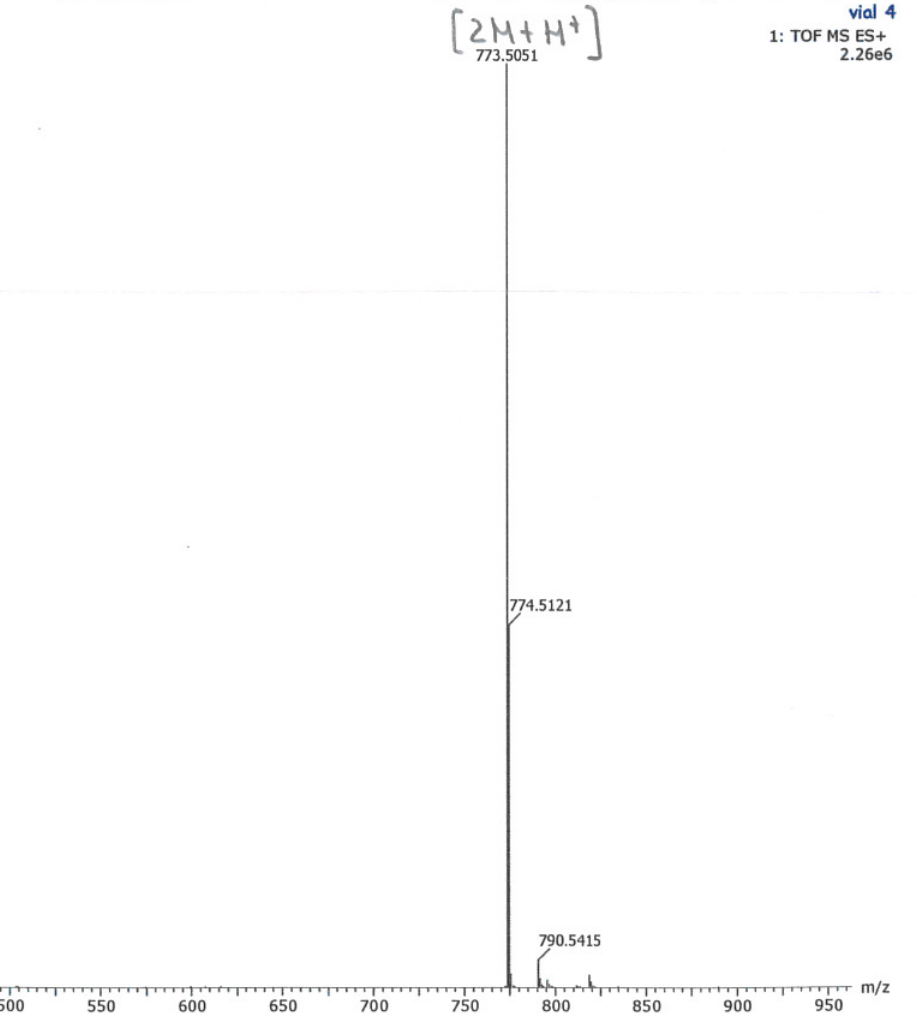




\section{HRMS and HPLC-MS spectra of compound 3}

\section{Elemental Composition Report}

Multiple Mass Analysis: 4 mass(es) processed

Tolerance $=5.0$ PPM / DBE: $\min =-2.0, \max =100.0$

Selected filters: None

Monoisotopic Mass, Even Electron lons

2266 formula(e) evaluated with 9 results within limits (up to 3 best isotopic matches for each mass)

Elements Used:

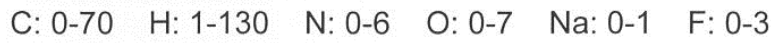

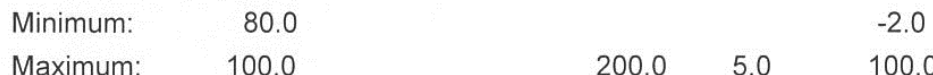

\begin{tabular}{|c|c|c|c|c|c|c|c|c|c|c|}
\hline Mass & RA & Calc. Mass & $\mathrm{mDa}$ & PPM & DBE & i-FIT & Formula & & & \\
\hline 480.2823 & 100.0 & 480.2822 & 0.1 & 0.2 & 7.5 & $1.20 \mathrm{E}+01$ & C23 H38 & N5 & 06 & \\
\hline & & 480.2814 & 0.9 & 1.9 & 5.5 & $1.48 E+01$ & $\mathrm{C} 24 \mathrm{H} 38$ & N3 & $\mathrm{O} 2$ & $\mathrm{NaF}$ \\
\hline & & 480.2834 & -1.1 & -2.3 & 3.5 & $1.50 \mathrm{E}+01$ & C2O H39 & N5 & 07 & $\mathrm{~F}$ \\
\hline
\end{tabular}

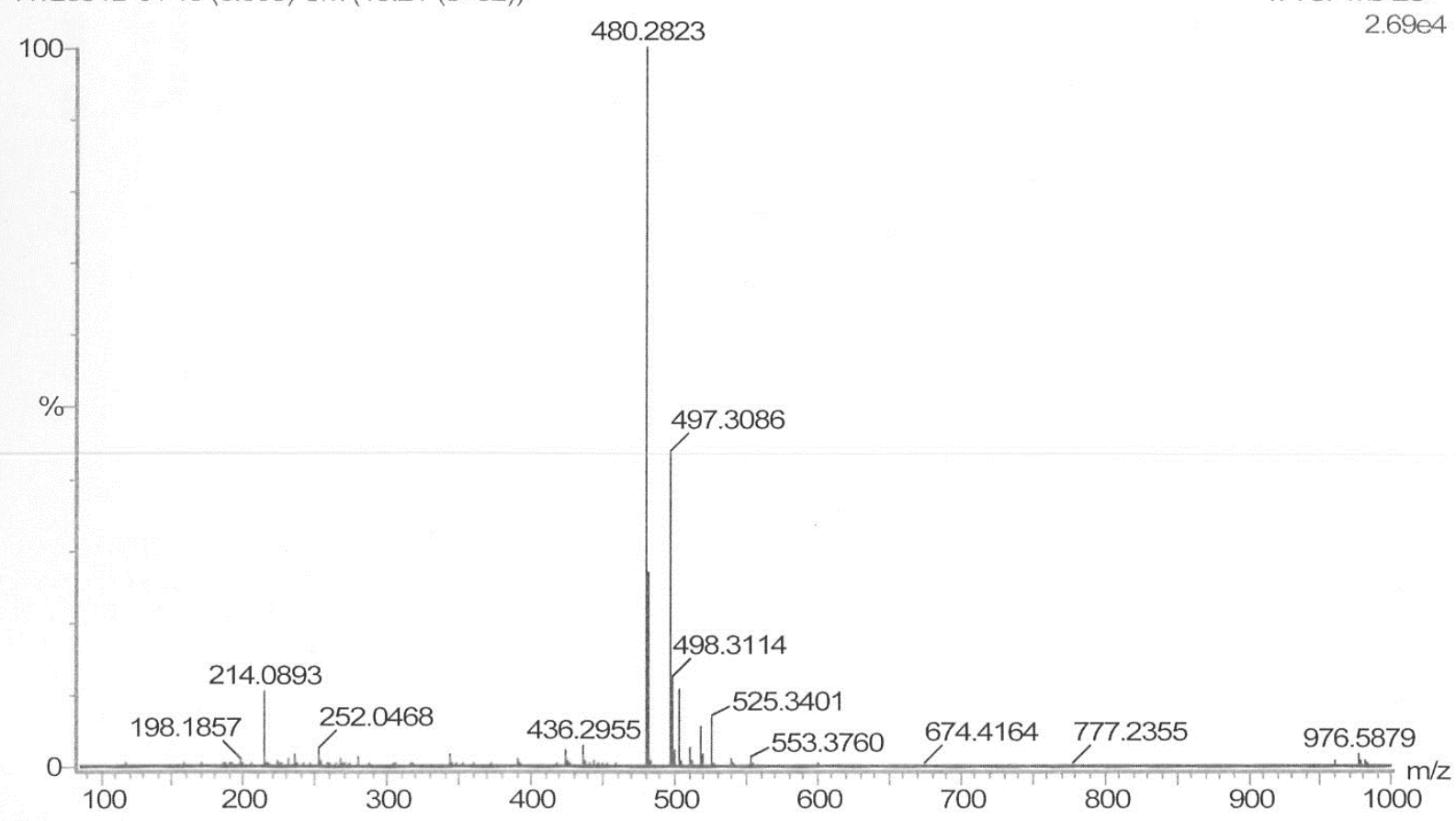


10-Nov-2015

Nicolo TONALI - MOLEC FLUOREES

NT $255 \mathrm{Val} \mathrm{Bn}$

Z-NT255VB-0

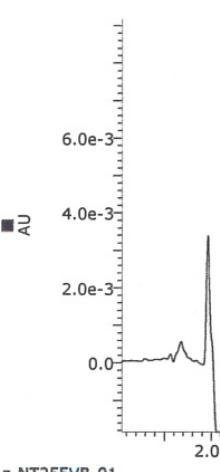

Z-NT255VB-01

80

$80-$

○

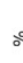

\section{0-Nov-2015}

Nicolo TONALI - MOLEC FLUOREES

z-NT255VB-01 559 (17.685) Cm (559:569-(517:536+603:619))

$$
{ }^{100} 7
$$

1007

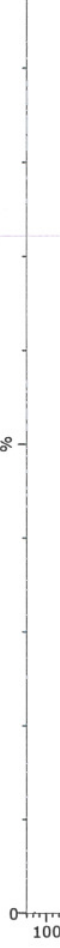

NT 255 Val Bn

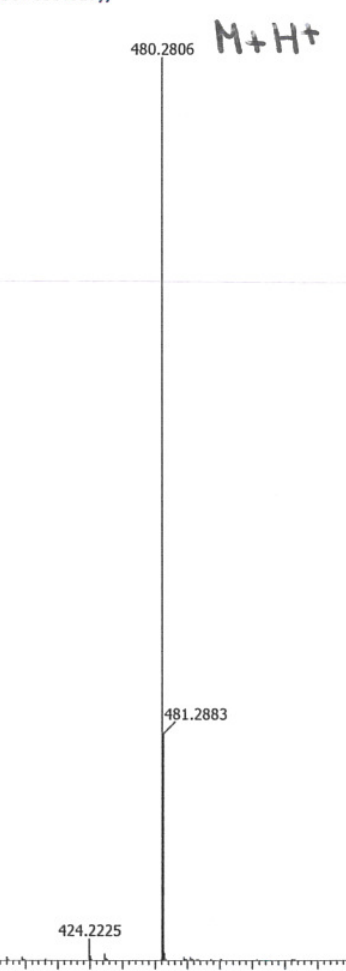

$\mathrm{H} 2 \mathrm{O}+0.1 \%$ ac. form. $/ \mathrm{ACN}-$ grd $5-100 \%$ en $20 \mathrm{~min}$

vial 3

3: Diode Array

Range: $1.189 \mathrm{e}-2$

$\begin{array}{rrrr}\text { Time } & \text { Height } & \text { Area } & \text { Area\% } \\ 17.63 & 8511 & 1523.04 & 97.20\end{array}$

$\begin{array}{rrrr}17.63 & 8511 & 1523.04 & 97.20\end{array}$

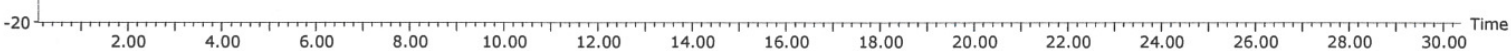

MS ES+
TIC
$1.43 \mathrm{e} 6$

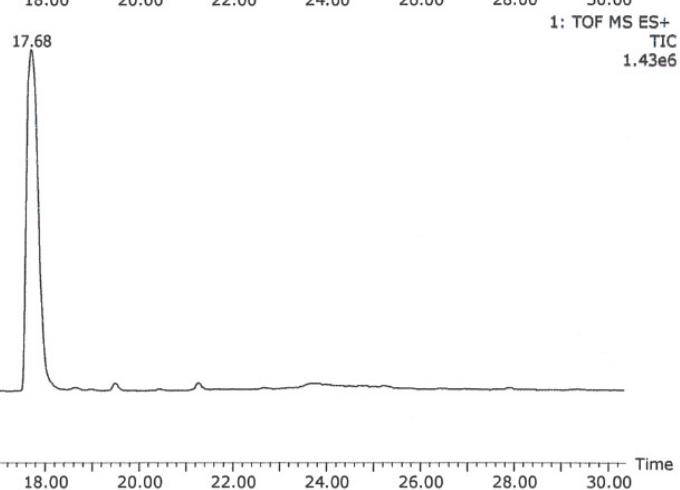

vial 3
1: TOF MSES+

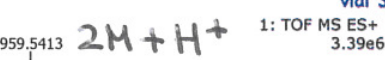

960.5574

915.5704

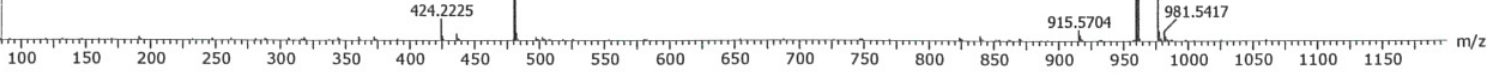




\section{HRMS and HPLC-MS spectra of compound 5}

\section{Elemental Composition Report}

Multiple Mass Analysis: 3 mass(es) processed

Tolerance $=5.0 \mathrm{PPM} / \mathrm{DBE}: \min =-2.0, \max =100.0$

Selected filters: None

Monoisotopic Mass, Even Electron lons

4804 formula(e) evaluated with 23 results within limits (up to 3 best isotopic matches for each mass)

Elements Used:

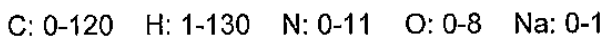

\begin{tabular}{|c|c|c|c|c|c|c|c|}
\hline Minimum: & 25.0 & & & & -2.0 & & \\
\hline Maximum: & 100.0 & & 200.0 & 5.0 & 100.0 & & \\
\hline Mass & RA & Calc. Mass & mDa & PPM & DBE & i-FIT & Formula \\
\hline \multirow[t]{3}{*}{660.4157} & 100 & 660.4157 & 0.0 & 0.0 & 6.5 & $4.53 \mathrm{E}+04$ & C27 H54 N11 O8 \\
\hline & & 660.4173 & -1.6 & -2.4 & 7.5 & $5.14 \mathrm{E}+04$ & C30 H55 N9 O6 $\mathrm{Na}$ \\
\hline & & 660.4133 & 2.4 & 3.6 & 3.5 & $3.65 E+04$ & $\mathrm{C} 25 \mathrm{H} 55 \mathrm{~N} 11 \mathrm{O} 8 \mathrm{Na}$ \\
\hline \multirow[t]{3}{*}{682.3978} & 29 & 682.3976 & 0.2 & 0.3 & 6.5 & $1.48 E+04$ & C27 H53 N11 O8 Na \\
\hline & & 682.4000 & -2.2 & -3.2 & 9.5 & $1.78 E+04$ & C29 H52 N11 O8 \\
\hline & & 682.3944 & 3.4 & 5.0 & 14.5 & $2.83 E+04$ & $\mathrm{C} 38 \mathrm{H} 53 \mathrm{~N} 5 \mathrm{O} 5 \mathrm{Na}$ \\
\hline
\end{tabular}

15-Feb-2018

JL 54

$\mathrm{H} 2 \mathrm{O} / \mathrm{MeOH}$ vial 27

Jacopo LESMA - MOLEC FLUOR

1: TOF MS ES+

JL54-01 $13(0.603) \mathrm{Cm}(12: 17-(3: 7+29: 32))$

$8.24 e 5$

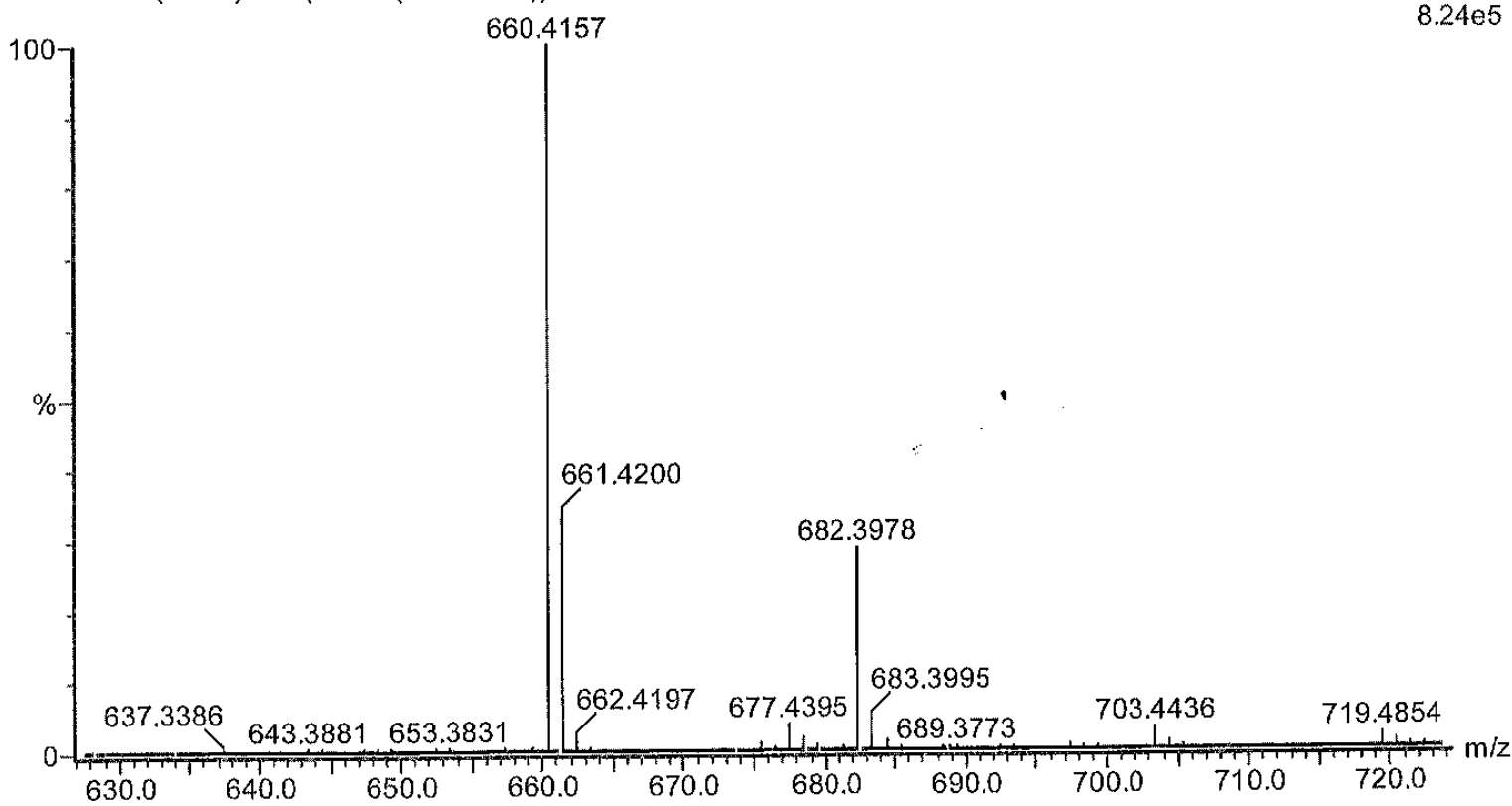




\section{Service D'Analyses HPLC-Masse}

\section{Or Biocis}

JL 54 F

22-Feb-2018

$z-J \operatorname{LS} 5 \mathrm{~F}-0$

-2.
LC-MS

JL $54 \mathrm{~F}$
HPLC Alliance 2695

MS-TOF - LCT Premier (Waters

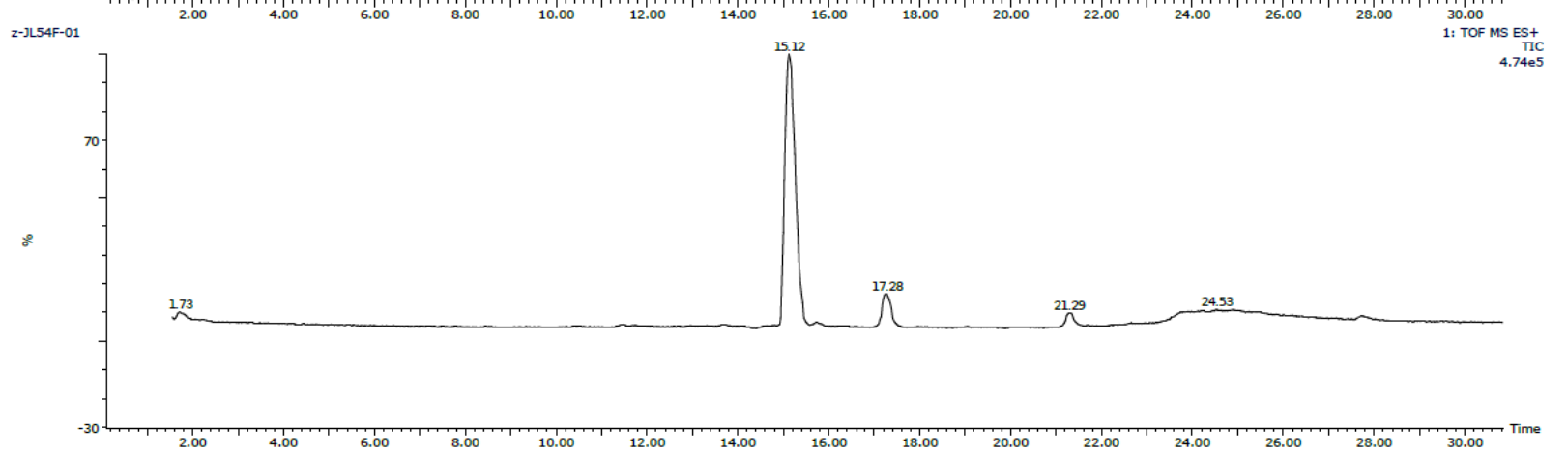

z- $-3: 54$ F- 91483 (15.122) $\mathrm{cm}(476: 495-(454: 468+501: 518))$

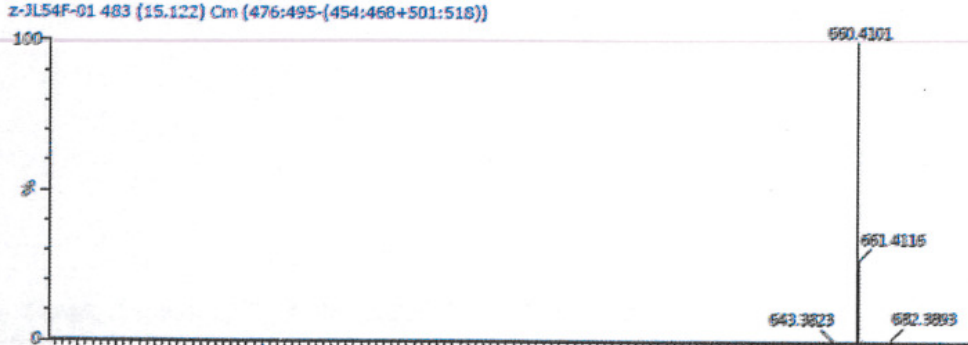




\section{NMR data of compounds 1-5}

\section{NMR data for peptide 1 (Boc-Val-Ala-Val-NH ${ }_{2}$ )}

Table S1. ${ }^{1} \mathrm{H}$ NMR chemical shifts of peptide $1(4 \mathrm{mM})$ in $\mathrm{CD}_{3} \mathrm{OH}$ at $293 \mathrm{~K}$

\begin{tabular}{ccccc}
\hline Residue & $\delta \mathrm{HN}(\mathrm{ppm}),{ }^{3} \mathrm{~J}(\mathrm{~Hz})$ & $\delta \mathrm{H}^{\mathrm{a}}(\mathrm{ppm}),{ }^{3} \mathrm{~J}(\mathrm{~Hz})$ & $\delta \mathrm{H}^{\mathrm{s}}(\mathrm{ppm})$ & $\delta$ Other protons $(\mathrm{ppm})$ \\
\hline $\mathrm{Boc}$ & & & & $\mathrm{CH}_{3} 1.44$ \\
$\mathrm{Val}^{1}$ & $6.62(\mathrm{~d}, J=8.7)$ & $3.87(\mathrm{dd}, J=8.7,7.0)$ & 2.03 & $\gamma \mathrm{CH}_{3} 0.95, \gamma^{\prime} \mathrm{CH}_{3} 0.91$ \\
$\mathrm{Ala}^{2}$ & $8.18(\mathrm{~d}, J=6.6)$ & $4.41(\mathrm{q}, J=6.9)$ & 1.36 & \\
$\mathrm{Val}^{3}$ & $7.86(\mathrm{~d}, J=8.6)$ & $4.17(\mathrm{dd}, J=8.6,6.8)$ & 2.07 & $\gamma \mathrm{CH}_{3} 0.96, \gamma^{\prime} \mathrm{CH}_{3} 0.95$ \\
$\mathrm{NH}_{2 \mathrm{Z}, \mathrm{E}}$ & $7.04(\mathrm{~s}), 7.60(\mathrm{~s})$ & & & \\
\hline
\end{tabular}

Table S2. ${ }^{13} \mathrm{C}$ NMR chemical shifts of peptide $1(4 \mathrm{mM})$ in $\mathrm{CD}_{3} \mathrm{OH}$ at $293 \mathrm{~K}$

\begin{tabular}{ccccc}
\hline Residue & $\delta \mathrm{CO}(\mathrm{ppm})$ & $\delta \mathrm{C}^{\alpha}(\mathrm{ppm})$ & $\delta \mathrm{C}^{\beta}(\mathrm{ppm})$ & $\delta$ Other carbons $(\mathrm{ppm})$ \\
\hline $\mathrm{Boc}$ & 158.0 & & & $\mathrm{Cq} 80.5, \mathrm{CH}_{3} 28.6$ \\
$\mathrm{Val}^{1}$ & 174.3 & 61.4 & 32.0 & $\gamma \mathrm{CH}_{3} 19.74, \gamma^{\prime} \mathrm{CH}_{3} 18.2$ \\
$\mathrm{Ala}^{2}$ & 174.7 & 50.4 & 17.9 & \\
$\mathrm{Val}^{3}$ & 176.1 & 59.8 & 31.8 & $\gamma \mathrm{CH}_{3} 19.69, \gamma^{\prime} \mathrm{CH}_{3} 18.3$ \\
\hline
\end{tabular}

Table S3. NMR conformational parameters for peptide 1 in $\mathrm{CD}_{3} \mathrm{OH}$ at $293 \mathrm{~K}$

\begin{tabular}{cccc}
\hline Residue & $\Delta \delta \mathrm{HN} / \Delta \mathrm{T}(\mathrm{ppb} / \mathrm{K})$ & ${ }^{3} \mathrm{JHN}-\mathrm{H} \alpha(\mathrm{Hz})$ & ${ }^{3} \mathrm{JH} \alpha-\mathrm{H} \beta(\mathrm{Hz})$ \\
\hline $\mathrm{Val}^{1}$ & -8.5 & 8.7 & 7.0 \\
$\mathrm{Ala}^{2}$ & -7.7 & 6.6 & 6.9 \\
$\mathrm{Val}^{3}$ & -8.4 & 8.6 & 6.8 \\
$\mathrm{NH}_{2 \mathrm{Z}, \mathrm{E}}$ & $-7.1,-8.3$ & & \\
\hline
\end{tabular}




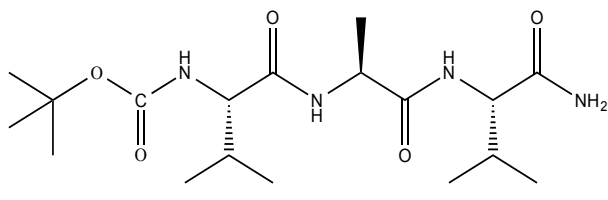

Boc $\quad$ V1 $\quad$ A2 $\quad$ V3
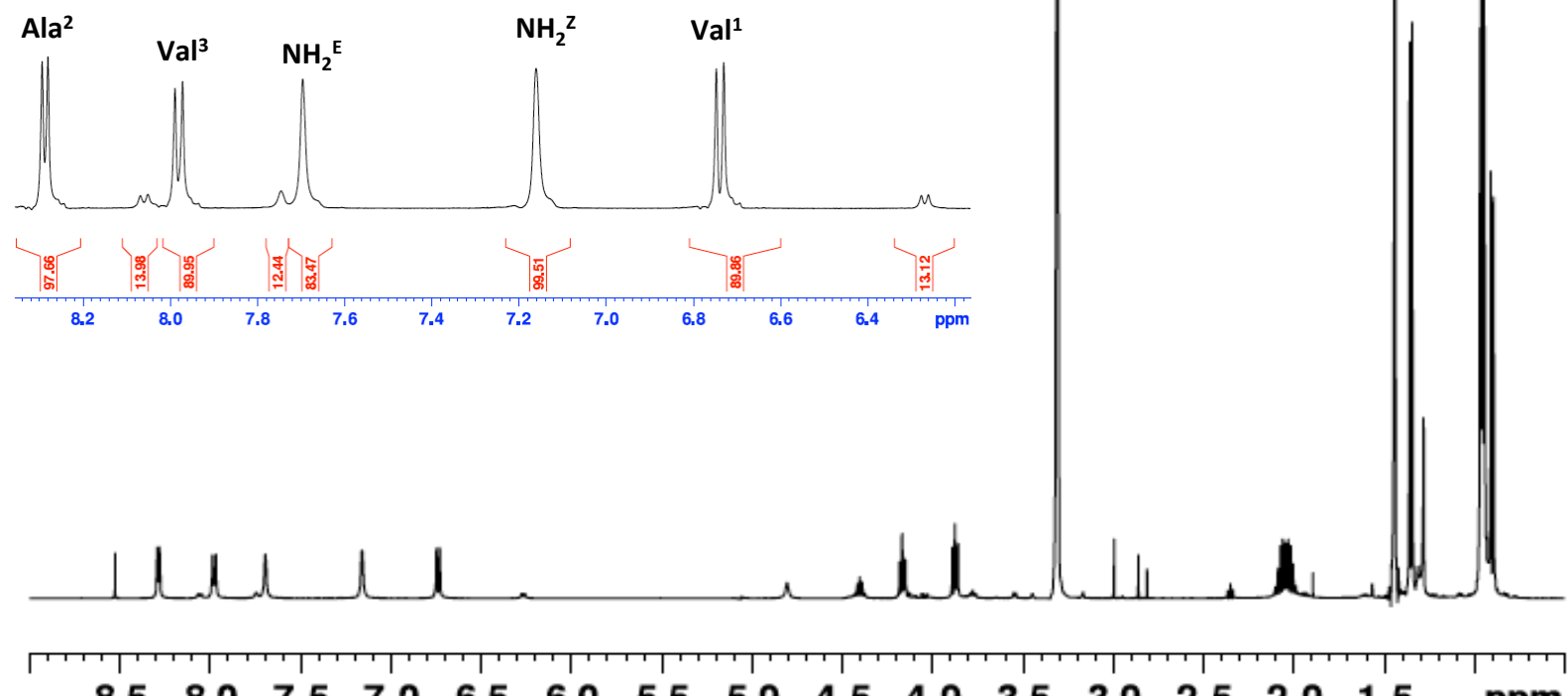

$\begin{array}{lllllllllllllllll}8.5 & 8.0 & 7.5 & 7.0 & 6.5 & 6.0 & 5.5 & 5.0 & 4.5 & 4.0 & 3.5 & 3.0 & 2.5 & 2.0 & 1.5 & \mathrm{ppm}\end{array}$

Figure S1: $1 \mathrm{D}^{1} \mathrm{H}$ NMR spectrum of peptide $1(4 \mathrm{mM})$ in methanol at $278.4 \mathrm{~K}(500.3 \mathrm{MHz})$ 물

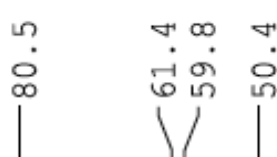

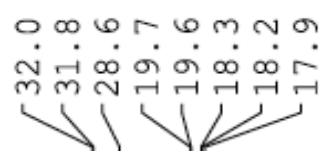<smiles>CC(C)[C@H](NC(=O)[C@H](C)NC(=O)[C@H](NC(=O)OC(C)(C)C)C(C)C)C(N)=O</smiles>
Boc V1 $\quad$ A2 $\quad$ V3

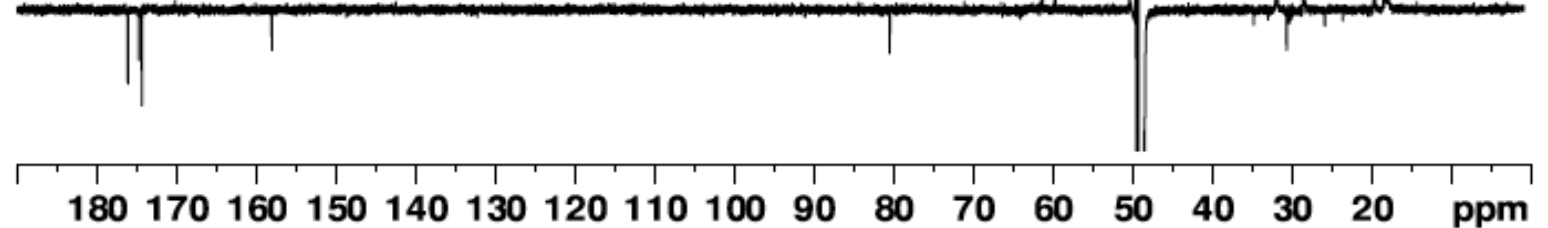

Figure S2: $1 \mathrm{D}{ }^{13} \mathrm{C}$ DEPTQ NMR spectrum of peptide $1(4 \mathrm{mM})$ in methanol at $278.4 \mathrm{~K}(125.8 \mathrm{MHz})$ 

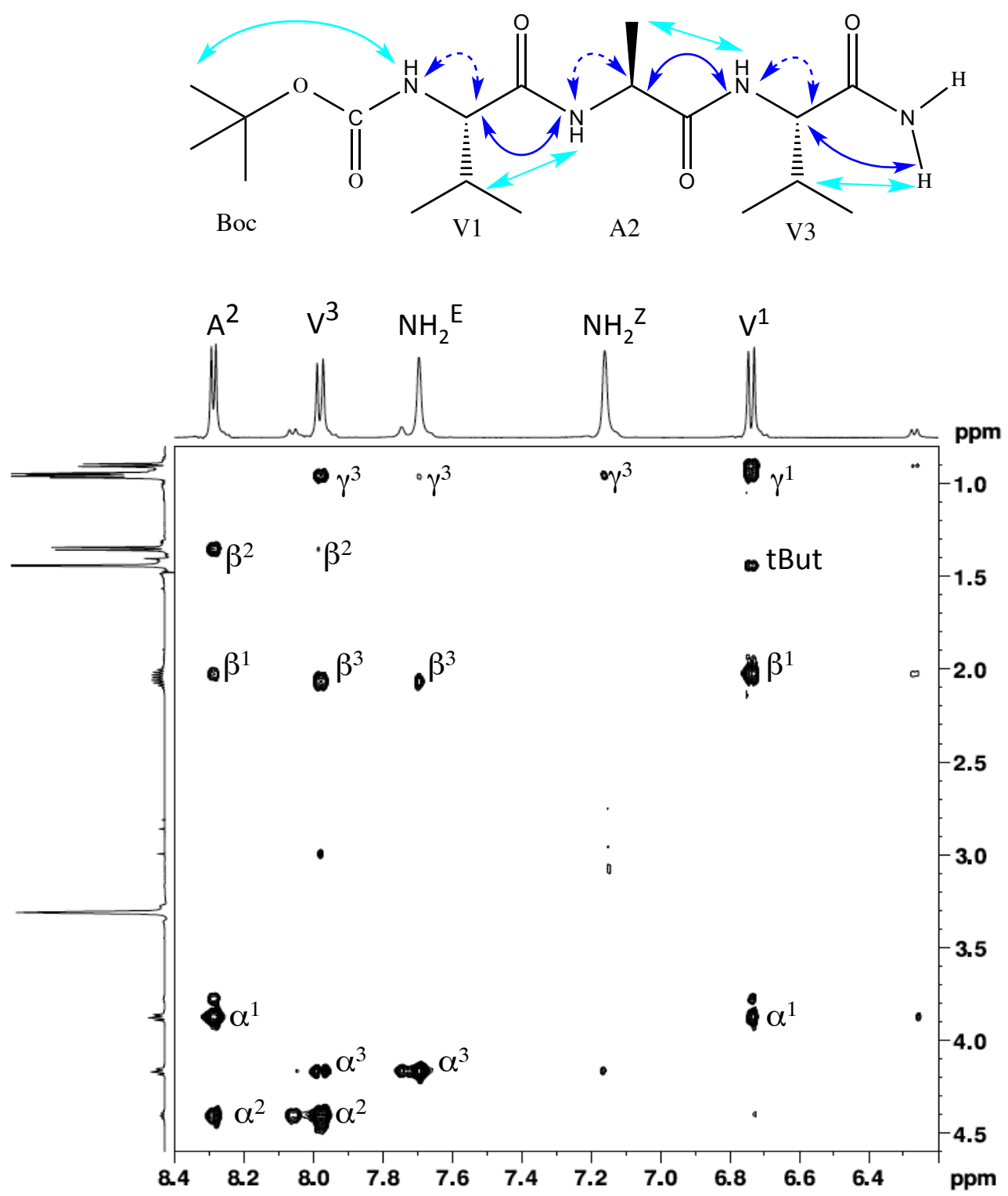

Figure S3: Expansion of the 2D ${ }^{1} \mathrm{H}-{ }^{1} \mathrm{H}$ ROESY spectrum of pseudopeptide $1(4 \mathrm{mM})$ in methanol at $278.4 \mathrm{~K}$ (500.3 MHz, ROE mixing time of $800 \mathrm{~ms}$ ), showing the sequential and intra-residue correlation peaks (plain and dash blue arrows correspond to strong/medium intensity respectively, cyan arrows correspond to weaker intensity). 
NMR data for compound 2 (Boc-aVal-aAla-Val- $\mathrm{NH}_{2}$ )

Table S4. ${ }^{1} \mathrm{H}$ NMR chemical shifts for the two conformers (A, B) of pseudotripeptide $2(28.6 \mathrm{mM})$ in $\mathrm{CD}_{3} \mathrm{OH}$ at $273 \mathrm{~K}$

\begin{tabular}{|c|c|c|c|c|}
\hline Residue & $\delta \mathrm{HN}(\mathrm{ppm}),{ }^{3} \mathrm{~J}(\mathrm{~Hz})$ & $\delta H^{\alpha}(p p m)$ & $\delta H^{\beta}(p p m)$ & $\delta$ Other protons (ppm) \\
\hline \multirow[t]{2}{*}{ Boc } & & & & $\mathrm{CH}_{3} 1.52(\mathrm{~A})$ \\
\hline & & & & $\mathrm{CH}_{3} 1.51$ (B) \\
\hline \multirow[t]{2}{*}{$\mathrm{aVal}^{1}$} & $9.26(A)$ & & $4.55(\mathrm{~A})$ & $\gamma \mathrm{CH}_{3} 1.12, \gamma^{\prime} \mathrm{CH}_{3} 1.08(\mathrm{~A})$ \\
\hline & 9.16 (B) & & 4.54 (B) & $\gamma \mathrm{CH}_{3} 1.11, \gamma^{\prime} \mathrm{CH}_{3} 1.04$ (B) \\
\hline \multirow[t]{2}{*}{$\mathrm{aAla}^{2}$} & $9.65(\mathrm{~A})$ & & $3.07(\mathrm{~A})$ & \\
\hline & 9.35 (B) & & $3.08(\mathrm{~B})$ & \\
\hline \multirow[t]{2}{*}{$\mathrm{Val}^{3}$} & $6.55(A)\left(d,{ }^{3} J=9.0\right)$ & $4.08(\mathrm{~A})$ & $2.33(\mathrm{~A})$ & $\gamma \mathrm{CH}_{3} 0.987, \gamma^{\prime} \mathrm{CH}_{3} 0.98$ (A) \\
\hline & $6.52(B)\left(d,{ }^{3} J=8.4\right)$ & 3.98 (B) & 2.17 (B) & $\gamma \mathrm{CH}_{3} 0.990, \gamma^{\prime} \mathrm{CH}_{3} 0.98$ (B) \\
\hline \multirow[t]{2}{*}{$\mathrm{NH}_{2} \mathrm{Z,E}$} & $7.35,7.39(\mathrm{~A})$ & & & \\
\hline & $7.19,7.54$ (B) & & & \\
\hline
\end{tabular}

Table S5. ${ }^{13} \mathrm{C}$ NMR chemical shifts for the two conformers (A, B) of pseudotripeptide 2 (28.6 mM) in $\mathrm{CD}_{3} \mathrm{OH}$ at $273 \mathrm{~K}$

\begin{tabular}{|c|c|c|c|c|}
\hline Residue & $\delta \mathrm{CO}(\mathrm{ppm})$ & $\delta C^{\alpha}(p p m)$ & $\delta C^{\beta}(p p m)$ & $\delta$ Other carbons (ppm) \\
\hline \multirow[t]{2}{*}{ Boc } & $157.7(A)$ & & & $\mathrm{Cq} 82.4, \mathrm{CH}_{3} 28.4(\mathrm{~A})$ \\
\hline & 157.8 (B) & & & $\mathrm{Cq} 82.3, \mathrm{CH}_{3} 28.5$ (B) \\
\hline \multirow[t]{2}{*}{$\mathrm{aVal}^{1}$} & $158.6(A)$ & & $50.3(\mathrm{~A})$ & $\gamma \mathrm{CH}_{3} 19.8, \gamma^{\prime} \mathrm{CH}_{3} 19.1$ (A) \\
\hline & $158.0(\mathrm{~B})$ & & $50.0(\mathrm{~B})$ & $\gamma \mathrm{CH}_{3} 20.1, \gamma^{\prime} \mathrm{CH}_{3} 19.1$ (B) \\
\hline \multirow[t]{2}{*}{$\mathrm{aAla}^{2}$} & $161.0(\mathrm{~A})$ & & $36.8(\mathrm{~A})$ & \\
\hline & 160.6 (B) & & 36.5 (B) & \\
\hline \multirow[t]{2}{*}{$\mathrm{Val}^{3}$} & $178.2(\mathrm{~A})$ & $61.3(\mathrm{~A})$ & $30.5(\mathrm{~A})$ & $\gamma \mathrm{CH}_{3} 17.3, \gamma^{\prime} \mathrm{CH}_{3} 19.8(\mathrm{~A})$ \\
\hline & $177.8(B)$ & $61.5(\mathrm{~B})$ & $31.1(\mathrm{~B})$ & $\gamma \mathrm{CH}_{3} 18.3, \gamma^{\prime} \mathrm{CH}_{3} 19.9$ (B) \\
\hline
\end{tabular}

Table S6. NMR conformational parameters for 2 in $\mathrm{CD}_{3} \mathrm{OH}$

\begin{tabular}{ccc}
\hline Residue & $\Delta \delta \mathrm{HN} / \Delta \mathrm{T}(\mathrm{ppb} / \mathrm{K})$ & ${ }^{3} \mathrm{JHN}-\mathrm{H \alpha}(\mathrm{Hz})^{*}$ \\
\hline $\mathrm{aVal}^{1}$ & $-7.3(\mathrm{~A})$ & \\
& $-7.1(\mathrm{~B})$ & \\
$\mathrm{aAla}^{2}$ & $-6.55(\mathrm{~A})$ & \\
& $-6.8(\mathrm{~B})$ & $9.0(\mathrm{~A})$ \\
$\mathrm{Val}^{3}$ & $-2.6(\mathrm{~A})$ & $8.4(\mathrm{~B})$ \\
& $-2.3(\mathrm{~B})$ & \\
$\mathrm{NH}_{2, \mathrm{E}}$ & $-8.4,-3.3(\mathrm{~A})$ & \\
& $-7.3,-6.2(\mathrm{~B})$ & \\
\hline
\end{tabular}

$*$ measured at $247.5 \mathrm{~K}$ 


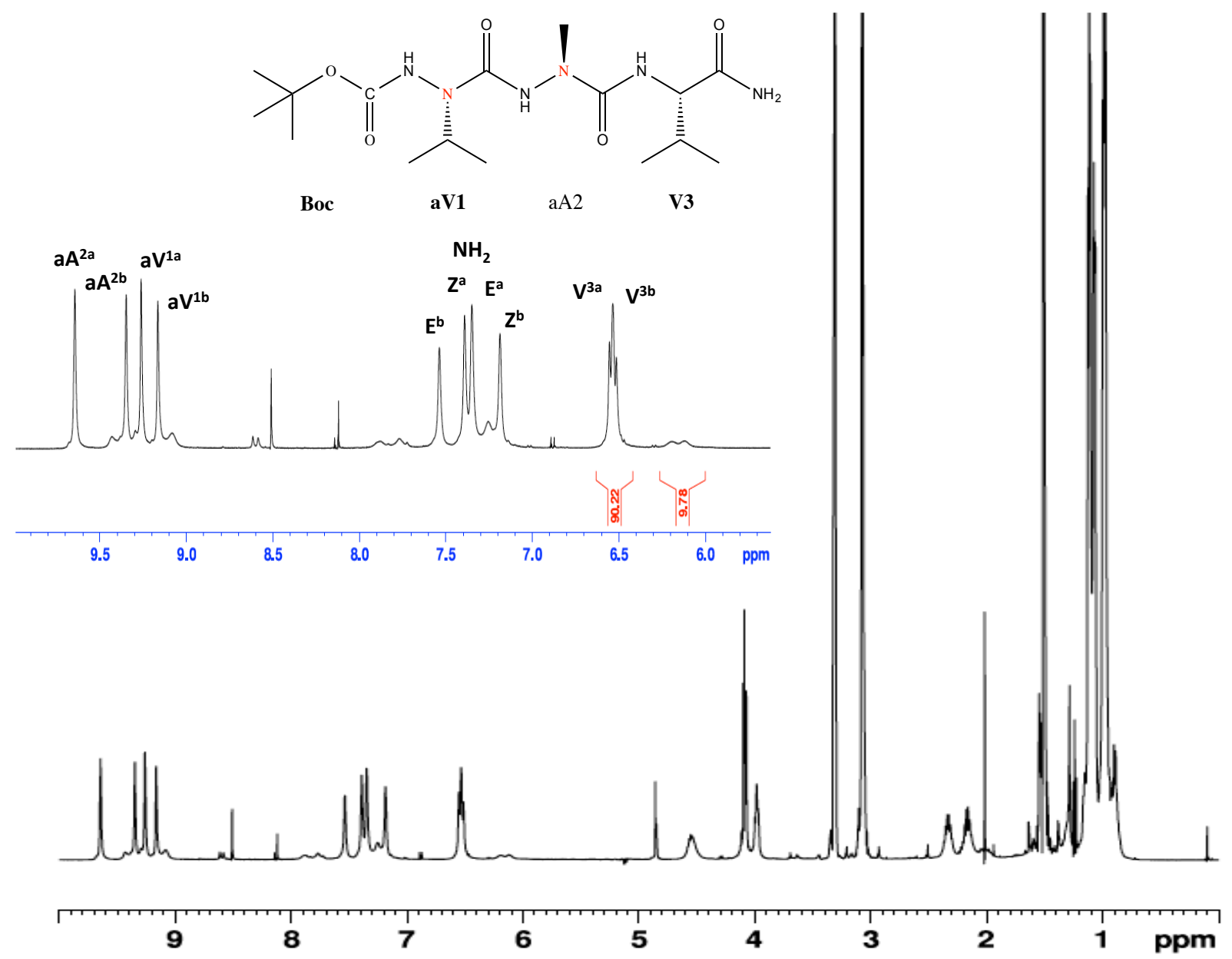

Figure S4: $1 \mathrm{D}{ }^{1} \mathrm{H}$ NMR spectrum of pseudopeptide $2(28.6 \mathrm{mM})$ in methanol at $273 \mathrm{~K}(500.3 \mathrm{MHz})$

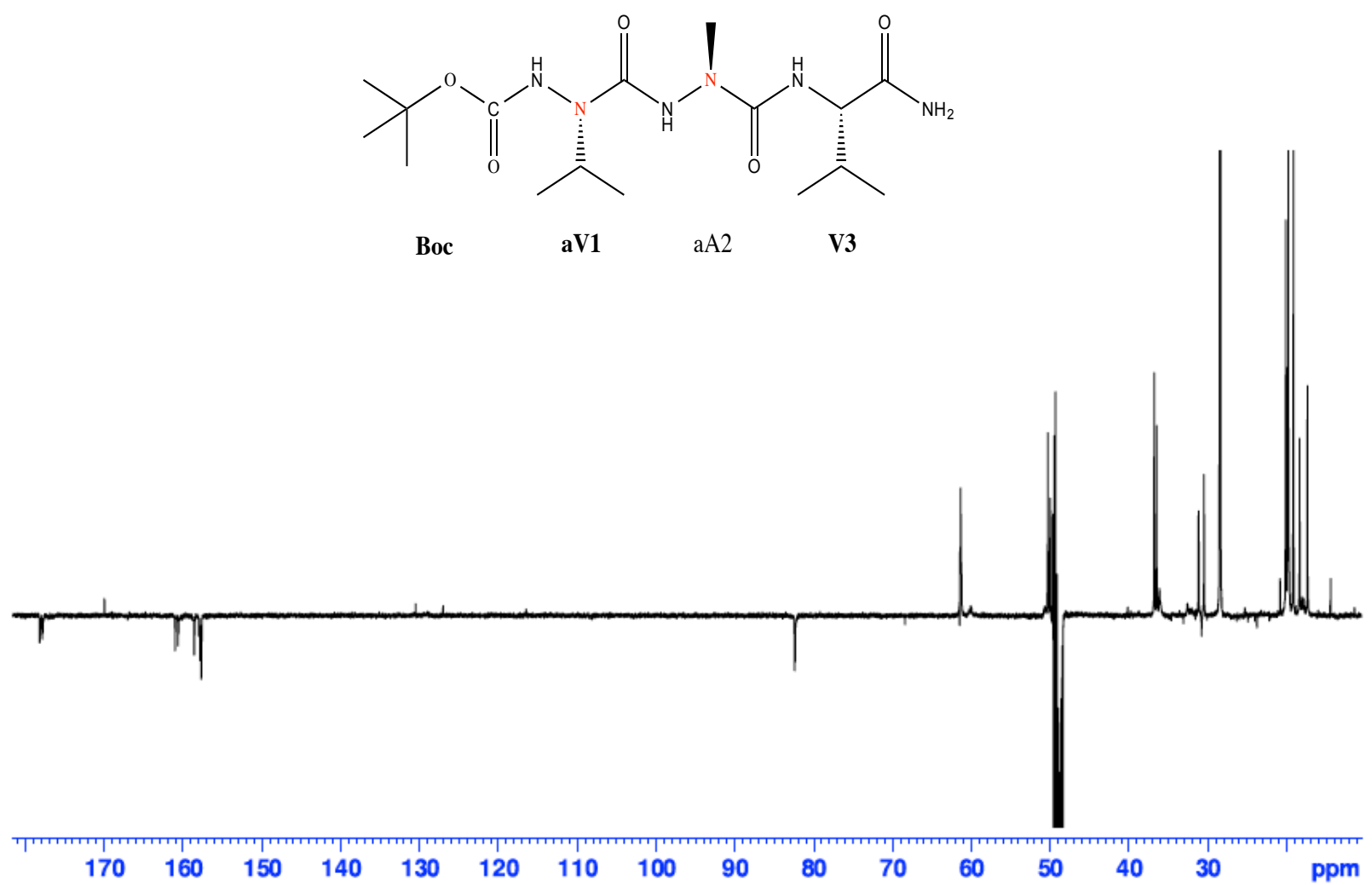

Figure S5: $1 \mathrm{D}{ }^{13} \mathrm{C}$ DEPTQ NMR spectrum of pseudopeptide $2(28.6 \mathrm{mM})$ in methanol at $273 \mathrm{~K}(125.8 \mathrm{MHz})$ 


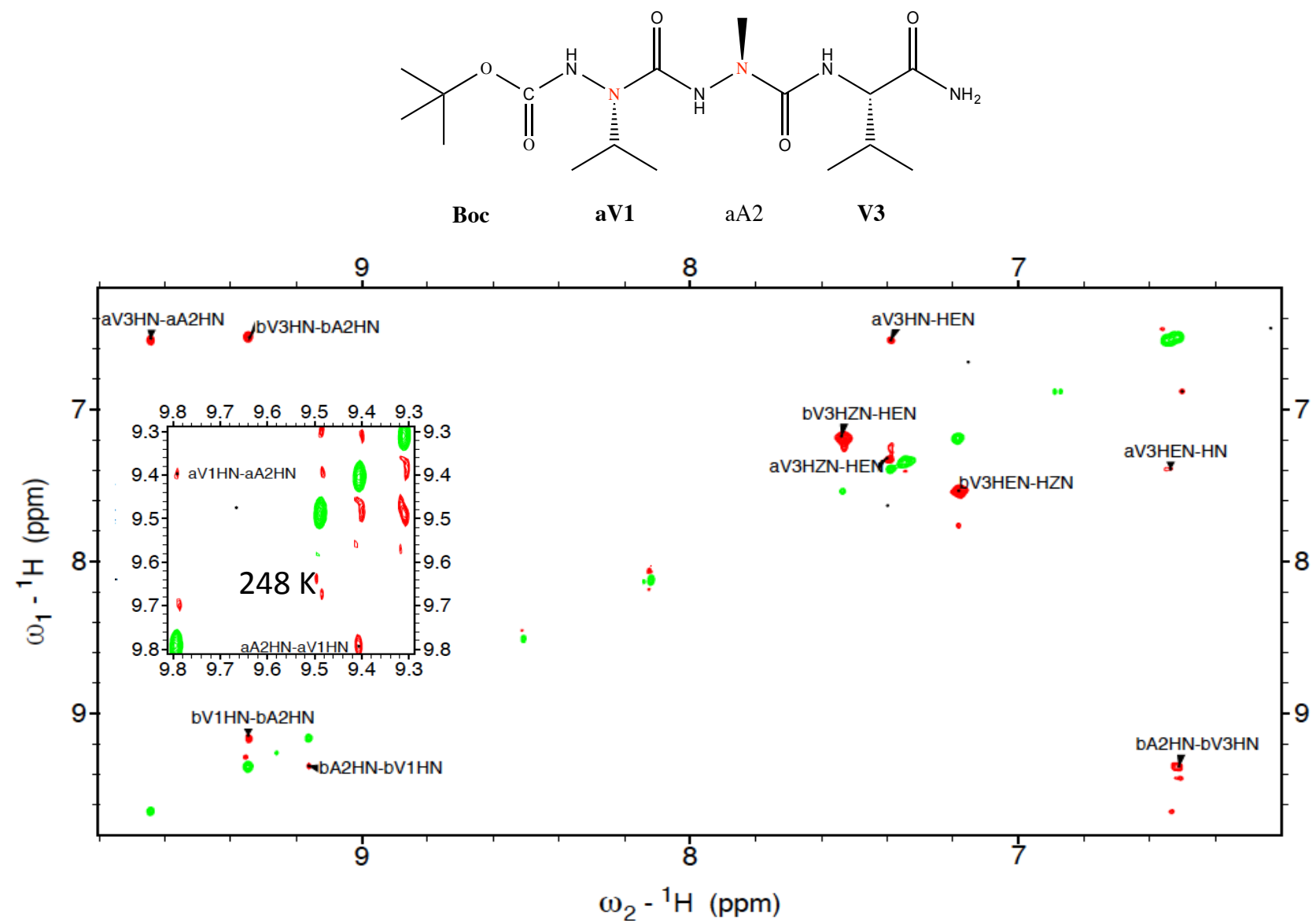

Figure S6: Expansion of the 2D ${ }^{1} \mathrm{H}-{ }^{1} \mathrm{H}$ ROESY spectrum of pseudopeptide $2(28.6 \mathrm{mM})$ in methanol at $273 \mathrm{~K}(500.3 \mathrm{MHz}$, mixing time of $800 \mathrm{~ms}$ ), showing the sequential correlation peaks $\mathrm{NHi}-\mathrm{NHi}+1$ (in red) for conformers $\mathrm{A}$ and $\mathrm{B}$. The insert shows the sequential ROE observed for $\mathrm{aV} 1$ and aV2 residues of conformer A at $248 \mathrm{~K}$ (which is not observed at $273 \mathrm{~K}$ ).

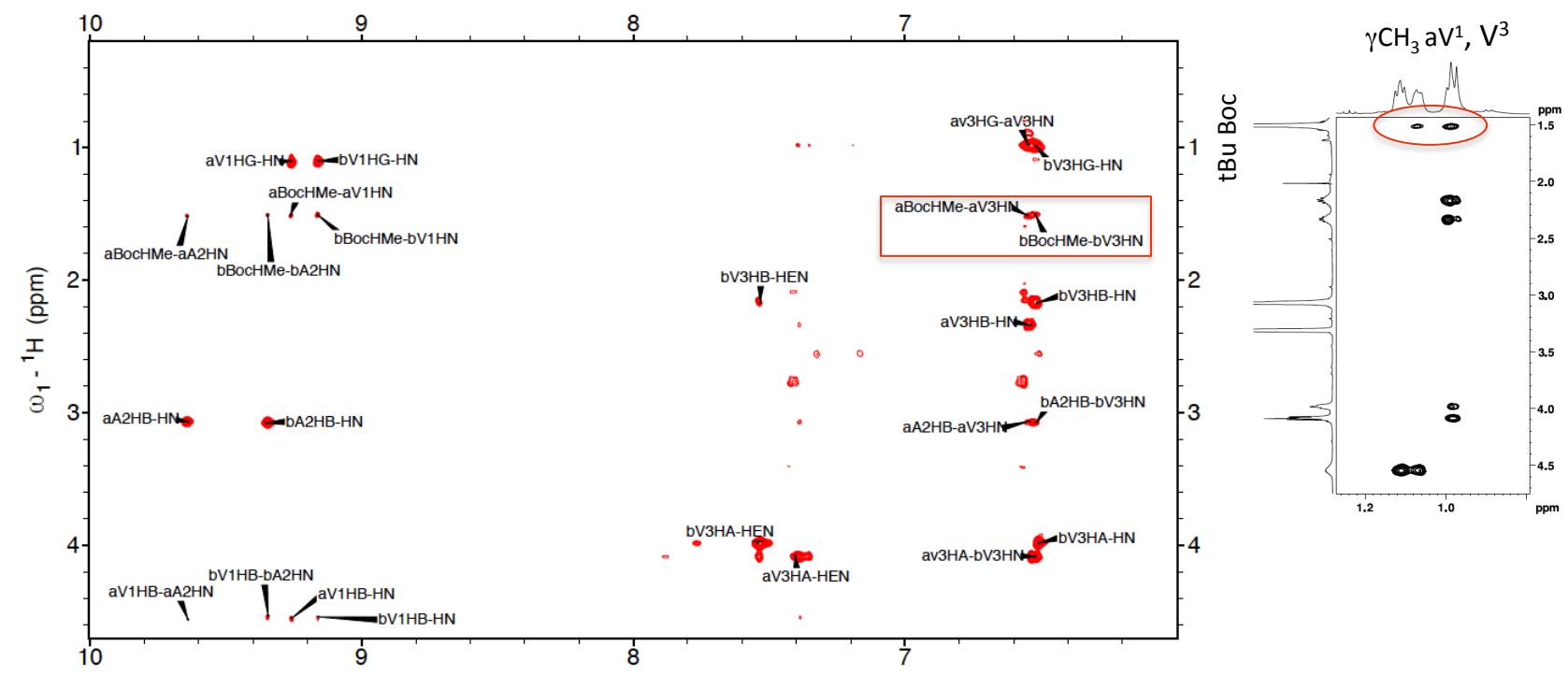

$\omega_{2}-{ }^{1} \mathrm{H}(\mathrm{ppm})$

Figure S7: Expansion of the 2D ${ }^{1} \mathrm{H}-{ }^{1} \mathrm{H}$ ROESY spectrum of pseudopeptide $2(28.6 \mathrm{mM})$ in methanol at $273 \mathrm{~K}$ (500.3 MHz, mixing time of $800 \mathrm{~ms}$ ), showing the correlation peaks between $\mathrm{NH}$ and side chain protons for conformers $\mathrm{A}$ and $\mathrm{B}$. The insert shows additional long-distance ROEs indicating the folding of conformers $\mathrm{A}$ and $\mathrm{B}$. 
NMR data for compound 3 (Boc-aVal-aAla-Val-OBn)

Table S7. ${ }^{1} \mathrm{H}$ NMR chemical shifts for the two conformers of pseudotripeptide 3 ( $28.6 \mathrm{mM}$ ) in $\mathrm{CD}_{3} \mathrm{OH}$ at $273 \mathrm{~K}$

\begin{tabular}{|c|c|c|c|c|}
\hline Residue & $\delta \mathrm{HN}(\mathrm{ppm}),{ }^{3} \mathrm{~J}(\mathrm{~Hz})$ & $\delta H^{a}(p p m)$ & $\delta \mathrm{H}^{\beta}(\mathrm{ppm})$ & $\delta$ Other protons (ppm) \\
\hline \multirow[t]{2}{*}{ Boc } & & & & $\mathrm{CH}_{3} 1.49(\mathrm{~A})$ \\
\hline & & & & $\mathrm{CH}_{3} 1.50$ (B) \\
\hline \multirow[t]{2}{*}{$\mathrm{aVal}^{1}$} & $9.13(\mathrm{~A})$ & & $4.53(A, B)$ & $\gamma \mathrm{CH}_{3} 1.11, \gamma^{\prime} \mathrm{CH}_{3} 1.06(\mathrm{~A}, \mathrm{~B})$ \\
\hline & 9.08 (B) & & & \\
\hline \multirow[t]{2}{*}{$\mathrm{aAla}^{2}$} & $9.43(\mathrm{~A})$ & & $3.08(\mathrm{~A})$ & \\
\hline & 9.38 (B) & & 3.07 (B) & \\
\hline \multirow[t]{2}{*}{$\mathrm{Val}^{3}$} & $6.71(\mathrm{~A}, \mathrm{~B})(\mathrm{d}, J=8.8)$ & $4.12(A, B)$ & $2.18(\mathrm{~A})$ & $\gamma \mathrm{CH}_{3} 0.96, \gamma^{\prime} \mathrm{CH}_{3} 0.93(\mathrm{~A})$ \\
\hline & & & $2.11(B)$ & $\gamma \mathrm{CH}_{3} 0.93, \gamma^{\prime} \mathrm{CH}_{3} 0.89$ (B) \\
\hline $\mathrm{OBz}$ & & & & $\mathrm{CH}_{2}(5.17,5.10), \mathrm{Ph} 7.4(\mathrm{~A}, \mathrm{~B})$ \\
\hline
\end{tabular}

Table S8. ${ }^{13} \mathrm{C}$ NMR chemical shifts for the two conformers of pseudotripeptide $3(28.6 \mathrm{mM})$ in $\mathrm{CD}_{3} \mathrm{OH}$ at $273 \mathrm{~K}$

\begin{tabular}{|c|c|c|c|c|}
\hline Residue & $\delta \mathrm{CO}(\mathrm{ppm})$ & $\delta C^{\alpha}(p p m)$ & $\delta C^{\beta}(p p m)$ & $\delta$ Other carbons (ppm) \\
\hline \multirow[t]{2}{*}{ Boc } & $157.6(\mathrm{~A})$ & & & $\mathrm{Cq} 82.2, \mathrm{CH}_{3} 28.5(\mathrm{~A})$ \\
\hline & $157.7(B)$ & & & $\mathrm{Cq} 82.0, \mathrm{CH}_{3} 28.4$ (B) \\
\hline \multirow[t]{2}{*}{$\mathrm{aVal}^{1}$} & $158.02(\mathrm{~A})$ & & $49.9(A, B)$ & $\gamma \mathrm{CH}_{3} 20.1, \gamma^{\prime} \mathrm{CH}_{3} 19.06$ (A, B) \\
\hline & $157.97(B)$ & & & \\
\hline \multirow[t]{2}{*}{$\mathrm{aAla}^{2}$} & $160.7(A)$ & & $36.3(A, B)$ & \\
\hline & 160.6 (B) & & & \\
\hline \multirow[t]{2}{*}{$\mathrm{Val}^{3}$} & $174.0(A, B)$ & $60.9(A)$ & $31.4(\mathrm{~A})$ & $\gamma \mathrm{CH}_{3} 18.6, \gamma^{\prime} \mathrm{CH}_{3} 19.6$ (A) \\
\hline & & $60.8(B)$ & 31.9 (B) & $\gamma \mathrm{CH}_{3} 19.13, \gamma^{\prime} \mathrm{CH}_{3} 19.4$ (B) \\
\hline $\mathrm{OBz}$ & & & & $\mathrm{CH}_{2}$ 67.4, $\mathrm{Ph}(137.2,129.36,129.40,129.2)$ \\
\hline
\end{tabular}

Table S9. NMR conformational parameters for 3 in $\mathrm{CD}_{3} \mathrm{OH}$

\begin{tabular}{ccc}
\hline Residue & $\Delta \delta \mathrm{HN} / \Delta \mathrm{T}(\mathrm{ppb} / \mathrm{K})$ & ${ }^{3} \mathrm{JHN}-\mathrm{H \alpha}(\mathrm{Hz}){ }^{*}$ \\
\hline $\mathrm{aVal}^{1}$ & $-7.0(\mathrm{~A})-6.92(\mathrm{~B})$ & \\
$\mathrm{aAla}^{2}$ & $-7.75(\mathrm{~A})-7.69(\mathrm{~B})$ & \\
$\mathrm{Val}^{3}$ & $-3.9(\mathrm{~A}, \mathrm{~B})$ & $8.8(\mathrm{~A}, \mathrm{~B})$ \\
\hline
\end{tabular}

$*$ measured at $251 \mathrm{~K}$

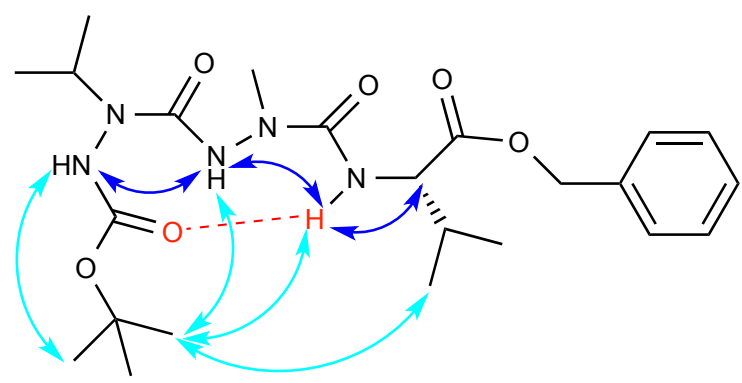

Figure S8. Schematic representation of NMR parameters obtained for Boc-aVal-aAla-Val-OBn 3. ROEs are indicated by blue arrows and hydrogen bond by red line. Similar ROEs are observed for the two major conformers. 
NMR data for compound 4 (Boc-aGly-aGly-Val--NH ${ }_{2}$ )

Table S10. ${ }^{1} \mathrm{H}$ NMR chemical shifts of pseudotripeptide $4(15 \mathrm{mM})$ in $\mathrm{CD}_{3} \mathrm{OH}$ at $293 \mathrm{~K}$

\begin{tabular}{ccccc}
\hline Residue & $\delta \mathrm{HN}(\mathrm{ppm})$ & $\delta \mathrm{H}^{\alpha}(\mathrm{ppm})$ & $\delta \mathrm{H}^{\beta}(\mathrm{ppm})$ & $\delta$ Other protons $(\mathrm{ppm})$ \\
\hline $\mathrm{Boc}$ & & & & \\
$\mathrm{aGly}^{1}$ & $8.56(\mathrm{br})$ & & \\
$\mathrm{aGly}^{2}$ & $7.91(\mathrm{br})$ & & \\
$\mathrm{Val}^{3}$ & $6.47(\mathrm{br})$ & $4.11\left(\mathrm{dd},{ }^{3} J=8.7,6.1 \mathrm{~Hz}\right)$ & $2.16(\mathrm{~m})$ & $\gamma \mathrm{CH}_{3} 0.99\left(\mathrm{~d},{ }^{3} J=7.0 \mathrm{~Hz}\right)$ \\
& & & $\gamma^{\prime} \mathrm{CH}_{3} 0.95\left(\mathrm{~d},{ }^{3} J=7.2 \mathrm{~Hz}\right)$ \\
$\mathrm{NH}_{2 \mathrm{z}, \mathrm{E}}$ & $7.09(\mathrm{~s}), 7.62(\mathrm{br})$ & & \\
\hline br: broad & &
\end{tabular}

br: broad

Table S11. ${ }^{13} \mathrm{C}$ NMR chemical shifts of pseudotripeptide $4(15 \mathrm{mM})$ in $\mathrm{CD}_{3} \mathrm{OH}$ at $293 \mathrm{~K}$

\begin{tabular}{ccccc}
\hline Residue & $\delta \mathrm{CO}(\mathrm{ppm})$ & $\delta \mathrm{C}^{\alpha}(\mathrm{ppm})$ & $\delta \mathrm{C}^{\beta}(\mathrm{ppm})$ & $\delta$ Other carbons $(\mathrm{ppm})$ \\
\hline Boc & 158.3 & & & $\mathrm{Cq} 81.9, \mathrm{CH}_{3} 28.4$ \\
$\mathrm{aGly}^{1}$ & 161.3 & & & \\
$\mathrm{aGly}^{2}$ & 160.9 & & 31.6 & $\gamma \mathrm{CH}_{3} 19.7, \gamma^{\prime} \mathrm{CH}_{3} 17.9$ \\
Val $^{3}$ & 177.3 & 60.3 & 31.6 \\
\hline
\end{tabular}

Table S12. Temperature coefficients $(\Delta \delta / \Delta T)$ of the amide protons chemical shifts of pseudotripeptide 4 in methanol solution.

\begin{tabular}{cc}
\hline Residue & $\Delta \delta H N / \Delta T(p p b / K)$ \\
\hline aGly $^{1}$ & -9.4 \\
aGly $^{2}$ & -6.3 \\
Val $^{3}$ & -4.2 \\
$\mathrm{NH}_{2 \text { Z, E }}$ & $-6.1,-8.6$ \\
\hline
\end{tabular}




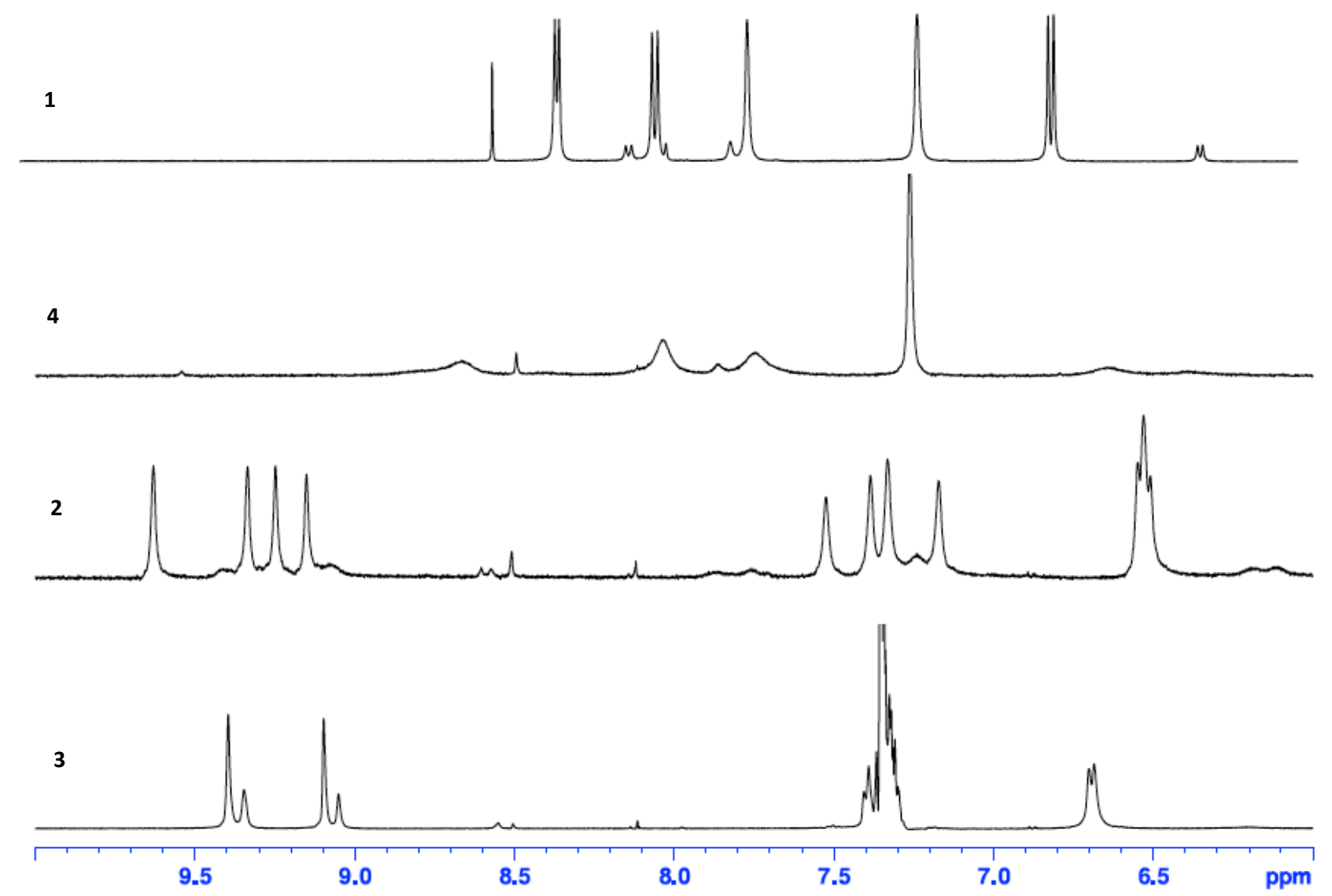

Figure S9: Comparison of peptide 1 amide region with that of pseudopeptides 2, 3 and 4, in methanol at $273 \mathrm{~K}(500.3 \mathrm{MHz})$ 
NMR data for compound 5 (Boc-aVal-aAla-Val-aVal-aAla-Val-NH ${ }_{2}$ )

Table S13. ${ }^{1} \mathrm{H}$ NMR chemical shifts for the two major conformers of pseudopeptide $5(15 \mathrm{mM})$ in $\mathrm{CD}_{3} \mathrm{OH}$ at $273 \mathrm{~K}$

\begin{tabular}{|c|c|c|c|c|}
\hline Residue & $\delta \mathrm{HN}(\mathrm{ppm}){ }^{3} \mathrm{~J}(\mathrm{~Hz})$ & $\delta \mathrm{H}^{\alpha}(\mathrm{ppm})$ & $\delta H^{\beta}(p p m)$ & $\delta$ Other protons (ppm) \\
\hline \multirow[t]{2}{*}{ Boc } & & & & $\mathrm{CH}_{3} 1.50(\mathrm{~A})$ \\
\hline & & & & $\mathrm{CH}_{3} 1.53(\mathrm{~B})$ \\
\hline \multirow[t]{2}{*}{$\mathrm{aVal}^{1}$} & $9.10(\mathrm{~A})$ & & $4.56(\mathrm{~A})$ & $\gamma \mathrm{CH}_{3} 1.10, \gamma^{\prime} \mathrm{CH}_{3} 1.08(\mathrm{~A})$ \\
\hline & $9.40(B)$ & & $4.61(B)$ & $\gamma \mathrm{CH}_{3} 1.12, \gamma^{\prime} \mathrm{CH}_{3} 1.12(\mathrm{~B})$ \\
\hline \multirow[t]{2}{*}{$\mathrm{aAla}^{2}$} & $9.62(\mathrm{~A})$ & & $2.88(\mathrm{~A})$ & \\
\hline & $9.87(\mathrm{~B})$ & & $3.09(\mathrm{~B})$ & \\
\hline \multirow[t]{2}{*}{$\mathrm{Val}^{3}$} & $6.78(\mathrm{~A})\left(\mathrm{d},{ }^{3} \mathrm{~J}=6.5\right)$ & $3.62(A)$ & $2.10(A)$ & $\gamma \mathrm{CH}_{3} 1.12, \gamma^{\prime} \mathrm{CH}_{3} 1.05(\mathrm{~A})$ \\
\hline & $6.86(B)\left(d,{ }^{3} J=5.2\right)$ & $3.96(B)$ & $2.27(\mathrm{~B})$ & $\gamma \mathrm{CH}_{3} 1.116, \gamma^{\prime} \mathrm{CH}_{3} 1.116(\mathrm{~B})$ \\
\hline \multirow[t]{2}{*}{$\mathrm{aVal}^{4}$} & $10.54(\mathrm{~A})$ & & $4.52(\mathrm{~A})$ & $\gamma \mathrm{CH}_{3} 1.14, \gamma^{\prime} \mathrm{CH}_{3} 1.06(\mathrm{~A})$ \\
\hline & $9.71(\mathrm{~B})$ & & $4.60(B)$ & $\gamma \mathrm{CH}_{3} 1.17, \gamma^{\prime} \mathrm{CH}_{3} 1.11$ (B) \\
\hline \multirow[t]{2}{*}{$\mathrm{aAla}^{5}$} & $9.18(\mathrm{~A})$ & & $3.10(A)$ & \\
\hline & $9.05(B)$ & & 3.09 (B) & \\
\hline \multirow[t]{2}{*}{$\mathrm{Val}^{6}$} & $6.39(A)\left(d,{ }^{3} J=9.3\right)$ & $3.85(A)$ & $2.30(A)$ & $\gamma \mathrm{CH}_{3} 0.95, \gamma^{\prime} \mathrm{CH}_{3} 0.94(\mathrm{~A})$ \\
\hline & $6.43(B)\left(d,{ }^{3} J=8.2\right)$ & $4.07(B)$ & $2.33(B)$ & $\gamma \mathrm{CH}_{3} 1.00, \gamma^{\prime} \mathrm{CH}_{3} 1.00$ (B) \\
\hline \multirow[t]{2}{*}{$\mathrm{NH}_{2 \mathrm{Z}, \mathrm{E}}$} & $6.83,7.60(\mathrm{~A})$ & & & \\
\hline & $7.35,7.43(B)$ & & & \\
\hline
\end{tabular}

Table S14. ${ }^{13} \mathrm{C}$ NMR chemical shifts for the two major conformers of pseudopeptide 5 (15 mM) in $\mathrm{CD}_{3} \mathrm{OH}$ at $273 \mathrm{~K}$

\begin{tabular}{|c|c|c|c|c|}
\hline Residue & $\delta \mathrm{CO}(\mathrm{ppm})$ & $\delta C^{\alpha}(p p m)$ & $\delta C^{\beta}(p p m)$ & $\delta$ Other carbons (ppm) \\
\hline \multirow[t]{2}{*}{ Boc } & $157.9(\mathrm{~A})$ & & & $\mathrm{Cq} 82.4, \mathrm{CH}_{3} 28.8(\mathrm{~A})$ \\
\hline & $158.0(B)$ & & & $\mathrm{Cq} 82.2, \mathrm{CH}_{3} 28.5$ (B) \\
\hline \multirow[t]{2}{*}{$\mathrm{aVal}^{1}$} & $158.4(A)$ & & $49.8(\mathrm{~A})$ & $\gamma \mathrm{CH}_{3} 20.1, \gamma^{\prime} \mathrm{CH}_{3} 19.2(\mathrm{~A})$ \\
\hline & $159.1(\mathrm{~B})$ & & $50.1(\mathrm{~B})$ & $\gamma \mathrm{CH}_{3} 20.1, \gamma^{\prime} \mathrm{CH}_{3} 20.1(\mathrm{~B})$ \\
\hline \multirow[t]{2}{*}{$\mathrm{aAla}^{2}$} & $161.9(A)$ & & $36.5(\mathrm{~A})$ & \\
\hline & $162.6(B)$ & & $37.0(\mathrm{~B})$ & \\
\hline \multirow[t]{2}{*}{$\mathrm{Val}^{3}$} & $176.8(\mathrm{~A})$ & $62.6(\mathrm{~A})$ & $30.1(\mathrm{~A})$ & $\gamma \mathrm{CH}_{3} 20.1, \gamma^{\prime} \mathrm{CH}_{3} 20.3(\mathrm{~A})$ \\
\hline & $174.8(B)$ & $63.7(\mathrm{~B})$ & 30.7 (B) & $\gamma \mathrm{CH}_{3} 19.3, \gamma^{\prime} \mathrm{CH}_{3} 19.3$ (B) \\
\hline \multirow[t]{2}{*}{$\mathrm{aVal}^{4}$} & $157.2(A)$ & & $49.7(A)$ & $\gamma \mathrm{CH}_{3} 20.1, \gamma^{\prime} \mathrm{CH}_{3} 20.3(\mathrm{~A})$ \\
\hline & $157.7(B)$ & & $50.2(\mathrm{~B})$ & $\gamma \mathrm{CH}_{3} 20.2, \gamma^{\prime} \mathrm{CH}_{3} 20.2$ (B) \\
\hline \multirow[t]{2}{*}{$\mathrm{aAla}^{5}$} & $160.3(\mathrm{~A})$ & & $37.0(\mathrm{~A})$ & \\
\hline & 160.6 (B) & & 37.0 (B) & \\
\hline \multirow[t]{2}{*}{$\mathrm{Val}^{6}$} & $176.5(A)$ & $61.4(\mathrm{~A})$ & $31.0(\mathrm{~A})$ & $\gamma \mathrm{CH}_{3} 20.14, \gamma^{\prime} \mathrm{CH}_{3} 19.18$ (A) \\
\hline & $178.2(\mathrm{~B})$ & $61.6(B)$ & $30.8(B)$ & $\gamma \mathrm{CH}_{3} 17.6, \gamma^{\prime} \mathrm{CH}_{3} 19.8$ (B) \\
\hline
\end{tabular}




\begin{tabular}{|c|c|c|c|c|}
\hline \multirow[t]{2}{*}{ Distance } & \multicolumn{2}{|c|}{ Conformer 5A } & \multicolumn{2}{|c|}{ Conformer 5B } \\
\hline & $\begin{array}{c}\text { Experimental } \\
\text { (ROE) }\end{array}$ & Calculated & $\begin{array}{c}\text { Experimental } \\
\text { (ROE) }\end{array}$ & Calculated \\
\hline \multicolumn{5}{|l|}{ Intraresidual } \\
\hline aVal1 $H N-H \beta$ & 3.8 & 3.8 & 3.5 & 3.7 \\
\hline aAla2 $\mathrm{HN}-\mathrm{H} \beta$ & 3.5 & 3.3 & 3.6 & 3.3 \\
\hline Val3 HN - H $\alpha$ & 2.7 & 2.9 & 2.7 & 2.9 \\
\hline Val3 HN - H $\beta$ & 2.3 & 2.4 & 2.7 & 2.3 \\
\hline aVal4 $\mathrm{HN}-\mathrm{H} \beta$ & 4.1 & 3.8 & 3.5 & 3.7 \\
\hline aAla5 $\mathrm{HN}-\mathrm{H} \beta$ & 2.9 & 3.3 & 3.3 & 3.3 \\
\hline Val6 $\mathrm{HN}-\mathrm{H} \alpha$ & 2.6 & 2.9 & 3.1 & 3.0 \\
\hline \multicolumn{5}{|l|}{ Sequential } \\
\hline Boc Me - aVal1 HN & 4.3 & 4.5 & 4.0 & 4.9 \\
\hline aVal1 HN - aAla2 HN & 2.8 & 2.8 & 3.3 & 2.9 \\
\hline aVal1 $\mathrm{H} \beta$ - aAla2 HN & 4.0 & 4.4 & 4.9 & 4.4 \\
\hline aAla2 HN - Val3 HN & 2.7 & 2.5 & 3.0 & 2.6 \\
\hline aAla2 H $\beta$ - Val3 HN & 4.5 & 4.5 & 4.4 & 4.5 \\
\hline Val3 HN - aVal4 HN & n.d. & 4.5 & 3.1 & 2.7 \\
\hline Val3 Ho - aVal4 HN & 2.3 & 2.2 & 3.5 & 3.6 \\
\hline Val3 $\mathrm{H} \beta$ - aVal4 HN & n.d. & 4.3 & 4.6 & 3.1 \\
\hline aVal4 HN - aAla5 HN & 2.8 & 2.6 & 2.9 & 2.7 \\
\hline aVal4 $\mathrm{H} \beta$ - aAla5 HN & 4.2 & 4.4 & 4.4 & 4.4 \\
\hline aAla5 HN - Val6 HN & 2.7 & 2.5 & 2.9 & 2.5 \\
\hline aAla5 H $\beta$ - Val6 HN & 4.5 & 4.5 & 4.1 & 4.5 \\
\hline Val6 $\mathrm{HN}-\mathrm{NH}_{2} \mathrm{H}_{\mathrm{E}}$ & 4.1 & 3.8 & 3.3 & 2.1 \\
\hline Val6 $\mathrm{H} \alpha-\mathrm{NH}_{2} \mathrm{H}_{\mathrm{E}}$ & 2.4 & 4.5 & 3.4 & 3.6 \\
\hline \multicolumn{5}{|l|}{ Medium-range } \\
\hline Boc Me - aAla2 HN & 4.7 & 4.2 & 4.9 & 4.0 \\
\hline Boc Me - Val3 HN & 3.8 & 3.6 & 4.1 & 3.8 \\
\hline Boc Me - Val3 H $\beta$ & 2.4 & 2.8 & 4.4 & 4.1 \\
\hline aAla2 $\mathrm{H} \beta$ - aVal4 HN & n.d. & 5.9 & 5.2 & 4.2 \\
\hline aAla2 $\mathrm{H} \beta$ - aAla5HN & 4.0 & 3.7 & ambiguous & 3.5 \\
\hline aAla2 HN - Val6 HN & 4.3 & 4.0 & n.d. & 7.4 \\
\hline aAla2 $\mathrm{H} \beta$ - Val6 HN & 4.4 & 4.5 & n.d. & 5.8 \\
\hline aAla2 $\mathrm{H} \beta$ - Val6 $\mathrm{H} \alpha$ & 4.5 & 5.3 & n.d. & 8.1 \\
\hline Val3 HN - aAla5 HN & 4.3 & 4.2 & n.d. & 4.1 \\
\hline Val3 $\mathrm{H} \alpha-$ aAla5 HN & 3.3 & 3.4 & 3.4 & 3.6 \\
\hline Val3 Ha - Val6 HN & 4.6 & 4.5 & 4.0 & 3.7 \\
\hline \multicolumn{5}{|l|}{ Long-range } \\
\hline Boc $\mathrm{Me}-\mathrm{NH}_{2} \mathrm{H}_{\mathrm{z}}$ & 4.4 & 5.7 & n.d. & 11 \\
\hline aVal1 $\mathrm{HN}-\mathrm{NH}_{2} \mathrm{H}_{\mathrm{Z}}$ & 3.9 & 4.4 & n.d. & 11.6 \\
\hline aAla2 $\mathrm{HN}-\mathrm{NH}_{2} \mathrm{H}_{\mathrm{Z}}$ & 3.2 & 2.8 & n.d. & 10.1 \\
\hline aAla2 $\mathrm{H} \beta-\mathrm{NH}_{2} \mathrm{H}_{\mathrm{Z}}$ & 4.4 & 3.0 & n.d. & 9.3 \\
\hline
\end{tabular}




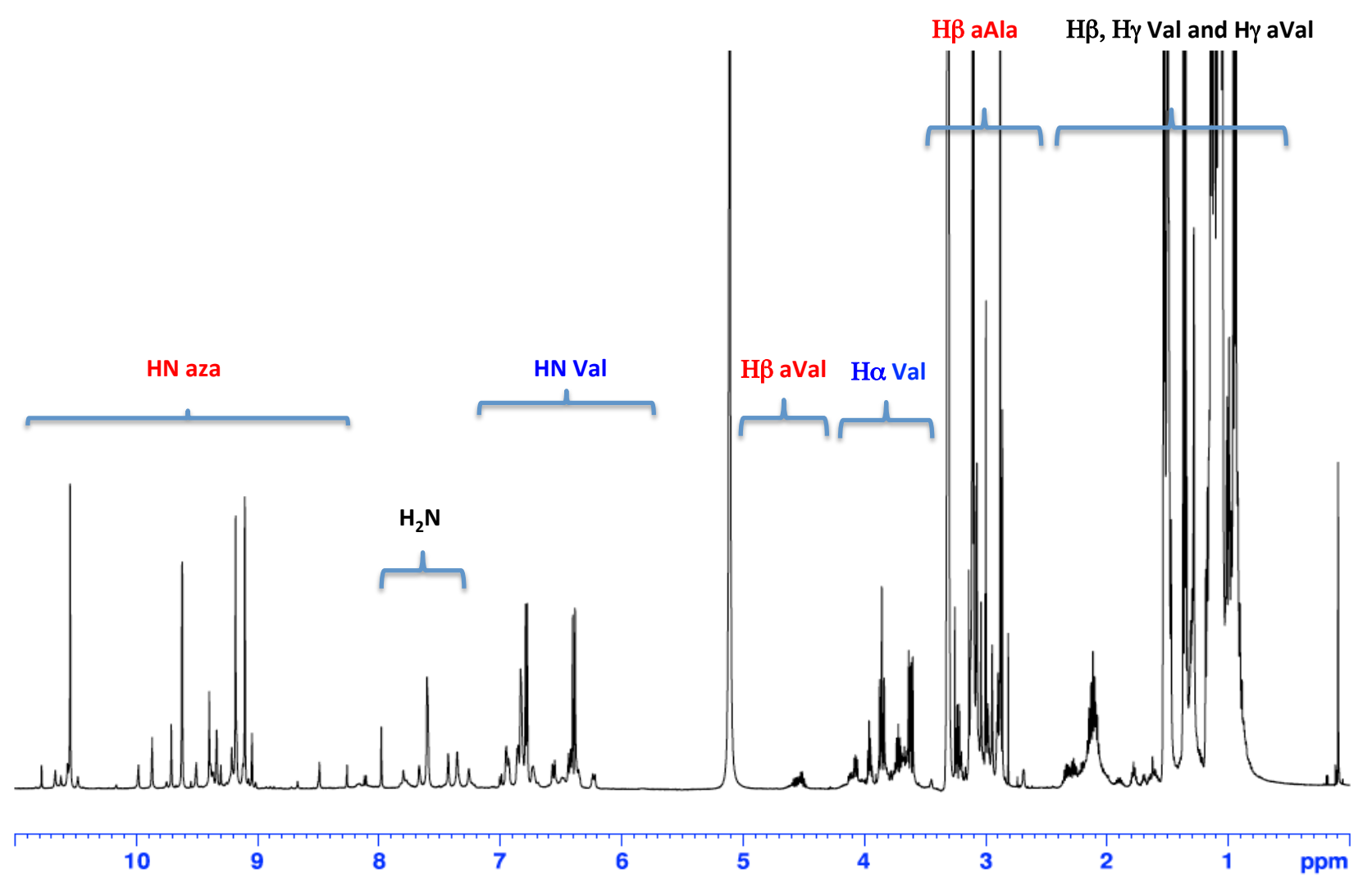

Figure S10: $1 \mathrm{D}{ }^{1} \mathrm{H}$ NMR spectrum of compound $5(15 \mathrm{mM})$ in methanol at $273 \mathrm{~K}(500.3 \mathrm{MHz})$

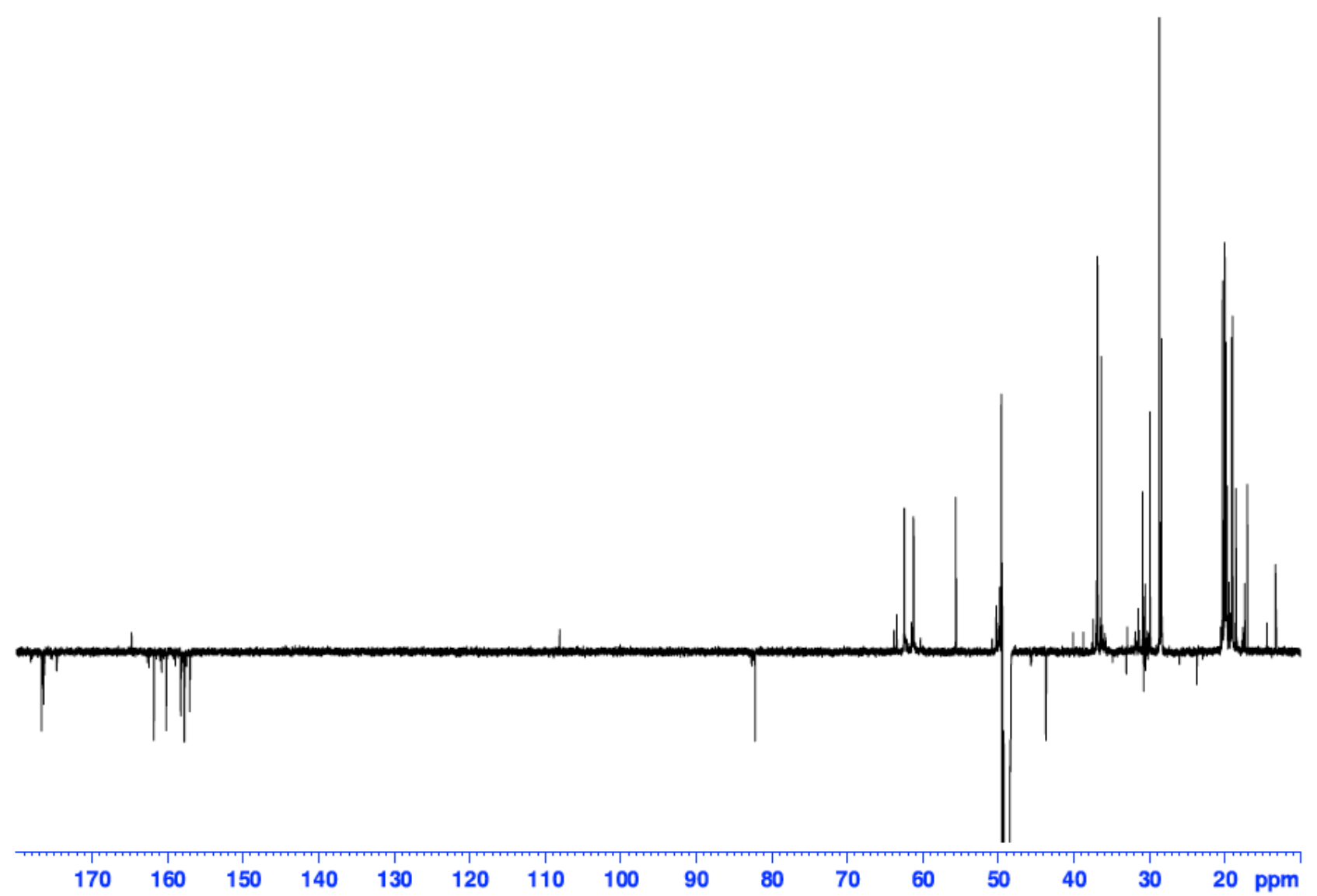

Figure S11: $1 \mathrm{D}{ }^{13} \mathrm{C}$ DEPTQ spectrum of compound $5(15 \mathrm{mM})$ in methanol at $273 \mathrm{~K}(125.8 \mathrm{MHz})$ 


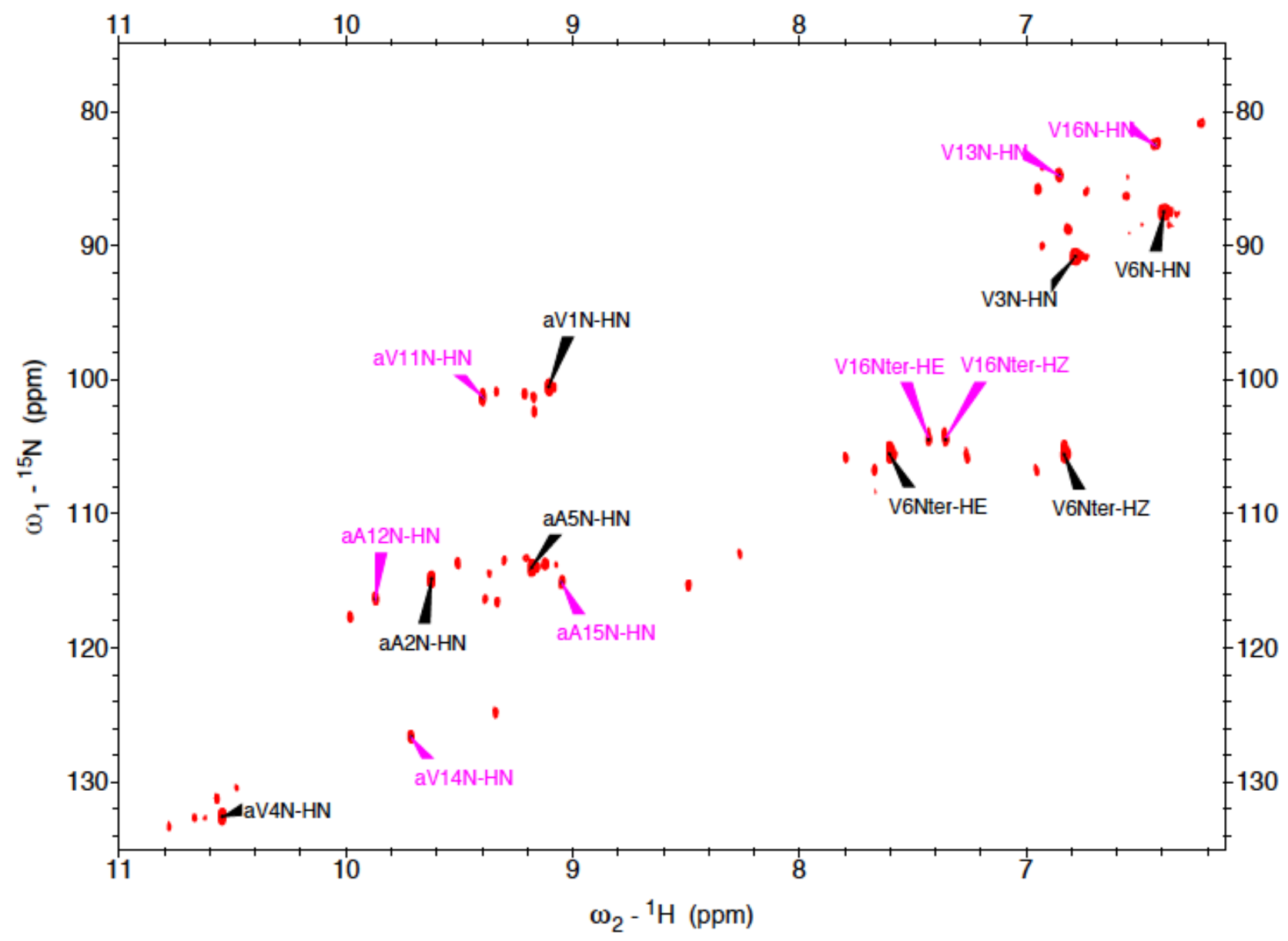

Figure S12: 2D ${ }^{15} \mathrm{~N}-{ }^{1} \mathrm{H}$ HSQC spectrum of compound $5(15 \mathrm{mM})$ in methanol at $273 \mathrm{~K}(500.3 \mathrm{MHz})$. The assignments of the two most-populated conformers are shown in black and magenta (numbered 1-6 and 1116 , respectively). 
A

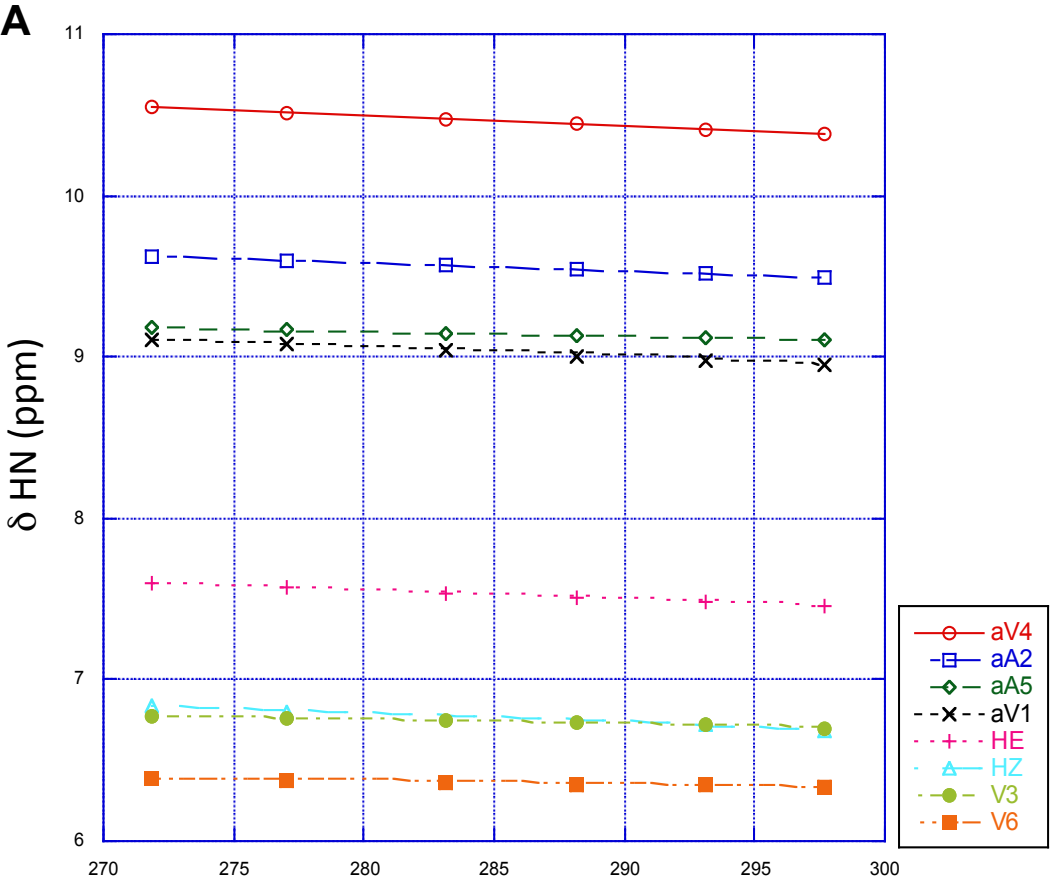

Temperature (K)

B

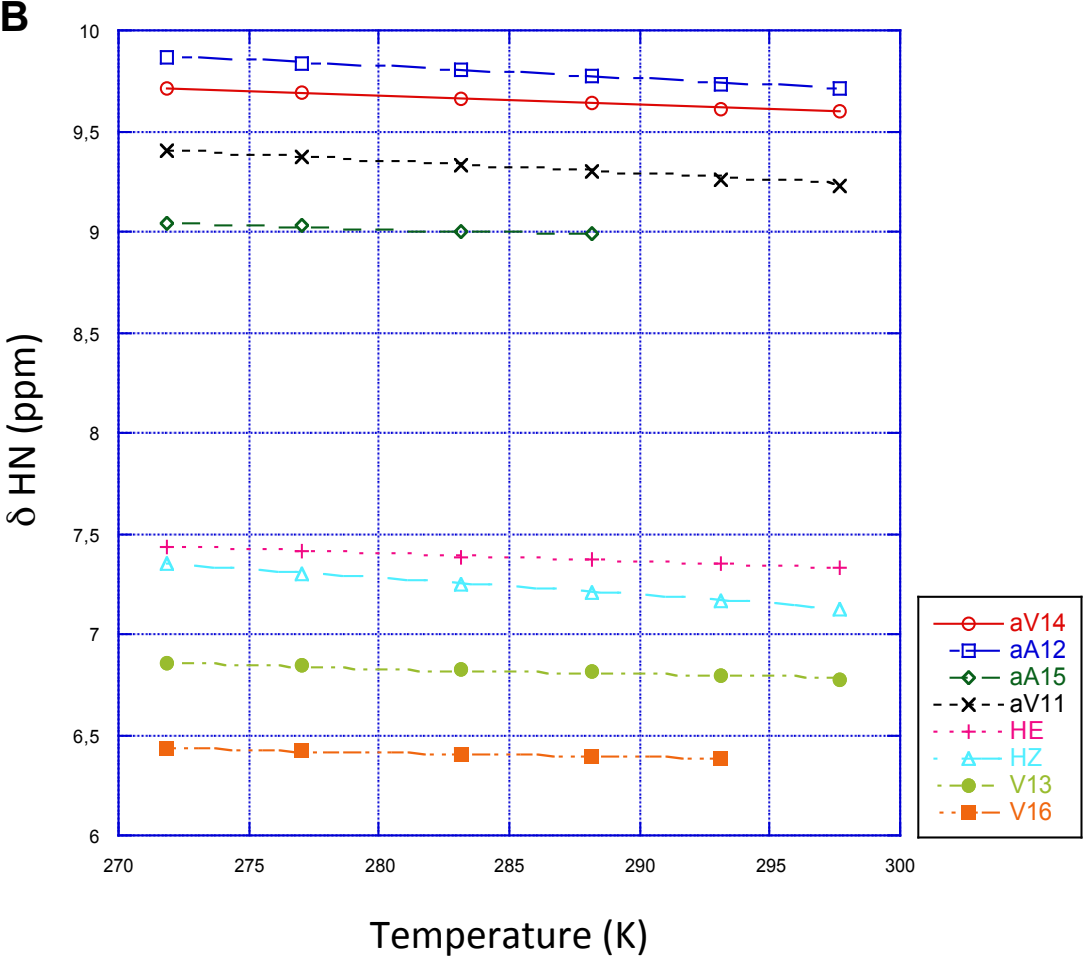

Figure S13: Diagrams of the amide proton temperature coefficients for the two most-populated conformers of compound $\mathbf{5}$ in methanol (5A and $5 B$ ). 


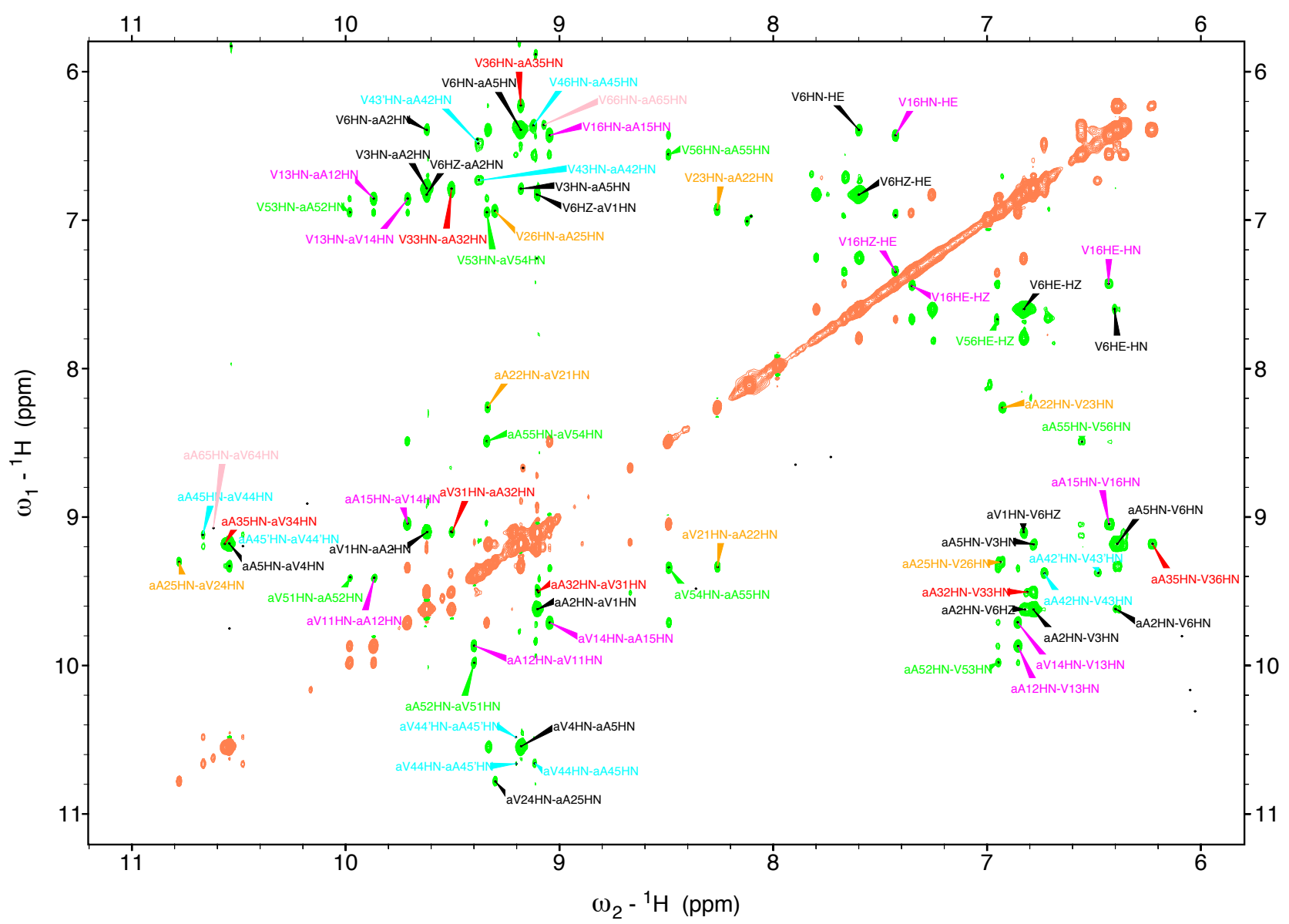

Figure S14: Expansion of the 2D ${ }^{1} \mathrm{H}-{ }^{1} \mathrm{H}$ ROESY spectrum of compound $5(15 \mathrm{mM})$ in methanol at $273 \mathrm{~K}$ (500.3 MHz, mixing time of $500 \mathrm{~ms}$ ), showing the dipolar correlation peaks $\mathrm{NHi}-\mathrm{NHi}+1$ for the 8 detected species. The diagonal and exchange peaks are in orange. 


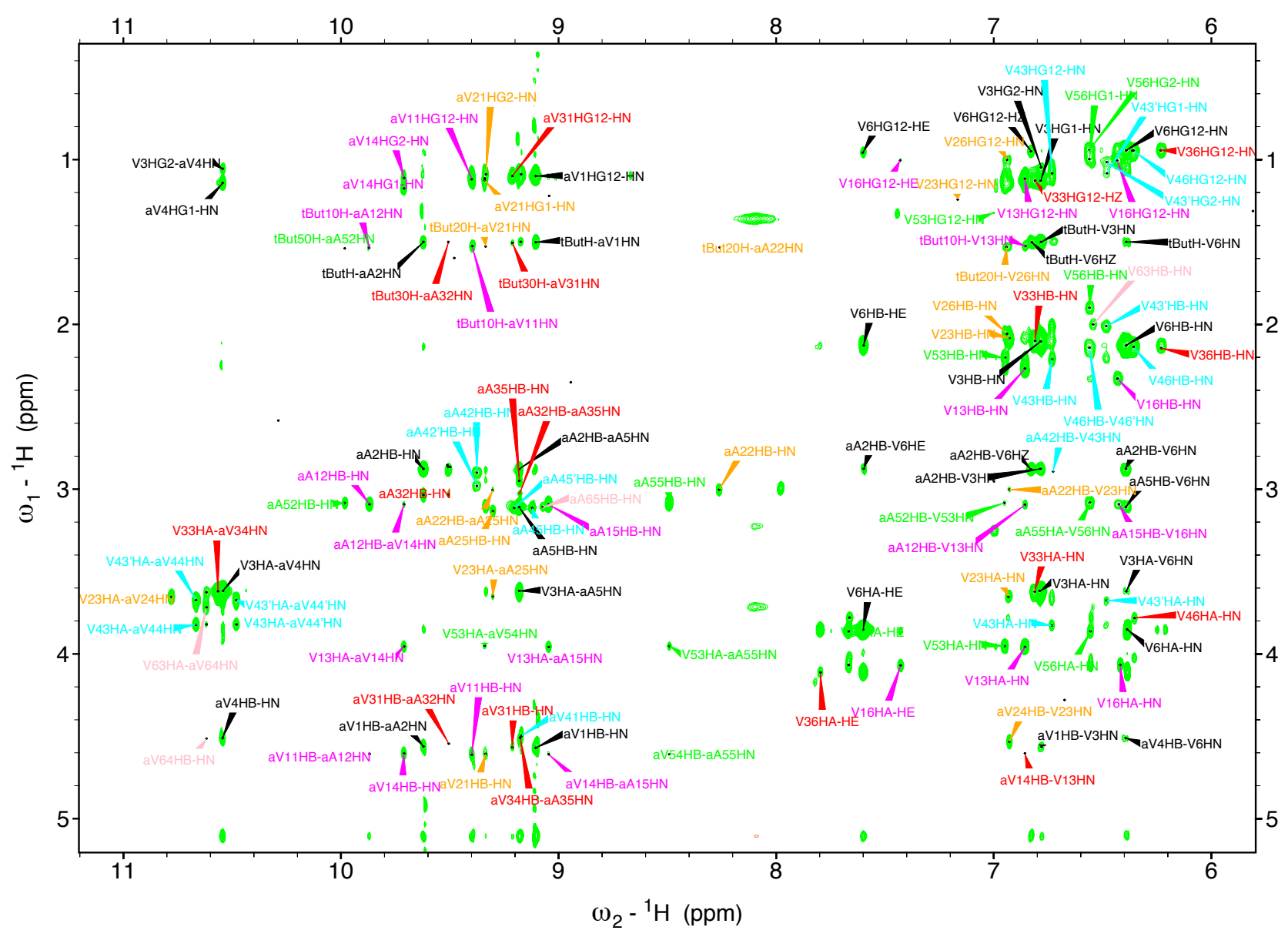

Figure S15: Expansion of the 2D ${ }^{1} \mathrm{H}-{ }^{1} \mathrm{H}$ NMR ROESY spectrum of compound 5 (15 mM) in methanol at $273 \mathrm{~K}(500.3 \mathrm{MHz}$, mixing time of $500 \mathrm{~ms}$ ), showing the long-distance dipolar correlation peaks between $\mathrm{NH}$ and Aliphatic protons for the 8 species. 


\section{X-ray crystallography}

\section{Crystallographic data for Boc-aVal-aAla-Val- $-\mathrm{NH}_{2} 2$}

The crystal structure has been deposited within CCDC with deposition number 1949709.

Formula: $\mathrm{C}_{16} \mathrm{H}_{32} \mathrm{~N}_{6} \mathrm{O}_{5}$

Space group: $\mathrm{P} 2_{1}$

Cell Lengths: a 10.4497(3) b 14.6303(4) c 15.2786(5)

Cell Angles: $\alpha 90 \beta$ 108.211(2) $\gamma 90$

Cell volume : 2218.83

$Z, Z^{\prime}: \mathbf{Z}: 4 \mathbf{Z}^{\prime}: 0$

R-Factor (\%) : 4

A

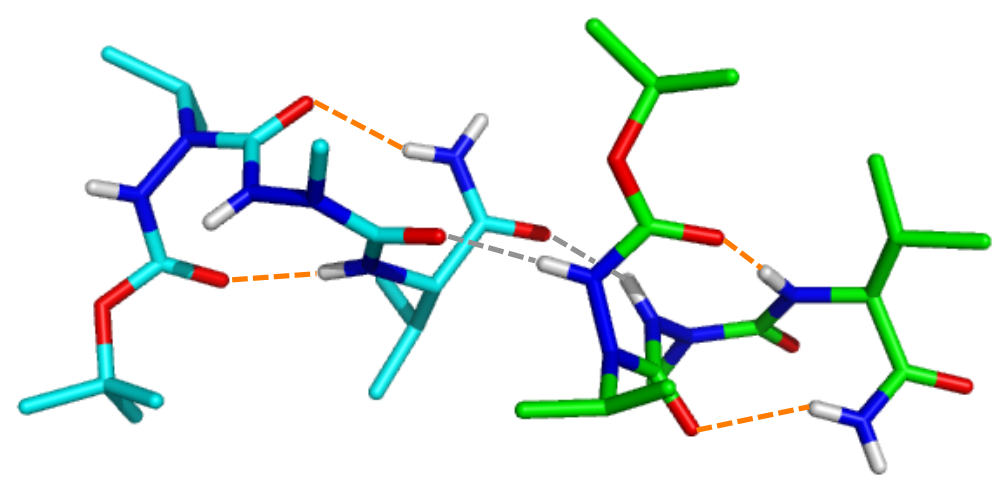

B

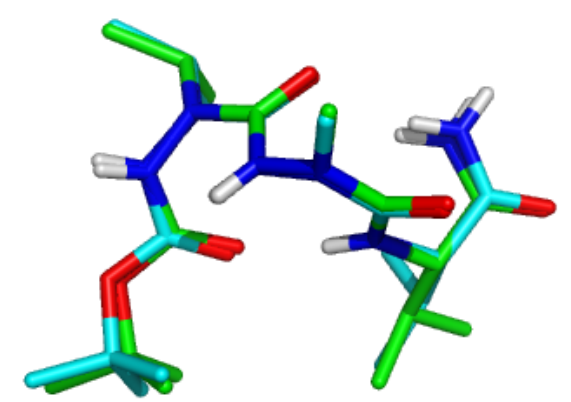

Figure S16: X-ray structure of compound Boc-aVal-aAla-Val- $\mathrm{NH}_{2}$ 2. A) View of the two molecules within the asymmetric unit. Intramolecular hydrogen bonds are displayed in orange and intermolecular hydrogen bonds between the two molecules are displayed in grey. The carbon atoms of the two molecules are shown with different colours, in cyan and green. B) Superimposition of the two molecules of the asymmetric unit. The two chains differ mainly by the rotation of Val3 side chain, with trans $\left(\chi 1+171^{\circ}\right)$ and gauche rotamers $(\chi 1-$ $59^{\circ}$ ). The rms deviation of backbone atoms positions is $0.2 \AA$. 


\section{NMR structures}

\section{conformer A}

A

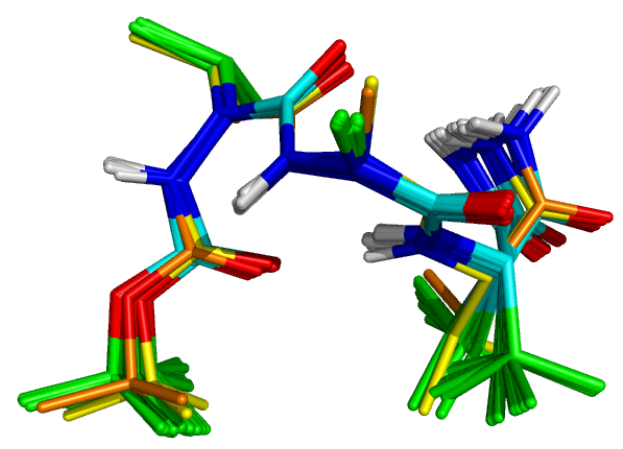

B

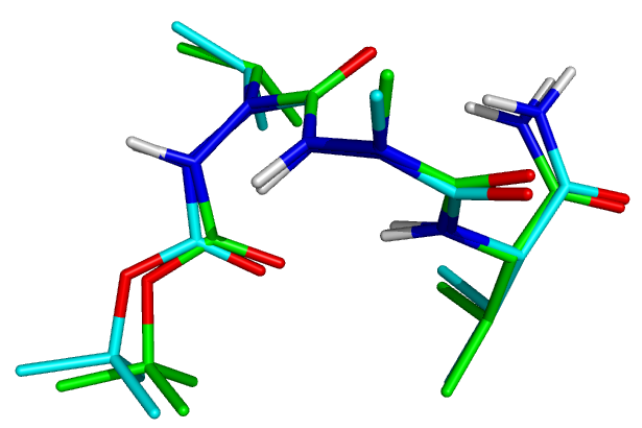

C

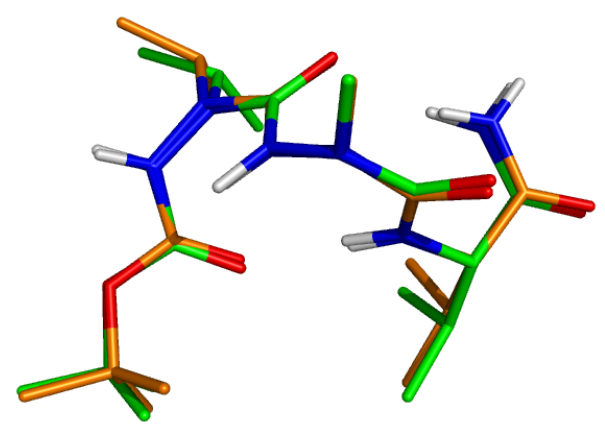

conformer B
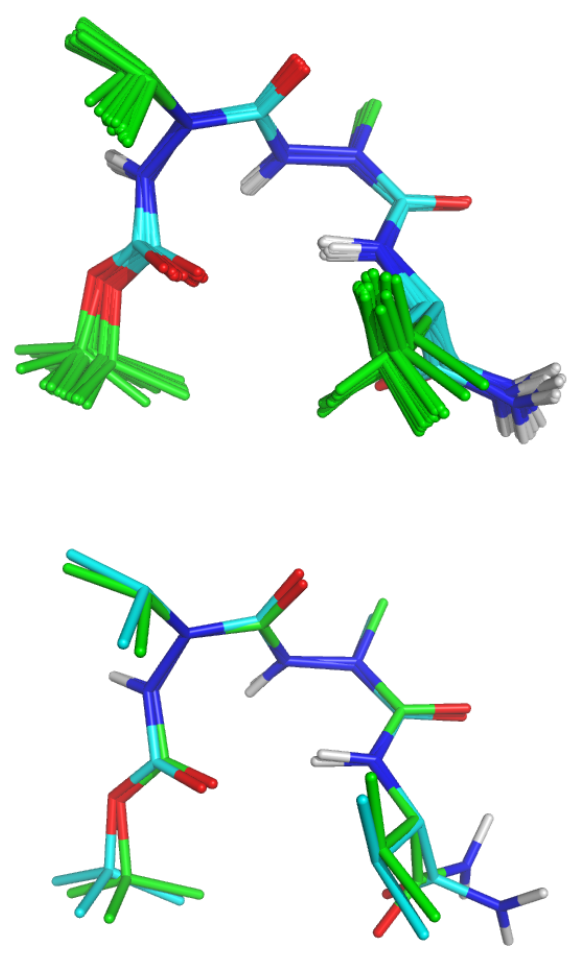

Figure S17: Comparison of the X-ray and NMR structures of compound Boc-aVal-aAla-Val-NH $\mathrm{N}_{2}$ 2. A) View of the two NMR structure ensembles corresponding to conformational isomers $A$ and $B$ observed in solution. In conformer A family, the two X-ray structures of the asymmetric unit are superimposed (carbon atoms coloured in orange and yellow). The carbon atoms of NMR structures are shown in cyan and green colours, corresponding to backbone and side chain atoms, respectively. B) Superimposition of a selected NMR conformer before (cyan carbon atoms) and after DFT refinement (green carbon atoms). C) Superimposition of DFT-refined NMR conformer (green carbon atoms) with the closest X-ray structure (orange carbon atoms). The rms deviation of backbone atoms positions is $0.14 \AA$. 
Table S16. DFT calculations for selected NMR conformers of compound 2. Conformers $2 A-2$ and $2 B$ correspond to the two conformers shown in figures $4 \mathrm{~B}$ and $\mathrm{S} 17$.

\begin{tabular}{|c|c|c|}
\hline Conformer & Energy statistics & Structure \\
\hline $2 A-1$ & $\begin{array}{l}\text { Electronic energy (B3LYP): } \\
-1334.26191331 \mathrm{Ha} \text {. } \\
\text { Lowest frequency: } 21.3604 \mathrm{~cm}^{-1} \text {. } \\
\text { Free energy: }-1333.820938 \mathrm{Ha} \text {. }\end{array}$ & \\
\hline $2 A-2$ & $\begin{array}{l}\text { Electronic energy (B3LYP): } \\
-1334.26179403 \mathrm{Ha} . \\
\text { Lowest frequency: } 19.7783 \mathrm{~cm}^{-1} \text {. } \\
\text { Free energy: }-1333.820796 \mathrm{Ha} \text {. }\end{array}$ & \\
\hline 2B & $\begin{array}{l}\text { Electronic energy (B3LYP): } \\
-1334.25905892 \mathrm{Ha} . \\
\text { Lowest frequency: } 16.2206 \mathrm{~cm}^{-1} \text {. } \\
\text { Free energy: }-1333.820185 \mathrm{Ha} \text {. }\end{array}$ & \\
\hline
\end{tabular}




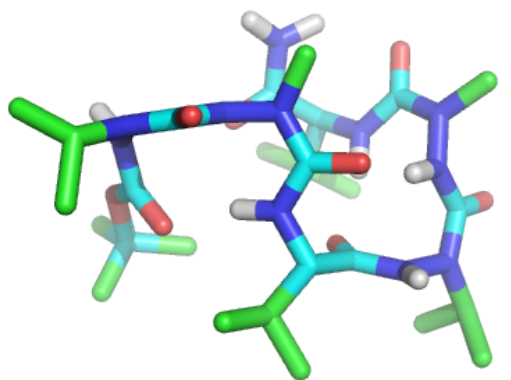

$+/+/+/+/-$

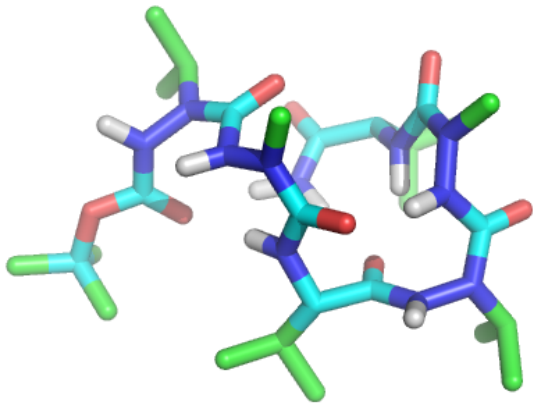

$-/-I-I+/+1-$

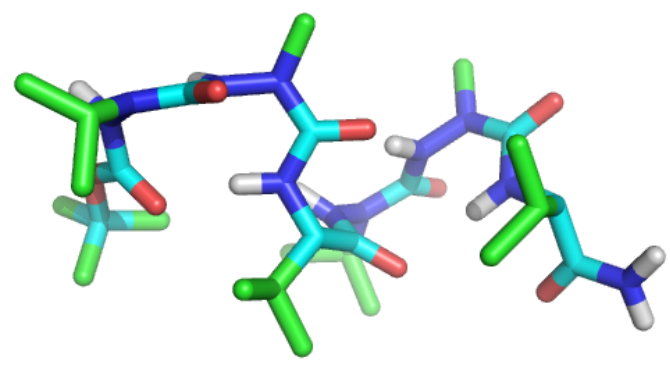

$+1+1-1-1-1-$

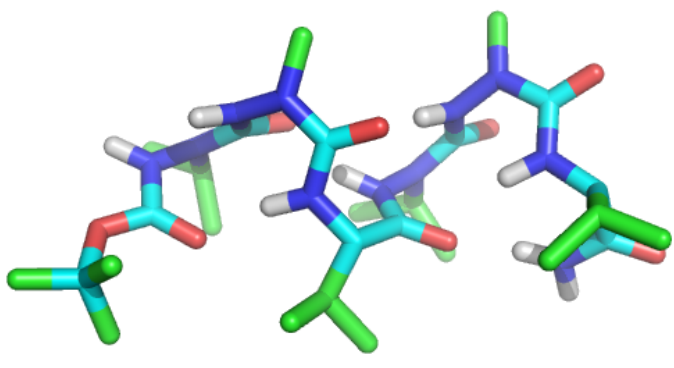

$-1-1-1-1-$

Figure S18. Modelled conformations of Boc-aVal-aAla-Val-aVal-aAla-Val- $\mathrm{NH}_{2} \mathbf{5}$ showing repeated $\beta$-turns or helical conformations. Structures were calculated using AMBER and refined by DFT at the B3LYP-D3/ccpVTZ level with methanol PCM solvation model (see Table S18). The conformers differ by the sign of $\phi$ dihedral angles within each diazapeptide unit (either $+/+$ or $-/-$ ). 
Ab initio conformational search: molecular mechanics studies and DFT calculations

\begin{tabular}{|c|c|c|c|c|c|c|c|c|c|c|c|c|}
\hline \multirow{2}{*}{$\begin{array}{l}\text { Cpd. / } \\
\text { conf. }\end{array}$} & \multicolumn{3}{|c|}{ Peptide bond } & \multicolumn{2}{|c|}{$\begin{array}{c}\text { Urea } \\
\text { geometry }\end{array}$} & \multicolumn{3}{|c|}{$\varphi$ angle $\left({ }^{\circ}\right)$} & \multicolumn{3}{|c|}{$\psi$ angle $\left({ }^{\circ}\right)$} & \multirow{2}{*}{$\begin{array}{c}\Delta \mathrm{G} \\
\left.\text { (kcal mol }^{-1}\right)\end{array}$} \\
\hline & 1 & 2 & 3 & 1 & 2 & 1 & 2 & 3 & 1 & 2 & 3 & \\
\hline $1(\mathrm{a})$ & trans & trans & trans & $n / a$ & $\mathrm{n} / \mathrm{a}$ & -66.8 & -58.6 & -110.1 & -17.3 & -23.8 & 13.8 & 0.00 \\
\hline 1 (b) & trans & trans & trans & $n / a$ & $n / a$ & -131.0 & -152.7 & -131.8 & 163.2 & 157.0 & 134.4 & 3.86 \\
\hline 1 (c) & trans & trans & trans & $\mathrm{n} / \mathrm{a}$ & $\mathrm{n} / \mathrm{a}$ & -131.7 & -64.2 & -134.4 & 162.1 & 139.3 & 131.9 & 3.87 \\
\hline $1(\mathrm{~d})$ & trans & trans & trans & $\mathrm{n} / \mathrm{a}$ & $\mathrm{n} / \mathrm{a}$ & -60.1 & 58.4 & 59.9 & 129.2 & 24.6 & 35.1 & 4.90 \\
\hline $2(a)$ & trans & trans & trans & $E, Z$ & $E, Z$ & -70.7 & -67.3 & -105.4 & -13.2 & -17.2 & 11.8 & 0.00 \\
\hline 2 (b) & trans & trans & trans & $E, Z$ & $E, Z$ & 72.6 & 80.0 & -124.7 & 12.2 & 12.3 & 9.8 & 0.70 \\
\hline $3(\mathrm{a})$ & trans & trans & trans & $E, Z$ & $E, Z$ & 72.4 & 80.3 & -59.2 & 12.9 & 2.7 & -38.9 & 0.00 \\
\hline $3(\mathrm{~b})$ & trans & trans & trans & $E, Z$ & $E, Z$ & -80.8 & -73.4 & -94.1 & -6.5 & -14.2 & -56.2 & 2.03 \\
\hline 3 (c) & trans & trans & trans & $E, Z$ & $E, Z$ & $-101,7$ & 91.4 & -94.9 & 0.8 & 2.7 & 0.4 & 2.40 \\
\hline 4 (a) & trans & trans & trans & $E, Z$ & $E, Z$ & 89.9 & -70.9 & -80.2 & 18.0 & -20.2 & -7.4 & 0.00 \\
\hline $4(b)$ & trans & cis & trans & $Z, E$ & $E, Z$ & 54.5 & 107.2 & -86.7 & -149.3 & -29.3 & -13.1 & 1.81 \\
\hline 4 (c) & cis & trans & trans & $Z, Z$ & $E, Z$ & -82.0 & 85.7 & -120.0 & -180.0 & 20.9 & 6.0 & 2.71 \\
\hline 4 (d) & cis & trans & trans & $Z, Z$ & $Z, Z$ & 61.1 & -65.0 & -125.1 & -172.3 & 163.9 & 9.7 & 3.95 \\
\hline $4(\mathrm{e})$ & trans & trans & trans & $Z, Z$ & $Z, Z$ & -62.7 & -63.3 & -122.6 & 164.3 & 165.9 & 7.8 & 4.18 \\
\hline
\end{tabular}




\begin{tabular}{|c|c|c|}
\hline Conformer & Energy statistics & Structure \\
\hline $1 a$ & $\begin{array}{l}\text { Electronic energy (B3LYP): } \\
-1302.22855290 \mathrm{Ha} \text {. } \\
\text { Lowest frequency: } 16.2871 \mathrm{~cm}^{-1} \text {. } \\
\text { Free energy: }-1301.763619 \mathrm{Ha} .\end{array}$ & \\
\hline $1 b$ & $\begin{array}{l}\text { Electronic energy (B3LYP): } \\
\text {-1302.21580581 Ha. } \\
\text { Lowest frequency: } 9.9099 \mathrm{~cm}^{-1} \text {.Free } \\
\text { energy: }-1301.75747 \mathrm{Ha} .\end{array}$ & \\
\hline $1 c$ & $\begin{array}{l}\text { Electronic energy (B3LYP): } \\
-1302.21686165 \mathrm{Ha} \text {. } \\
\text { Lowest frequency: } 9.8326 \mathrm{~cm}^{-1} \text {. } \\
\text { Free energy: }-1301.757458 \mathrm{Ha} .\end{array}$ & \\
\hline $1 d$ & $\begin{array}{l}\text { Electronic energy (B3LYP): } \\
-1302.22029291 \mathrm{Ha} . \\
\text { Lowest frequency: } 14.2234 \mathrm{~cm}^{-1} \text {. } \\
\text { Free energy: }-1301.755818 \mathrm{Ha} \text {. }\end{array}$ & \\
\hline $2 a$ & $\begin{array}{l}\text { Electronic energy (B3LYP): } \\
-1334.26277825 \mathrm{Ha} . \\
\text { Lowest frequency: } 19.9132 \mathrm{~cm}^{-1} \text {. } \\
\text { Free energy: }-1333.822029 \mathrm{Ha} \text {. }\end{array}$ & \\
\hline
\end{tabular}




\begin{tabular}{|c|c|}
\hline $2 b$ & $\begin{array}{l}\text { Electronic energy (B3LYP): } \\
-1334.26046535 \mathrm{Ha} . \\
\text { Lowest frequency: } 24.5349 \mathrm{~cm}^{-1} \text {. } \\
\text { Free energy: }-1333.820911 \mathrm{Ha} \text {. }\end{array}$ \\
\hline $3 a$ & $\begin{array}{l}\text { Electronic energy (B3LYP): } \\
\text {-1624.59308144 Ha. } \\
\text { Lowest frequency: } 17.4456 \mathrm{~cm}^{-1} \text {. } \\
\text { Free energy: }-1624.064872 \mathrm{Ha} \text {. }\end{array}$ \\
\hline $3 b$ & $\begin{array}{l}\text { Electronic energy (B3LYP): } \\
-1624.59053947 \mathrm{Ha} . \\
\text { Lowest frequency: } 14.2055 \mathrm{~cm}^{-1} \text {. } \\
\text { Free energy: }-1624.061572 \mathrm{Ha} \text {. }\end{array}$ \\
\hline $3 c$ & $\begin{array}{l}\text { Electronic energy (B3LYP): } \\
-1624.58678978 \mathrm{Ha} . \\
\text { Lowest frequency: } 12.9646 \mathrm{~cm}^{-1} \text {. } \\
\text { Free energy: }-1624.061055 \mathrm{Ha} \text {. }\end{array}$ \\
\hline $4 a$ & $\begin{array}{l}\text { Electronic energy (B3LYP): } \\
-1176.94113258 \mathrm{Ha} . \\
\text { Lowest frequency: } 13.7268 \mathrm{~cm}^{-1} \text {. } \\
\text { Free energy: }-1176.608668 \mathrm{Ha} \text {. }\end{array}$ \\
\hline $4 b$ & $\begin{array}{l}\text { Electronic energy (B3LYP): } \\
-1176.94083349 \mathrm{Ha} . \\
\text { Lowest frequency: } 19.3529 \mathrm{~cm}^{-1} \text {. } \\
\text { Free energy: }-1176.605776 \mathrm{Ha} \text {. }\end{array}$ \\
\hline
\end{tabular}




\begin{tabular}{|l|l|}
\hline $4 \mathbf{c}$ & $\begin{array}{l}\text { Electronic energy (B3LYP): } \\
-1176.93661645 \mathrm{Ha} . \\
\text { Lowest frequency: } 12.4244 \mathrm{~cm}^{-1} . \\
\text { Free energy: }-1176.604347 \mathrm{Ha} .\end{array}$ \\
$\mathbf{4 d}$ & $\begin{array}{l}\text { Electronic energy (B3LYP): } \\
-1176.93453150 \mathrm{Ha} . \\
\text { Lowest frequency: } 8.8321 \mathrm{~cm}^{-1} . \\
\text { Free energy: }-1176.602374 \mathrm{Ha}^{\prime}\end{array}$ \\
$\mathbf{4 e}$ & $\begin{array}{l}\text { Electronic energy (B3LYP): } \\
-1176.93232000 \mathrm{Ha} . \\
\text { Lowest frequency: } 10.2437 \mathrm{~cm}^{-1} . \\
\text { Free energy: }-1176.60201 \mathrm{Ha}^{2}\end{array}$ \\
&
\end{tabular}




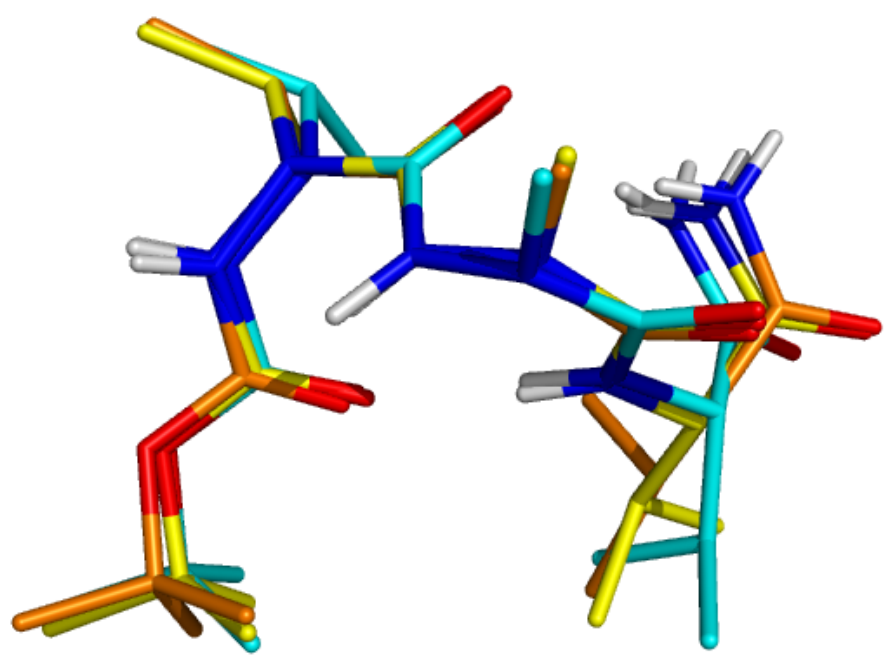

Figure S19: Comparison of conformer 2a calculated from ab initio conformational search with the X-ray structure of compound Boc-aVal-aAla-Val- $\mathrm{NH}_{2}$ 2. Carbon atoms of conformer $\mathbf{2 a}$ are shown in cyan while carbon atoms of the two crystallographic structures within the asymmetric unit are shown in orange and yellow. The rms deviation of backbone atoms positions between $\mathbf{2 a}$ and the closest $\mathrm{X}$-ray conformer is $0.27 \AA$. 


\section{Atomic coordinates of molecular models}

\section{Compound 1, conformation a (from ab initio conformational search)}

\begin{tabular}{|c|c|c|c|}
\hline C & -1.773474 & 3.355175 & -0.187459 \\
\hline O & -2.432826 & 2.048733 & 0.044483 \\
\hline C & -2.939759 & 4.237741 & -0.615079 \\
\hline C & -0.758977 & 3.210707 & -1.317593 \\
\hline C & -1.151973 & 3.869844 & 1.106894 \\
\hline C & -1.737192 & 0.958117 & 0.384448 \\
\hline $\mathrm{N}$ & -2.502506 & -0.157851 & 0.258629 \\
\hline 0 & -0.567186 & 0.925519 & 0.7 \\
\hline C & -2.032105 & -1.448456 & 0.729 \\
\hline $\mathrm{C}$ & -0.837161 & -1.976733 & -0.06 \\
\hline $\mathrm{C}$ & -3.163400 & -2.498552 & 0.7 \\
\hline $\mathrm{N}$ & -0.5 & -1.422193 & $-1 \cdot 2$ \\
\hline $\mathrm{O}$ & -0.1 & -2.859 & 0.4 \\
\hline C & 65672 & -1.754 & -2. \\
\hline $\mathrm{C}$ & 04217 & -1.481 & -1 \\
\hline $\mathrm{C}$ & 0.5 & -0.99 & $-3 \cdot 3$ \\
\hline $\mathrm{N}$ & 1.9 & -0.562773 & -0.3 \\
\hline O & 2.9 & -2.085 & -1.7 \\
\hline C & 3.1 & -0.272 & \\
\hline $\mathrm{C}$ & 3.0 & -0.763477 & 1.8 \\
\hline C & 3.5 & 1.205480 & 0.3 \\
\hline $\mathrm{N}$ & & -1.62 & 2. \\
\hline 0 & 3.8 & -0.399059 & 2.6 \\
\hline C & -4.2 & -2.120972 & 1.7 \\
\hline C & -3.7 & -2.772244 & -0.6 \\
\hline C & 3.8 & 558 & -1.1 \\
\hline $\mathrm{C}$ & 2.566 & 2.157038 & 0.9 \\
\hline $\mathrm{H}$ & -3.6 & 967 & 0 . \\
\hline $\mathrm{H}$ & -2.5 & 021 & $-0 . \varepsilon$ \\
\hline $\mathrm{H}$ & -3.4 & 3.84 & -1.5 \\
\hline $\mathrm{H}$ & 0.0 & 265 & -1 \\
\hline $\mathrm{H}$ & $-1 \cdot 2$ & 489 & -2.2 \\
\hline $\mathrm{H}$ & -0.3 & 4.190623 & -1.5 \\
\hline $\mathrm{H}$ & -0.3 & 3.252869 & 1 \\
\hline $\mathrm{H}$ & -0.7 & 450 & \\
\hline $\mathrm{H}$ & $-1 \cdot 8$ & 942 & 1. \\
\hline $\mathrm{H}$ & -3.463 & -0.036979 & -0.0 \\
\hline $\mathrm{H}$ & $-1 \cdot 6$ & -1.33 & 1. \\
\hline $\mathrm{H}$ & -2.67 & -3.41 & 1.1 \\
\hline $\mathrm{H}$ & -1.226 & -0.70 & -1.5 \\
\hline $\mathrm{H}$ & 0.57 & -2.8 & -2.2 \\
\hline $\mathrm{H}$ & 1.386 & -1.247636 & -3.9 \\
\hline $\mathrm{H}$ & -0.381 & -1.262 & -3.9 \\
\hline $\mathrm{H}$ & 0.514 & 0.082 & -3.2 \\
\hline $\mathrm{H}$ & 1.052 & -0.0 & -0.1 \\
\hline $\mathrm{H}$ & 3.903 & -0.875803 & -0.0 \\
\hline $\mathrm{H}$ & 4.497 & 1.254519 & 0.8 \\
\hline $\mathrm{H}$ & 1.38 & -1.985 & \\
\hline $\mathrm{H}$ & 2.017 & -2.003143 & 3.079 \\
\hline $\mathrm{H}$ & -4.966 & -2.922662 & 1.8 \\
\hline $\mathrm{H}$ & -3.793 & -1.9 & \\
\hline $\mathrm{H}$ & -4.772 & -1.216609 & 1.508087 \\
\hline $\mathrm{H}$ & -4.505 & -3.576820 & -0.536911 \\
\hline $\mathrm{H}$ & -4.293291 & -1.898267 & -1.0075 \\
\hline $\mathrm{H}$ & -3.017595 & -3.070950 & -1.336195 \\
\hline $\mathrm{H}$ & 4.190742 & 2.621960 & -1.203105 \\
\hline $\mathrm{H}$ & 4.522514 & 0.939437 & -1.6283 \\
\hline $\mathrm{H}$ & 2.877648 & 1.569586 & -1.7292 \\
\hline $\mathrm{H}$ & 2.430877 & 1.906582 & 2.0362 \\
\hline $\mathrm{H}$ & 2.931 & & \\
\hline $\mathrm{H}$ & 1.5 & & \\
\hline
\end{tabular}




\section{Compound 1, conformation $\mathbf{b}$ (from ab initio conformational search)}

\begin{tabular}{|c|c|c|c|}
\hline $\mathrm{C}$ & 6.599092 & -0.727395 & -0.049562 \\
\hline 0 & 5.242545 & -0.416415 & -0.534189 \\
\hline $\mathrm{C}$ & 6.549470 & -1.892873 & 0.934694 \\
\hline C & 7.237265 & 0.520237 & 0.555290 \\
\hline $\mathrm{C}$ & 7.320647 & -1.134198 & -1.330047 \\
\hline C & 4.269903 & 0.011373 & 0.297461 \\
\hline $\mathrm{N}$ & 3.147492 & 0.277953 & -0.416782 \\
\hline O & 4.375099 & 0.155460 & \\
\hline $\mathrm{C}$ & 1.878720 & 0.587435 & \\
\hline C & 0.795471 & -0.284573 & -0 . \\
\hline $\mathrm{C}$ & 1.505882 & 2.09 & \\
\hline $\mathrm{N}$ & -0.350673 & -0.37 & \\
\hline O & 0.961682 & -0.81 & -1 \\
\hline $\mathrm{C}$ & -1.53 & -1.0 & -0 . \\
\hline C & -2.75 & -0.3 & \\
\hline C & -1.50 & -2.5 & \\
\hline $\mathrm{N}$ & -3.9 & -0.5 & -0 . \\
\hline 0 & -2.656 & 0.1 & \\
\hline $\mathrm{C}$ & -5.203290 & -0.1 & \\
\hline C & -6.163962 & -1.2 & \\
\hline $\mathrm{C}$ & -5.717564 & 1.1 & -0 \\
\hline $\mathrm{N}$ & -6.965994 & -1.59 & \\
\hline $\mathrm{O}$ & -6.18 & -1.88 & -1 \\
\hline C & 823 & 2.9 & \\
\hline $\mathrm{C}$ & 1.36 & 2.5 & -1 \\
\hline C & -7.10 & 1.5 & \\
\hline C & -4.7 & 2.3 & -0 \\
\hline $\mathrm{H}$ & 924 & -2.20 & \\
\hline $\mathrm{H}$ & 6. & -1.6 & \\
\hline $\mathrm{H}$ & 6.0 & -2.7 & \\
\hline $\mathrm{H}$ & 7.1 & 1.3 & -0 \\
\hline $\mathrm{H}$ & 6.7 & 0.8 & \\
\hline $\mathrm{H}$ & 8.28 & 0.316 & \\
\hline $\mathrm{H}$ & 8.3 & -1.394153 & -1 \\
\hline $\mathrm{H}$ & 6.8 & -1.9 & -1 \\
\hline $\mathrm{H}$ & 7.31 & -0.3 & -2 \\
\hline $\mathrm{H}$ & 3.1 & -0.03 & -1 \\
\hline $\mathrm{H}$ & 1.9 & 0.33 & 1.2 \\
\hline $\mathrm{H}$ & 0.53 & 2.2 & \\
\hline $\mathrm{H}$ & -0.449 & 0.0 & \\
\hline $\mathrm{H}$ & -1.572677 & -0.894893 & $-1 \cdot 3$ \\
\hline $\mathrm{H}$ & -2.393571 & -3.0 & \\
\hline $\mathrm{H}$ & -1.474443 & -2.720523 & \\
\hline $\mathrm{H}$ & -0.624920 & -2.994000 & -0.4 \\
\hline $\mathrm{H}$ & -3.911446 & -1.03 & -1 \\
\hline $\mathrm{H}$ & -5.083706 & 0.114 & \\
\hline $\mathrm{H}$ & -5.791930 & 0.885213 & -1.5 \\
\hline $\mathrm{H}$ & -7.646540 & -2.333647 & 0 . \\
\hline $\mathrm{H}$ & -6.935514 & -1.10 & \\
\hline $\mathrm{H}$ & 2.229055 & 4.001279 & 0.8 \\
\hline $\mathrm{H}$ & 2.603752 & 2.650496 & 1.8 \\
\hline $\mathrm{H}$ & 3.511930 & 2.864291 & \\
\hline $\mathrm{H}$ & 1.084454 & 3.600337 & -1.3946 \\
\hline $\mathrm{H}$ & 2.310436 & 2.433106 & -1.8 \\
\hline $\mathrm{H}$ & 0.604834 & 1.976685 & \\
\hline $\mathrm{H}$ & -7.439088 & 2.459189 & -0.52178 \\
\hline $\mathrm{H}$ & -7.851520 & 0.785315 & -0.16322 \\
\hline $\mathrm{H}$ & -7.080185 & 1.788139 & \\
\hline $\mathrm{H}$ & -5.077433 & 3.174139 & -0.9129 \\
\hline $\mathrm{H}$ & -4.626678 & 2.599438 & 0.6947 \\
\hline $\mathrm{H}$ & -3.738142 & 2.043524 & -0.725 \\
\hline
\end{tabular}




\section{Compound 1, conformation c (from ab initio conformational search)}

\begin{tabular}{|c|c|c|c|}
\hline C & -5.823278 & 1.235036 & 0.664272 \\
\hline O & -4.394478 & 1.061069 & 0.347199 \\
\hline C & -6.157864 & 0.535685 & 1.979024 \\
\hline C & -6.680316 & 0.736453 & -0.496092 \\
\hline $\mathrm{C}$ & -5.945546 & 2.747852 & 0.813070 \\
\hline $\mathrm{C}$ & -3.859271 & -0.156003 & 0.117943 \\
\hline N & -2.548920 & -0.021359 & -0.206997 \\
\hline O & -4.453761 & -1.219875 & 0.176121 \\
\hline $\mathrm{C}$ & -1.656304 & -1.151467 & -0.344988 \\
\hline $\mathrm{C}$ & -0.378994 & -0.855865 & 0.445198 \\
\hline C & -1.309221 & -1.470244 & -1.827604 \\
\hline $\mathrm{N}$ & 0.387765 & -1.925202 & 0.736466 \\
\hline O & -0.055841 & 0.288827 & 0.753327 \\
\hline C & 1.732943 & -1.769298 & 1.263199 \\
\hline C & 2.624291 & -1.076189 & 0.216121 \\
\hline C & 2.318837 & -3.133895 & 1.616108 \\
\hline $\mathrm{N}$ & 3.470190 & -0.155963 & 0.71 \\
\hline O & 2.568338 & -1.388760 & -0.968356 \\
\hline C & 4.428169 & 0.587893 & -0.088284 \\
\hline $\mathrm{C}$ & 5.757808 & 0.561236 & 0.67 \\
\hline C & 3.969117 & 2.047167 & -0.32 \\
\hline $\mathrm{N}$ & 6.847689 & 0.230595 & -0.041862 \\
\hline O & 5.793986 & 0.844067 & 1.865184 \\
\hline C & -2.573000 & -1.845771 & -2.60 \\
\hline $\mathrm{C}$ & -0.558392 & -0.320763 & -2.503181 \\
\hline C & 5.016476 & 2.836467 & -1.118893 \\
\hline C & 2.613535 & 2.081973 & -1.03 \\
\hline $\mathrm{H}$ & -5.482690 & 0.872983 & 2.765940 \\
\hline $\mathrm{H}$ & -7.177485 & 0.787345 & 2.27 \\
\hline $\mathrm{H}$ & -6.076316 & -0.542883 & 1.881609 \\
\hline $\mathrm{H}$ & -6.599711 & -0.340734 & -0.609146 \\
\hline $\mathrm{H}$ & -6.369358 & 1.214852 & -1.425227 \\
\hline $\mathrm{H}$ & -7.723305 & 0.994029 & -0.310605 \\
\hline $\mathrm{H}$ & -6.974333 & 3.014557 & 1.052762 \\
\hline $\mathrm{H}$ & -5.297907 & 3.106434 & 1.612850 \\
\hline $\mathrm{H}$ & -5.663596 & 3.246907 & -0.113788 \\
\hline $\mathrm{H}$ & -2.117829 & 0.878734 & -0.067650 \\
\hline $\mathrm{H}$ & -2.153994 & -2.021758 & 0.082192 \\
\hline $\mathrm{H}$ & -0.650418 & -2.342145 & -1.809061 \\
\hline $\mathrm{H}$ & 0.116993 & -2.830900 & 0.390645 \\
\hline $\mathrm{H}$ & 1.674792 & -1.147120 & 2.156241 \\
\hline $\mathrm{H}$ & 3.315940 & -3.017354 & 2.036972 \\
\hline $\mathrm{H}$ & 1.689890 & -3.636787 & 2.349827 \\
\hline $\mathrm{H}$ & 2.391839 & -3.758097 & 0.724913 \\
\hline $\mathrm{H}$ & 3.444111 & 0.067953 & 1.695883 \\
\hline $\mathrm{H}$ & 4.511481 & 0.075772 & -1.045392 \\
\hline $\mathrm{H}$ & 3.860804 & 2.504324 & 0.658931 \\
\hline $\mathrm{H}$ & 6.796768 & -0.002552 & -1.017623 \\
\hline $\mathrm{H}$ & 7.751313 & 0.239261 & 0.402532 \\
\hline $\mathrm{H}$ & -2.318457 & -2.125833 & -3.624799 \\
\hline $\mathrm{H}$ & -3.093869 & -2.682128 & -2.135448 \\
\hline $\mathrm{H}$ & -3.264038 & -1.002856 & -2.646402 \\
\hline $\mathrm{H}$ & -0.352731 & -0.569137 & -3.544961 \\
\hline $\mathrm{H}$ & -1.157133 & 0.591957 & -2.491280 \\
\hline $\mathrm{H}$ & 0.392892 & -0.117364 & -2.013052 \\
\hline $\mathrm{H}$ & 5.191209 & 2.377842 & -2.095093 \\
\hline $\mathrm{H}$ & 4.667707 & 3.855298 & -1.288407 \\
\hline $\mathrm{H}$ & 5.972145 & 2.894308 & -0.598367 \\
\hline $\mathrm{H}$ & 2.280881 & 3.114359 & -1.154253 \\
\hline $\mathrm{H}$ & 2.688849 & 1.639585 & -2.033559 \\
\hline $\mathrm{H}$ & 1.848594 & 1.540423 & -0.484259 \\
\hline
\end{tabular}




\section{Compound 1, conformation d (from ab initio conformational search)}

\begin{tabular}{|c|c|c|c|}
\hline C & 0.539766 & 3.805571 & -0.055281 \\
\hline 0 & 1.456418 & 2.718258 & 0.346440 \\
\hline C & 0.879379 & 4.903463 & 0.945799 \\
\hline C & -0.913548 & 3.367928 & 0.094628 \\
\hline $\mathrm{C}$ & 0.869658 & 4.238440 & -1.479714 \\
\hline C & 1.486667 & 1.534513 & -0.282186 \\
\hline $\mathrm{N}$ & 2.537380 & 0.798490 & \\
\hline 0 & 0.696391 & 1.147782 & -1.135332 \\
\hline C & 2.750656 & -0.565286 & -0.293235 \\
\hline $\mathrm{C}$ & 1.539384 & -1.419693 & \\
\hline C & 4.045642 & -1.135158 & \\
\hline $\mathrm{N}$ & 0.997238 & -2.155945 & -0 . \\
\hline 0 & 1.096538 & -1.403224 & \\
\hline C & -0.161 & -3.026258 & -0.7 \\
\hline C & $-1 \cdot 39$ & -2.263446 & -0.1 \\
\hline $\mathrm{C}$ & 0.15 & -4.238683 & 0 \\
\hline $\mathrm{N}$ & -1.45 & -0.946060 & -0 . \\
\hline O & -2.30 & -2.856980 & 0 \\
\hline $\mathrm{C}$ & -2.54 & -0.096208 & -0 \\
\hline C & -2.642632 & -0.097860 & 1.5 \\
\hline C & -3.90 & -0.34 & -0 . \\
\hline $\mathrm{N}$ & -1.467388 & -0.177537 & \\
\hline O & -3.712741 & 0.047412 & 2 \\
\hline C & 4.223 & -2.61 & -0 . \\
\hline C & 5.26 & -0.325808 & -0 \\
\hline C & -4.757497 & 0.925351 & -0.6 \\
\hline C & -3.730063 & -0.804323 & -2.1 \\
\hline $\mathrm{H}$ & 1.9 & 5 & \\
\hline $\mathrm{H}$ & 0.28 & 5.7 & 0 . \\
\hline $\mathrm{H}$ & 0.6 & 4.57 & 1 . \\
\hline $\mathrm{H}$ & -1.193691 & 2.675 & -0 . \\
\hline $\mathrm{H}$ & -1.068 & 2.890 & 1.0 \\
\hline $\mathrm{H}$ & -1.56 & 4.243 & \\
\hline $\mathrm{H}$ & 0.66 & 3.43 & -2.1 \\
\hline $\mathrm{H}$ & 0.261 & 5.103 & -1.7 \\
\hline $\mathrm{H}$ & 1.92 & 4.519 & -1.5 \\
\hline $\mathrm{H}$ & 3.0 & 1.1 & \\
\hline $\mathrm{H}$ & 2.85 & -0.559 & $-1 \cdot 3$ \\
\hline $\mathrm{H}$ & 3.940 & -1.056101 & 1.4 \\
\hline $\mathrm{H}$ & 1.392155 & -2.085 & -1.8 \\
\hline $\mathrm{H}$ & -0.432106 & -3.373386 & -1.7 \\
\hline $\mathrm{H}$ & -0.722942 & -4.874199 & 0.2 \\
\hline $\mathrm{H}$ & 0.9666 & -4.80 & -0.2 \\
\hline $\mathrm{H}$ & 0.452259 & -3.920330 & 1.167 \\
\hline $\mathrm{H}$ & -0.664325 & -0.487647 & -0.901063 \\
\hline $\mathrm{H}$ & -2.226636 & 0.914920 & -0.2 \\
\hline $\mathrm{H}$ & -4.415 & -1.134051 & -0.1 \\
\hline $\mathrm{H}$ & -1.508909 & -0.214030 & 3.2038 \\
\hline $\mathrm{H}$ & -0.612106 & -0.488499 & 1.756979 \\
\hline $\mathrm{H}$ & 5.154 & -2.987603 & \\
\hline $\mathrm{H}$ & 3.411832 & -3.231328 & 0.345519 \\
\hline $\mathrm{H}$ & 4.273962 & -2.750170 & -1.1188 \\
\hline $\mathrm{H}$ & 6.168096 & -0.718592 & \\
\hline $\mathrm{H}$ & 5.394886 & -0.395750 & -1.208427 \\
\hline $\mathrm{H}$ & 5.188131 & 0.728933 & 0.134181 \\
\hline $\mathrm{H}$ & -5.746981 & 0.736128 & -1.065395 \\
\hline $\mathrm{H}$ & -4.879475 & 1.282892 & 0.372948 \\
\hline $\mathrm{H}$ & -4.292028 & 1.718287 & -1.238335 \\
\hline $\mathrm{H}$ & -4.706570 & -0.896568 & -2.621643 \\
\hline $\mathrm{H}$ & -3.145659 & -0.080148 & -2.7167 \\
\hline $\mathrm{H}$ & -3.231364 & -1.769416 & -2.213815 \\
\hline
\end{tabular}




\section{Compound 2, conformation a (from ab initio conformational search)}

\begin{tabular}{|c|c|c|c|}
\hline $\mathrm{C}$ & 4.798779 & 2.188230 & 0.971713 \\
\hline $\mathrm{C}$ & 3.380788 & 2.014958 & 0.444221 \\
\hline O & 3.218437 & 0.535871 & 0.455016 \\
\hline C & 2.078077 & -0.041514 & .0907 \\
\hline $\mathrm{N}$ & 2.108325 & -1.374674 & 0.435 \\
\hline $\mathrm{N}$ & 1.138486 & -2.247505 & -0.01187 \\
\hline $\mathrm{C}$ & 1.366133 & -2.952027 & -1.2973 \\
\hline $\mathrm{C}$ & 1.273070 & -2.002747 & -2.49018 \\
\hline $\mathrm{C}$ & 2.694961 & -3.698699 & -1.2518 \\
\hline C & -0.131115 & -2.137655 & \\
\hline $\mathrm{N}$ & -0.256636 & -1.352513 & 1.624561 \\
\hline $\mathrm{N}$ & -1.498339 & -1.117161 & 2.175472 \\
\hline C & -1.992607 & -2.138164 & 3.09621 \\
\hline C & -2.412998 & -0.354598 & 1.45007 \\
\hline $\mathrm{N}$ & -1.899600 & 0.393524 & 0.4333 \\
\hline C & -2.766487 & 1.129932 & -0.4667 \\
\hline $\mathrm{C}$ & -2.417622 & 2.631058 & -0.5666 \\
\hline $\mathrm{C}$ & -2.487151 & 3.288730 & 0.8121 \\
\hline $\mathrm{C}$ & -1.067567 & 2.888137 & -1.23 \\
\hline $\mathrm{C}$ & -2.889859 & 0.483139 & -1.85 \\
\hline $\mathrm{N}$ & -2.408430 & -0.766249 & -1.99 \\
\hline O & -3.437583 & 1.090115 & -2.7 \\
\hline 0 & -3.604223 & -0.363776 & 1.75 \\
\hline 0 & -1.104097 & -2.680166 & -0.01 \\
\hline O & 1.111784 & 0.486730 & -0.4 \\
\hline $\mathrm{C}$ & 2.361975 & 2.636625 & \\
\hline C & 3.259514 & 2.540022 & -0. \\
\hline $\mathrm{H}$ & 5.514253 & 1.695335 & \\
\hline $\mathrm{H}$ & 5.044227 & 3.248365 & \\
\hline $\mathrm{H}$ & 4.890799 & 1.763673 & 1.97 \\
\hline $\mathrm{H}$ & 2.931866 & -1.765773 & 0.86 \\
\hline $\mathrm{H}$ & 0.555816 & -3.673572 & -1 \\
\hline $\mathrm{H}$ & 1.381125 & -2.568650 & -3.4 \\
\hline $\mathrm{H}$ & 0.314945 & -1.486878 & -2.50 \\
\hline $\mathrm{H}$ & 2.066019 & -1.255162 & -2.45 \\
\hline $\mathrm{H}$ & 2.804616 & -4.296891 & -2.15 \\
\hline $\mathrm{H}$ & 3.538296 & -3.007994 & -1.20 \\
\hline $\mathrm{H}$ & 2.743293 & -4.364332 & -0.39 \\
\hline $\mathrm{H}$ & 0.537948 & -0.940307 & 2.08 \\
\hline $\mathrm{H}$ & -2.878052 & -1.762459 & 3.59 \\
\hline $\mathrm{H}$ & -2.238267 & -3.061130 & 2.56 \\
\hline $\mathrm{H}$ & -1.217917 & -2.337314 & 3.833 \\
\hline $\mathrm{H}$ & -0.906069 & 0.385370 & 0.24 \\
\hline $\mathrm{H}$ & -3.767903 & 1.055564 & -0.04 \\
\hline $\mathrm{H}$ & -3.195784 & 3.062463 & -1.1971 \\
\hline $\mathrm{H}$ & -2.326407 & 4.364259 & 0.7289 \\
\hline $\mathrm{H}$ & -3.457898 & 3.125647 & 1.28 \\
\hline $\mathrm{H}$ & -1.720320 & 2.885998 & 1.47 \\
\hline $\mathrm{H}$ & -0.892711 & 3.960829 & -1.332 \\
\hline $\mathrm{H}$ & -0.245616 & 2.466445 & -0.663 \\
\hline $\mathrm{H}$ & -1.032124 & 2.455309 & -2.23 \\
\hline $\mathrm{H}$ & -2.546886 & -1.227098 & -2.882 \\
\hline $\mathrm{H}$ & -2.020258 & -1.304070 & -1.2355 \\
\hline $\mathrm{H}$ & 1.346537 & 2.499982 & $1.031 \mathrm{~S}$ \\
\hline $\mathrm{H}$ & 2.449286 & 2.192993 & 2.384 \\
\hline $\mathrm{H}$ & 2.557797 & 3.705372 & 1.476 \\
\hline $\mathrm{H}$ & 2.246922 & 2.435743 & -1.359 \\
\hline H & 3.532185 & 3.595291 & -0.99 \\
\hline & 3.941757 & 2.002284 & -1.64 \\
\hline
\end{tabular}




\section{Compound 2, conformation $\mathbf{b}$ (from ab initio conformational search)}

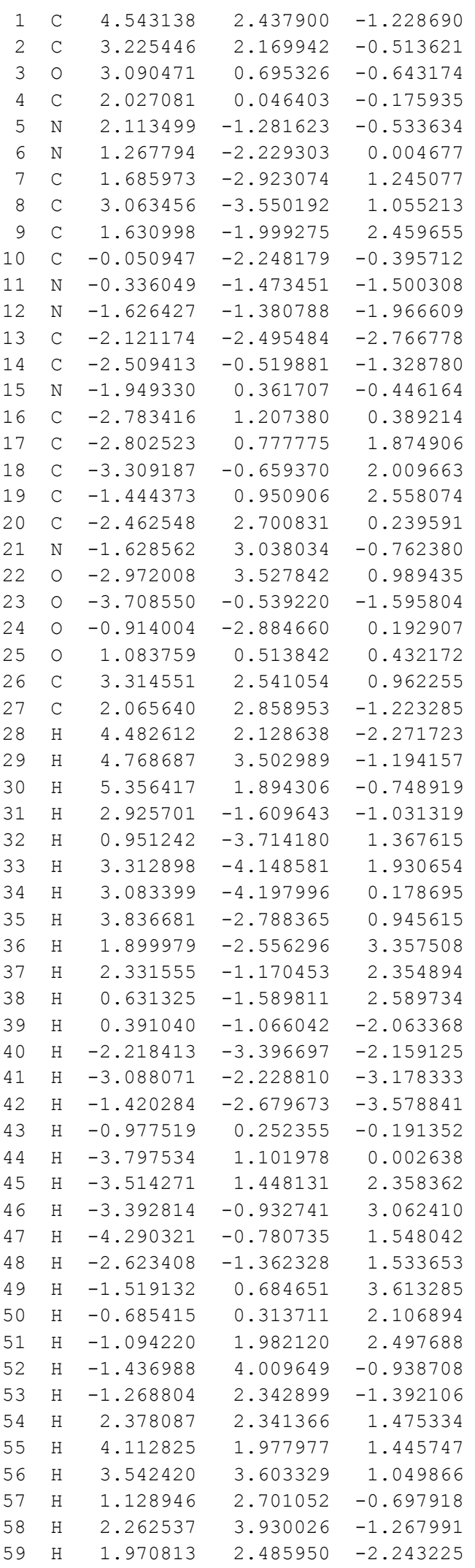




\section{Compound 2, conformation A-1 (from NMR parameters-restrained molecular dynamics)}

\begin{tabular}{|c|c|c|c|}
\hline & -3.219905 & -0.528572 & \\
\hline & -3.387348 & -2.007192 & 0.447535 \\
\hline & -4.806740 & -2.176420 & 0.972554 \\
\hline & -3.265566 & -2.530071 & -0.979376 \\
\hline & -2.372145 & -2.634100 & 1.396350 \\
\hline & -2.077262 & 0.045167 & 0.097889 \\
\hline & -1.111445 & -0.486460 & -0.415099 \\
\hline & -5.519489 & -1.679826 & 0.314899 \\
\hline & -5.056069 & -3.235753 & 1.018500 \\
\hline & -4.898916 & -1.753401 & 1.972328 \\
\hline & -2.252237 & -2.427976 & -1.355263 \\
\hline & -3.541169 & -3.584547 & -0.994494 \\
\hline & -3.945455 & -1.989335 & -1.637714 \\
\hline & -2.571756 & -3.702311 & 1.477829 \\
\hline & -1.355667 & -2.500450 & 1.037462 \\
\hline & -2.459587 & -2.192202 & 2.388888 \\
\hline & -2.104063 & 1.379117 & 41455 \\
\hline & -2.930061 & 1.7 & \\
\hline & -1.134588 & 2.249922 & \\
\hline & -1.363333 & 2.947400 & -1.3 \\
\hline & -0.552988 & 3.668578 & -1.360483 \\
\hline & -1.271197 & 1.992 & -2.48 \\
\hline & -0.313908 & 1.47 & -2.50 \\
\hline & -2.065124 & 1.2 & -2.4 \\
\hline & -1.378211 & 2.553598 & 17227 \\
\hline & -2.692082 & 3.694433 & -1.2 \\
\hline & -3.535650 & 3.00 & -1.212562 \\
\hline & -2.740220 & 4.36 & -0.399391 \\
\hline & -2.801985 & 4.2 & -2.164513 \\
\hline & 0.136772 & 2.1 & 0.501687 \\
\hline & 1.108403 & 2.680266 & -0.022355 \\
\hline & 0.2643 & & \\
\hline & -0.529475 & 45 & 864 \\
\hline & 1.507121 & 93 & 2081 \\
\hline & 2.00 & & 872 \\
\hline & 2.25 & 3. & .184 \\
\hline & 1.237571 & 2.3 & 6822 \\
\hline & 2.895904 & 1.7 & 3.5 \\
\hline & 2.418 & 0.3 & 1.4 \\
\hline & 3.60 & & 848 \\
\hline & 1.900 & -0.3 & 1973 \\
\hline & 0.907013 & -0.3 & 0.2 \\
\hline & 2.763813 & -1.1 & -0.4 \\
\hline & 3.76 & -1.0 & -0.0 \\
\hline & 2.413670 & -2.635883 & -0.562040 \\
\hline & 3.189298 & -3.069850 & -1.193919 \\
\hline & 2.487479 & -3.2888 & 0.818686 \\
\hline & 1.722751 & -2.88 & 1.483550 \\
\hline & 3.459572 & -3.1 & 6639 \\
\hline & 2.3 & -4 & 775 \\
\hline & & & \\
\hline & & & -2 . \\
\hline & 0.24 & -2.47 & -0.6 \\
\hline & 0.885303 & -3.9673 & -1.318226 \\
\hline & 2.882931 & -0.4927 & -1.862336 \\
\hline & 3.422537 & -1.1047 & -2.780409 \\
\hline & 2.406901 & 0.758925 & -2.003176 \\
\hline & 2.025891 & 1.300803 & -1.240079 \\
\hline & 2.544613 & 1.216973 & -2.888747 \\
\hline
\end{tabular}




\section{Compound 2, conformation A-2 (from NMR parameters-restrained molecular dynamics)}

\begin{tabular}{|c|c|c|c|}
\hline & -3.399170 & -0.023265 & 0.362070 \\
\hline & -3.864526 & -1.428682 & 0.519678 \\
\hline & -3.698990 & -2.177627 & -0.797895 \\
\hline & -3.107531 & -2.082636 & 1.669349 \\
\hline & -5.336094 & -1.244104 & 0.864593 \\
\hline & -2.133096 & 0.263223 & 0.080689 \\
\hline & -1.24447 & -0.516795 & -0.2 \\
\hline & -4.17506 & -1.625233 & $-1 \cdot 6$ \\
\hline & -2.65126 & -2.328909 & -1.03 \\
\hline & -4.18460 & -3.149975 & -0.715293 \\
\hline & -2.05455 & -2.204562 & \\
\hline & -3.202 & -1.482 & 2.5 \\
\hline & -3.534 & -3.066 & 1.8 \\
\hline & -5.80168 & -2.217 & 1.0 \\
\hline & -5.856814 & -0.729738 & \\
\hline & -5.44529 & -0.662050 & 1.7 \\
\hline & -1.927 & 1.619 & 0.2 \\
\hline & -2.706 & 2.213 & 0.4 \\
\hline & -0.795 & 2.232305 & -0.279 \\
\hline & -0.812 & 2.721917 & -1.681339 \\
\hline & 0.062 & 3.362748 & -1.751299 \\
\hline & -0.670 & 1.57 & -2.67 \\
\hline & -0.63 & 1.972345 & -3.6 \\
\hline & 0.24 & 1.010486 & -2.493695 \\
\hline & -1.51 & 0.89 & $-2 \cdot 6$ \\
\hline & -2.06 & 3.56 & -1.9 \\
\hline & -2.15 & 4.359 & $-1 \cdot 1$ \\
\hline & -1.998 & 4.012522 & -2.917813 \\
\hline & -2.965 & 2.951882 & $-1 \cdot 9$ \\
\hline & 0.40502 & 2.033264 & 0.3 \\
\hline & 1.47 & 2.372 & -0.1 \\
\hline & 0.338 & 1.406990 & 1.5 \\
\hline & -0.53491 & 1.104672 & 1.97 \\
\hline & 1.49 & 1.02 & 2.2 \\
\hline & 2.096 & 2.03 & 3.0 \\
\hline & 2.518 & 2.854 & 2.5 \\
\hline & 2.88120 & 1.574 & 3.6 \\
\hline & 1.32770 & 2.425708 & 3.761068 \\
\hline & 2.30811 & 0.062579 & 1.64 \\
\hline 0 & 3.46296 & -0.099843 & 2.02 \\
\hline N & 1.7553 & -0.677734 & 0.6 \\
\hline $\mathrm{H}$ & 0.79091 & -0.547413 & 0.371448 \\
\hline & 2.585722 & -1.612887 & -0.097145 \\
\hline 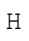 & 3.219350 & -2.135649 & 0.618533 \\
\hline C & 1.761593 & -2.678709 & -0.856612 \\
\hline $\mathrm{H}$ & 2.510839 & -3.375882 & -1.235145 \\
\hline 0 & 0.83560 & -3.440864 & 0.093900 \\
\hline & 0.377855 & -4.284274 & -0.424544 \\
\hline & 0.031129 & -2.802947 & 0.458489 \\
\hline $\mathrm{H}$ & 1.382298 & -3.827482 & 0.955557 \\
\hline $\mathrm{C}$ & 0.995713 & -2.118947 & -2.060352 \\
\hline $\mathrm{H}$ & 0.248854 & -1.387689 & -1.759486 \\
\hline H & 1.667116 & -1.648518 & -2.778928 \\
\hline $\mathrm{H}$ & 0.474909 & -2.928455 & -2.573863 \\
\hline 0 & 3.570309 & -0.938926 & -1.066657 \\
\hline 0 & 4.481123 & -1.603102 & -1.556823 \\
\hline & 3.358802 & 0.353703 & -1.368835 \\
\hline & 2.658063 & 0.923657 & -0.913621 \\
\hline & 4.007834 & 0.804576 & -1.99194 \\
\hline
\end{tabular}




\section{Compound 2, conformation B (from NMR parameters-restrained molecular dynamics)}

\begin{tabular}{|c|c|c|c|}
\hline O & 2.420343 & 1.747985 & -0.853621 \\
\hline C & 1.823499 & 3.110746 & -0.791818 \\
\hline C & 1.847255 & 3.613064 & 0.647107 \\
\hline C & 2.772516 & 3.915717 & -1.669879 \\
\hline C & 0.418240 & 3.081650 & -1.382874 \\
\hline C & 1.844274 & 0.711761 & -0.253500 \\
\hline O & 0.880637 & 0.732284 & 0.489825 \\
\hline $\mathrm{H}$ & 1.534875 & 4.657268 & 0.663985 \\
\hline $\mathrm{H}$ & 2.857122 & 3.550940 & 1.052744 \\
\hline $\mathrm{H}$ & 1.175218 & 3.037283 & 6574 \\
\hline $\mathrm{H}$ & 3.786586 & 3.877255 & -1.273129 \\
\hline $\mathrm{H}$ & 2.779319 & 3.524035 & -2.686621 \\
\hline $\mathrm{H}$ & 2.450383 & 4.955819 & -1.699968 \\
\hline $\mathrm{H}$ & 0.063659 & 4.105715 & -1.500520 \\
\hline $\mathrm{H}$ & 0.43 & 2.612756 & $-2 \cdot 3$ \\
\hline $\mathrm{H}$ & -0.282 & 2.550924 & -0.74 \\
\hline $\mathrm{N}$ & 2.474 & -0.450802 & -0.63 \\
\hline $\mathrm{H}$ & 3.266655 & -0.411187 & $-1 \cdot 25$ \\
\hline $\mathrm{N}$ & 2.189080 & -1.655469 & -0.026542 \\
\hline C & 2.998 & -2.053720 & 1.1 \\
\hline $\mathrm{H}$ & 2.675 & -3.068680 & 1.36 \\
\hline C & 4.47 & -2.070510 & 0.7 \\
\hline $\mathrm{H}$ & 4.84 & -1.065712 & 0.563643 \\
\hline $\mathrm{H}$ & 5.058 & -2.462309 & 1.612583 \\
\hline $\mathrm{H}$ & 4.658348 & -2.701250 & -0.093082 \\
\hline C & 2.715 & -1.173021 & 2.362724 \\
\hline $\mathrm{H}$ & 1.657 & -1.187456 & 2.61 \\
\hline $\mathrm{H}$ & 3.285873 & -1.53 & 3.2 \\
\hline $\mathrm{H}$ & 3.009 & -0.139955 & 2.175043 \\
\hline C & 0.964310 & -2.247742 & -0.265248 \\
\hline $\mathrm{O}$ & 0.513 & -3.143464 & 0.433261 \\
\hline $\mathrm{N}$ & 0.27 & -1.748408 & -1.353133 \\
\hline $\mathrm{H}$ & 0.70 & -1.09 & -1.99 \\
\hline $\mathrm{N}$ & -1.00 & -2.158721 & -1.621183 \\
\hline C & -1.16 & -3.420638 & -2.331445 \\
\hline $\mathrm{H}$ & -0.599 & -3.380744 & -3.260449 \\
\hline $\mathrm{H}$ & -0.808 & -4.253334 & -1.723691 \\
\hline $\mathrm{H}$ & -2.218 & -3.559505 & -2.555620 \\
\hline C & -2.05 & -1.585925 & -0.91 \\
\hline O & -3.210 & -2.002275 & -1.0532 \\
\hline $\mathrm{N}$ & -1.734944 & -0.543271 & -0.114112 \\
\hline $\mathrm{H}$ & -0.784437 & -0.204648 & -0.050790 \\
\hline C & -2.743812 & 0.159858 & 0.668676 \\
\hline $\mathrm{H}$ & -3.542733 & -0.551022 & 0.882726 \\
\hline C & -2.166 & 0.675554 & 1.992310 \\
\hline $\mathrm{H}$ & -1.399220 & 1.413167 & 1.751129 \\
\hline $\mathrm{C}$ & -3.260186 & 1.357397 & 2.819803 \\
\hline $\mathrm{H}$ & -3.698764 & 2.201286 & 2.290335 \\
\hline $\mathrm{H}$ & -4.057387 & 0.650164 & 3.062635 \\
\hline $\mathrm{H}$ & -2.848012 & 1.726706 & 3.759571 \\
\hline C & -1.523460 & -0.462824 & 2.789246 \\
\hline $\mathrm{H}$ & -0.715620 & -0.941211 & 2.239827 \\
\hline $\mathrm{H}$ & -1.113987 & -0.080592 & 3.725156 \\
\hline $\mathrm{H}$ & -2.263921 & -1.228063 & 3.035751 \\
\hline C & -3.311330 & 1.281759 & -0.225310 \\
\hline O & -2.929275 & 2.444499 & -0.160616 \\
\hline $\mathrm{N}$ & -4.235935 & 0.852240 & -1.112365 \\
\hline $\mathrm{H}$ & -4.361570 & -0.140572 & -1.251621 \\
\hline $\mathrm{H}$ & -4.530 & 1.481903 & -1.8412 \\
\hline
\end{tabular}




\section{Compound $\mathbf{3}$, conformation a}

\begin{tabular}{|c|c|c|c|}
\hline C & .642845 & -2.676130 & \\
\hline 2 & .122790 & -3.010713 & -1.660302 \\
\hline & 168813 & -2.701353 & \\
\hline & 87262 & & \\
\hline 5 & .368895 & -1.423639 & \\
\hline . & 108811 & -0.308580 & \\
\hline & & & \\
\hline 8 & .287678 & -1.449554 & 887756 \\
\hline & .517492 & 0.074163 & -0.98 \\
\hline & .452661 & & 902 \\
\hline & .133839 & 0.611684 & $590^{\circ}$ \\
\hline & .332447 & & \\
\hline C & 0.383144 & .116531 & 9471 \\
\hline C & -0.228713 & .458055 & 7060 \\
\hline $\mathrm{N}$ & 0.019222 & .073507 & \\
\hline C & -0.808286 & .604172 & 3836 \\
\hline C & 0.300093 & .094031 & -2 . \\
\hline & -1.196858 & .567069 & \\
\hline & 1.147898 & .536738 & \\
\hline 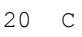 & -2.280329 & 247577 & \\
\hline . & -2.400089 & 05213 & \\
\hline 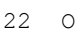 & -3.211968 & 279 & \\
\hline 0 & -0.926442 & & \\
\hline & 2.992686 & & \\
\hline 0 & 1.314358 & 5999 & 492 \\
\hline C & -1.155100 & -2.262 & \\
\hline & 344 & & \\
\hline$c$ & -3.710491 & & -0 . \\
\hline 9 & -3.499920 & -0.68 & \\
\hline & -2.5 & & \\
\hline C & -2.338683 & -1.81 & 493 \\
\hline 2 & -3.042093 & -2.98 & \\
\hline & -3.9 & & \\
\hline $\mathrm{C}$ & -4.202094 & -1.8 & \\
\hline $\mathrm{H}$ & 1.600720 & -3.1 & -3.2 \\
\hline & -0.0 & & \\
\hline $\mathrm{H}$ & 0.763729 & -1 & -3 . \\
\hline $\mathrm{H}$ & 2.558612 & -2 & 789 \\
\hline & & & \\
\hline $\mathrm{H}$ & .329402 & $-1 \cdot 3$ & 44 \\
\hline $\mathrm{H}$ & 5.249149 & -1 & 614 \\
\hline & .911287 & -2 & \\
\hline $\mathrm{H}$ & .547236 & & -1 \\
\hline 4 & 4.089473 & -0.7 & -1 \\
\hline & & & -1 \\
\hline $\mathrm{H}$ & 0.717498 & -0.3 & \\
\hline 7 & -0.346599 & 2.9 & 43 \\
\hline & & & \\
\hline $\mathrm{H}$ & .37853 & & \\
\hline $\mathrm{H}$ & .451673 & 7 & -0 . \\
\hline $\mathrm{H}$ & -0.766287 & & -1 \\
\hline $\mathrm{H}$ & -0.323517 & 6 & -2 \\
\hline $\mathrm{H}$ & -0.8 & 5 & -4 . \\
\hline $\mathrm{H}$ & -2.218569 & & \\
\hline $\mathrm{H}$ & -1.234145 & 0 & \\
\hline & 1.508903 & 7 & -3 . \\
\hline $\mathrm{H}$ & 1.215233 & & -2 . \\
\hline $\mathrm{H}$ & 1.811811 & & \\
\hline $\mathrm{H}$ & -1.448845 & 80 & -0.2 \\
\hline $\mathrm{H}$ & -1.041512 & & -1 \\
\hline $\mathrm{H}$ & -1.957869 & -2.5 & -1 \\
\hline H & -0.385727 & -4.74 & -0.4 \\
\hline $\mathrm{H}$ & -0.811114 & -4.8 & -2.1 \\
\hline $\mathrm{H}$ & & -5 & -1 \\
\hline $\mathrm{H}$ & -4.186240 & 1.3 & \\
\hline $\mathrm{H}$ & -4.316475 & 0.3 & -0.92 \\
\hline $\mathrm{H}$ & -2.017374 & & \\
\hline $\mathrm{H}$ & -1.608858 & -1.7966 & 3.37 \\
\hline $\mathrm{H}$ & -2.857848 & -3.8800 & 2.88256 \\
\hline $\mathrm{H}$ & & & \\
\hline & -4.913885 & -1.877825 & -0.29704 \\
\hline
\end{tabular}




\section{Compound $\mathbf{3}$, conformation $\mathbf{b}$}

\begin{tabular}{|c|c|c|c|c|}
\hline 1 & C & 4.564980 & 0.810850 & -0.176187 \\
\hline 2 & $\mathrm{C}$ & 4.557349 & -0.558613 & -0.844663 \\
\hline & 0 & 3.626638 & -1.463604 & -0.117907 \\
\hline & $\mathrm{C}$ & 2.334245 & -1.181059 & 0.005604 \\
\hline 5 & $\mathrm{~N}$ & 1.741288 & -2.100089 & 0.847071 \\
\hline & $\mathrm{N}$ & 0.367846 & -2.211106 & 0.903674 \\
\hline 1 & C & -0.303862 & -3.165124 & -0.006419 \\
\hline 8 & C & 0.117444 & -2.938416 & -1.455485 \\
\hline 9 & $\mathrm{C}$ & -0.056612 & -4.600921 & 0.451032 \\
\hline 1 & $\mathrm{C}$ & 350009 & -1.317 & 65242 \\
\hline 1 & $\mathrm{~N}$ & 0.407654 & -0.454156 & 2.430070 \\
\hline 12 & $\mathrm{~N}$ & -0.188674 & 0.631825 & 3.032346 \\
\hline 13 & $\mathrm{C}$ & -0.849107 & 0.387262 & 4.309357 \\
\hline 14 & $\mathrm{C}$ & -0.567653 & 1.714307 & 2.242254 \\
\hline & $\mathrm{N}$ & -0.049043 & 1.737682 & 0.984544 \\
\hline 6 & $\mathrm{C}$ & -0.334966 & 2.818250 & 0.062797 \\
\hline 17 & C & 0.916617 & 3.180111 & -0.766551 \\
\hline 18 & C & 4648 & 4.199 & 58727 \\
\hline 19 & C & 2.019685 & 3.706 & 53575 \\
\hline 20 & C & -1.52 & 2.46 & 363 \\
\hline 21 & 0 & -1.307127 & 1.322313 & -1.496543 \\
\hline 22 & 0 & -2.512689 & 3.150154 & -0.943849 \\
\hline 23 & 0 & -1.286779 & 2.603689 & 2.693870 \\
\hline 24 & 0 & -1.572172 & -1.271056 & 1.661574 \\
\hline 25 & 0 & 1.731787 & -0.244805 & -0.484439 \\
\hline 6 & C & 4.156566 & -0.494278 & -2.313989 \\
\hline 7 & C & 5.896105 & -1.262894 & 70385 \\
\hline 28 & C & -2.331894 & 0.854 & 25088 \\
\hline 29 & C & -3.2 & -0.15 & -1 \\
\hline 30 & C & -3.105792 & -1.508064 & -2.059967 \\
\hline 31 & $\mathrm{C}$ & -3.922223 & -2.446399 & -1.437032 \\
\hline 32 & $\mathrm{C}$ & -4.877369 & -2.032674 & -0.515452 \\
\hline 33 & $\mathrm{C}$ & -5.016309 & -0.677884 & -0.223747 \\
\hline 34 & $\mathrm{C}$ & -4.201060 & 0.257546 & -0.847867 \\
\hline 35 & $\mathrm{H}$ & 4.767746 & 0.710732 & 0.890156 \\
\hline 36 & $\mathrm{H}$ & 5.354036 & 1.418432 & -0.618917 \\
\hline 37 & $\mathrm{H}$ & 3.6 & 1.322 & 10206 \\
\hline 38 & $\mathrm{H}$ & 2.28 & -2.876 & 908 \\
\hline 39 & $\mathrm{H}$ & -1.360248 & -2.932690 & 0.093653 \\
\hline 40 & $\mathrm{H}$ & -0.463863 & -3.591198 & -2.105672 \\
\hline 41 & $\mathrm{H}$ & -0.050651 & -1.906152 & -1.753565 \\
\hline 42 & $\mathrm{H}$ & 1.171734 & -3.176176 & -1.604676 \\
\hline 43 & $\mathrm{H}$ & -0.602649 & -5.294238 & -0.188815 \\
\hline 44 & $\mathrm{H}$ & 1.002355 & -4.859584 & 0.389173 \\
\hline 45 & $\mathrm{H}$ & -0.389601 & -4.743875 & 1.478755 \\
\hline 46 & $\mathrm{H}$ & 1.411027 & -0.508 & 2.457385 \\
\hline 47 & $\mathrm{H}$ & -1.7 & & \\
\hline 4 & $\mathrm{H}$ & -0.145 & -0.110 & 4.973243 \\
\hline 49 & $\mathrm{H}$ & -1.140978 & 1.338623 & 4.740285 \\
\hline 50 & $\mathrm{H}$ & 0.496786 & 0.965235 & 0.628431 \\
\hline 51 & $\mathrm{H}$ & -0.650751 & 3.682263 & 0.642361 \\
\hline 52 & $\mathrm{H}$ & 1.263790 & 2.259721 & -1.241843 \\
\hline 53 & $\mathrm{H}$ & 1.486609 & 4.464618 & -2.410209 \\
\hline 54 & $\mathrm{H}$ & -0.138729 & 3.812122 & -2.577347 \\
\hline 55 & $\mathrm{H}$ & 0.173865 & 5.113747 & -1.425865 \\
\hline 56 & $\mathrm{H}$ & 2.928996 & 3.899707 & -0.416065 \\
\hline 57 & $\mathrm{H}$ & 1.7 & 2007 & 25362 \\
\hline 58 & $\mathrm{H}$ & 2.260298 & 2.993519 & 0.941148 \\
\hline 59 & $\mathrm{H}$ & 4.089263 & -1.498954 & -2.731264 \\
\hline 60 & $\mathrm{H}$ & 3.201465 & 0.007271 & -2.440354 \\
\hline 61 & $\mathrm{H}$ & 4.917660 & 0.056265 & -2.866807 \\
\hline 62 & $\mathrm{H}$ & 5.862278 & -2.262329 & -1.102920 \\
\hline 63 & $\mathrm{H}$ & 6.677213 & -0.693030 & -1.172079 \\
\hline 64 & $\mathrm{H}$ & 6.150230 & -1.346549 & 0.385798 \\
\hline 65 & $\mathrm{H}$ & -2.885269 & 1.716886 & -2.789351 \\
\hline 66 & $\mathrm{H}$ & -1.772577 & 0.408426 & -3.242572 \\
\hline 67 & $\mathrm{H}$ & 1591 & -1.833336 & -2.774168 \\
\hline 68 & $\mathrm{H}$ & -3.808571 & -3.496838 & -1.668828 \\
\hline 69 & $\mathrm{H}$ & -5.511607 & -2.760329 & -0.027261 \\
\hline 70 & $\mathrm{H}$ & -5.760614 & -0.351577 & 0.490216 \\
\hline & $\mathrm{H}$ & -4.303667 & 1.309472 & -0.619573 \\
\hline
\end{tabular}




\section{Compound 3 , conformation $\mathrm{C}$}

\begin{tabular}{|c|c|c|c|}
\hline C & .987946 & -0.270168 & \\
\hline 2 & .878062 & 0.772661 & \\
\hline & 629261 & 33844 & \\
\hline & 9705 & 32173 & 0 \\
\hline 5 & 838321 & -1.158527 & 0.53250 \\
\hline & 177510 & 21502 & \\
\hline & 477224 & 7107 & \\
\hline 8 & 977434 & -3.823207 & .99904 \\
\hline & 854840 & 91439 & \\
\hline 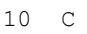 & 0.074113 & 3149 & \\
\hline $\mathrm{N}$ & -0.262804 & -0.978041 & -1.05338 \\
\hline N & 1.165605 & -0.840164 & \\
\hline C & 0.699791 & -1.184843 & -342578 \\
\hline C & -2.514145 & -0.713585 & -1.79997 \\
\hline $\mathrm{N}$ & 2.819735 & 679970 & \\
\hline & 75531 & 3173 & \\
\hline C & 69899 & 78511 & 16125 \\
\hline & -5.029051 & & \\
\hline & -3.733856 & 9343 & \\
\hline 0 & -3.973106 & 9028 & 41320 \\
\hline 0 & -2.741 & 4408 & \\
\hline & -4.9 & 9101 & \\
\hline 3 & -3.356020 & 39365 & 69170 \\
\hline 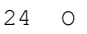 & -0.585 & 4196 & -0.93134 \\
\hline & 3.378421 & 6897 & \\
\hline 6 & 5.166990 & 2347 & \\
\hline C & 4.619 & & $-1.252 \varepsilon$ \\
\hline & -2.486 & 658 & \\
\hline 9 & -1.023 & 6407 & \\
\hline$C$ & -0.599 & & -0 . \\
\hline & 0.757 & 650 & \\
\hline 2 & 1.70 & 3692 & 0.08523 \\
\hline C & 99 & & \\
\hline $\mathrm{C}$ & -0.069317 & 3178 & \\
\hline $\mathrm{H}$ & 5.785966 & -1 & 749001 \\
\hline $\mathrm{H}$ & 82 & & \\
\hline $\mathrm{H}$ & 907 & -0 . & \\
\hline 8 & 309 & -0 . & 0 \\
\hline & 52 & -4 & \\
\hline $\mathrm{H}$ & 06 & -4 . & 896 \\
\hline $\mathrm{H}$ & 3.398560 & -3 & -0.00368 \\
\hline & 53 & & \\
\hline $\mathrm{H}$ & 9 & -4 & \\
\hline H & 1901 & -2 . & 4697 \\
\hline $\mathrm{H}$ & -0.21 & -3 & \\
\hline $\mathrm{H}$ & 30 & -0 & 832 \\
\hline $\mathrm{H}$ & -1.49 & -0 . & 3406 \\
\hline & & & -3 \\
\hline $\mathrm{H}$ & -0.43 & -2.2 & -3 . \\
\hline $\mathrm{H}$ & -2.0 & -0 . & 15359 \\
\hline & -4.7 & & -0 . \\
\hline $\mathrm{H}$ & -5.585496 & -0.4 & 1.456 \\
\hline $\mathrm{H}$ & -5.4 & -2 . & 4005 \\
\hline $\mathrm{H}$ & -5.7 & -2 . & 15590 \\
\hline $\mathrm{H}$ & -4.137 & -2 & \\
\hline $\mathrm{H}$ & -4.214229 & -1 & \\
\hline $\mathrm{H}$ & -2.81 & -1 & 888 \\
\hline $\mathrm{H}$ & -3.4 & -0. & \\
\hline & & & 172070 \\
\hline $\mathrm{H}$ & & & 4209 \\
\hline $\mathrm{H}$ & 6.08 & 2.334603 & \\
\hline I & & & -1.18144 \\
\hline $\mathrm{H}$ & & & -1.53058 \\
\hline $\mathrm{H}$ & & & -2.03238 \\
\hline $\mathrm{H}$ & -3.109755 & & \\
\hline $\mathrm{H}$ & -2.772587 & 3.278162 & 1.69897 \\
\hline $\mathrm{H}$ & -1.330757 & 5.3 & -0.44094 \\
\hline $\mathrm{H}$ & 1.074352 & 5.839838 & -0.73955 \\
\hline $\mathrm{H}$ & 2.755277 & 4.157866 & -0.05536 \\
\hline $\mathrm{H}$ & & & \\
\hline & -0389675 & 1.566645 & 1.26462 \\
\hline
\end{tabular}




\section{Compound 4, conformation a}

\begin{tabular}{|c|c|c|c|}
\hline $\mathrm{C}$ & 6.273152 & -0.490975 & -0.776041 \\
\hline C & 5.549755 & 0.492662 & 0.136741 \\
\hline O & 4.187617 & 0.754881 & -0.395471 \\
\hline C & 3.273979 & -0.208229 & -0.489081 \\
\hline $\mathrm{N}$ & 2.080225 & 0.350803 & -0.929100 \\
\hline $\mathrm{N}$ & 1.067610 & -0.486562 & -1.347222 \\
\hline C & 0.126413 & -0.953981 & -0.460810 \\
\hline $\mathrm{N}$ & 0.028933 & -0.255069 & 0.716990 \\
\hline $\mathrm{N}$ & -0.785606 & -0.711443 & 1.735252 \\
\hline C & -2.175021 & -0.651000 & 1.599019 \\
\hline $\mathrm{N}$ & -2.635768 & 0.223680 & 0.673905 \\
\hline C & -4.041062 & 0.319473 & 0.335645 \\
\hline C & -4.401094 & 1.722421 & -0.201163 \\
\hline C & -4.191722 & 2.791337 & 0.872401 \\
\hline C & -3.655253 & 2.071172 & -1.493747 \\
\hline C & -4.528917 & -0.764831 & -0.642107 \\
\hline O & -5.729255 & -0.848195 & -0.891406 \\
\hline 0 & -2.888424 & -1.343077 & 2.314699 \\
\hline O & -0.621450 & -1.879868 & -0.751469 \\
\hline O & 3.392901 & -1.382966 & -0.220691 \\
\hline C & 5.449322 & -0.002446 & 1.575092 \\
\hline C & 6.193961 & 1.871490 & 0.076117 \\
\hline $\mathrm{N}$ & -3.605255 & -1.570630 & -1.191338 \\
\hline $\mathrm{H}$ & 6.259915 & -0.130628 & -1.804683 \\
\hline $\mathrm{H}$ & 7.311722 & -0.576097 & -0.456348 \\
\hline $\mathrm{H}$ & 5.814554 & -1.475017 & -0.738722 \\
\hline $\mathrm{H}$ & 2.112788 & 1.256952 & -1.370650 \\
\hline $\mathrm{H}$ & 1.264606 & -1.108963 & -2.119116 \\
\hline $\mathrm{H}$ & 0.716554 & 0.439636 & 0.957787 \\
\hline $\mathrm{H}$ & -0.481462 & -1.567852 & 2.178771 \\
\hline $\mathrm{H}$ & -1.964448 & 0.736738 & 0.129146 \\
\hline $\mathrm{H}$ & -4.611117 & 0.146756 & 1.247913 \\
\hline $\mathrm{H}$ & -5.465245 & 1.667111 & -0.432321 \\
\hline $\mathrm{H}$ & -4.518666 & 3.765124 & 0.506320 \\
\hline $\mathrm{H}$ & -4.758570 & 2.560126 & 1.775460 \\
\hline $\mathrm{H}$ & -3.139231 & 2.875130 & 1.148148 \\
\hline $\mathrm{H}$ & -4.023217 & 3.016721 & -1.892107 \\
\hline $\mathrm{H}$ & -2.582181 & 2.194079 & -1.328287 \\
\hline $\mathrm{H}$ & -3.792240 & 1.307931 & -2.260446 \\
\hline $\mathrm{H}$ & 4.866646 & 0.696496 & 2.175213 \\
\hline $\mathrm{H}$ & 4.986565 & -0.984060 & 1.624080 \\
\hline $\mathrm{H}$ & 6.450848 & -0.066649 & 2.000209 \\
\hline $\mathrm{H}$ & 5.640157 & 2.580235 & 0.691011 \\
\hline $\mathrm{H}$ & 7.216791 & 1.814548 & 0.446477 \\
\hline $\mathrm{H}$ & 6.215278 & 2.239384 & -0.949223 \\
\hline $\mathrm{H}$ & -3.921519 & -2.292872 & -1.817084 \\
\hline $\mathrm{H}$ & -2.621704 & -1.539023 & -0.958634 \\
\hline
\end{tabular}




\section{Compound 4, conformation b}

\begin{tabular}{|c|c|c|c|}
\hline C & 5.370854 & -1.085657 & 0.009176 \\
\hline C & 3.854721 & -1.106579 & 0.152378 \\
\hline O & 3.476884 & 0.260875 & -0.290837 \\
\hline C & 2.207983 & 0.654934 & -0.324031 \\
\hline $\mathrm{N}$ & 2.125596 & 1.940045 & -0.817232 \\
\hline $\mathrm{N}$ & .914714 & 2.595290 & -0.69 \\
\hline C & 0.379268 & 2.673179 & 0.58 \\
\hline $\mathrm{N}$ & -0.988708 & 2.694370 & 0.636106 \\
\hline $\mathrm{N}$ & -1.793429 & 2.548984 & -0.476998 \\
\hline C & -2.51 & 64 & -0.701 \\
\hline $\mathrm{N}$ & -1.97 & 0.226 & -0.204 \\
\hline C & -2.64 & -1.04 & -0.4 \\
\hline C & -1.66 & -2.23 & -0.55 \\
\hline C & -0.76 & -2.06 & -1.780448 \\
\hline C & -0.860382 & -2.49 & 0.721188 \\
\hline C & -3.713706 & -1.37 & 0.63 \\
\hline O & -4.50 & -2.292 & 0.4 \\
\hline O & -3.559 & 1.41 & $-1 \cdot 3$ \\
\hline O & 1.058322 & 2.7 & 1.5 \\
\hline O & 1.227 & 0.006825 & -0.009115 \\
\hline C & 3.229732 & -2.140489 & -0.777862 \\
\hline C & 3.44 & $-1 \cdot 30$ & 1.607540 \\
\hline $\mathrm{N}$ & -3.71 & -0.61 & 1.7 \\
\hline $\mathrm{H}$ & 5.80 & -0.30 & 0.639715 \\
\hline $\mathrm{H}$ & 5.780 & -2.04 & 0830 \\
\hline $\mathrm{H}$ & 5.654891 & -0.895149 & -1.025346 \\
\hline $\mathrm{H}$ & 2.950 & 2.51 & -0.792071 \\
\hline $\mathrm{H}$ & 0.260 & 2.38 & $-1 \cdot 4$ \\
\hline $\mathrm{H}$ & -1.414513 & 2.81 & 3661 \\
\hline $\mathrm{H}$ & -2.31 & 3.3 & -0.7 \\
\hline $\mathrm{H}$ & -1.015 & & \\
\hline $\mathrm{H}$ & -3.203509 & -0.971916 & $-1 \cdot 3$ \\
\hline $\mathrm{H}$ & -2.312831 & -3.09 & -0.725495 \\
\hline $\mathrm{H}$ & -0.143363 & -2.95 & -1.91 \\
\hline $\mathrm{H}$ & -1.3551 & -1.92 & -2.68 \\
\hline $\mathrm{H}$ & -0.0973 & -1.21 & -1.669128 \\
\hline $\mathrm{H}$ & -0.250 & -3.39 & 0.594241 \\
\hline $\mathrm{H}$ & -0.1822 & -1.67 & 9521 \\
\hline $\mathrm{H}$ & -1.512181 & -2.657341 & 1.580672 \\
\hline $\mathrm{H}$ & 2.1495 & -2.167448 & -0.672515 \\
\hline $\mathrm{H}$ & 3.480739 & -1.916743 & -1.814781 \\
\hline $\mathrm{H}$ & 3.6296 & -3.124634 & -0.533652 \\
\hline $\mathrm{H}$ & 2.363139 & -1.325928 & 1.713256 \\
\hline $\mathrm{H}$ & 3.850347 & -2.248674 & 1.966897 \\
\hline $\mathrm{H}$ & 3.845758 & -0.500185 & 2.225235 \\
\hline $\mathrm{H}$ & -4.387556 & -0.805032 & 2.471342 \\
\hline $\mathrm{H}$ & -3.050282 & 0.124447 & 1.874497 \\
\hline
\end{tabular}




\section{Compound 4, conformation c}

\begin{tabular}{|c|c|c|c|}
\hline & -2.882225 & -2.026128 & 1.098646 \\
\hline , & -3.312278 & -1.599956 & -0.300245 \\
\hline & -2.904372 & -0.190434 & -0.523658 \\
\hline & -3.431094 & 0.805707 & 0.180855 \\
\hline & -2.943314 & 2.036066 & -0.233662 \\
\hline & -1.748943 & 2.159460 & -0.902903 \\
\hline & -0.57 & 2.18 & -0.2 \\
\hline & & 2.31 & -1.0 \\
\hline & 1.7 & 2.701 & -0.3 \\
\hline & 2.578336 & 1.752360 & \\
\hline & 2.403093 & 0.475849 & -0.2 \\
\hline & 3.07 & -0.64 & \\
\hline & 2.08 & -1.5 & 1.1 \\
\hline & 1.38 & -0.86 & 2.2 \\
\hline & 1.07 & -2.21 & 0.1 \\
\hline & 3.98 & -1.43 & -0.5 \\
\hline$U$ & 4.5 & -2.45 & -0.1 \\
\hline 0 & 3.4 & 2.1 & 0.9 \\
\hline & -0.5 & 2.0 & 1. \\
\hline 0 & -4.27 & 0.733 & 1.0 \\
\hline C & -2.52 & -2.342 & $-1 \cdot 3$ \\
\hline $\mathrm{C}$ & -4.81 & -1.76 & -0.5 \\
\hline & 4.14 & -0.91 & -1.8 \\
\hline & -3.4 & $-1 \cdot 4$ & 1.8 \\
\hline & -3.06 & -3.0 & 1.2 \\
\hline $\mathrm{H}$ & -1.81 & $-1 \cdot 8$ & 1.2 \\
\hline H & -3.16 & 2.7 & 0.3 \\
\hline $\mathrm{H}$ & -1.79 & 2.31 & -1.896266 \\
\hline & 0.45 & 2.6 & -1.9 \\
\hline 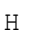 & 1.70 & 3.5 & 0.1 \\
\hline $\mathrm{H}$ & 1.58 & 0.29 & -0.8 \\
\hline $\mathrm{H}$ & 3.74 & -0.22 & \\
\hline $\mathrm{H}$ & 2.70 & -2.39 & 1.5 \\
\hline 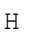 & 0.72 & -1.5 & 2.7 \\
\hline $\mathrm{H}$ & 2.101 & -0.45 & 2.9 \\
\hline $\mathrm{H}$ & 0.769 & -0.03 & 1.8 \\
\hline $\mathrm{H}$ & 0.447 & -2.93 & \\
\hline $\mathrm{H}$ & 0.403559 & -1.45 & -0.2 \\
\hline $\mathrm{H}$ & 1.559253 & -2.72 & -0.6 \\
\hline $\mathrm{H}$ & -2.818791 & -2.000518 & -2.365550 \\
\hline $\mathrm{H}$ & -1.457547 & -2.183190 & -1.250566 \\
\hline $\mathrm{H}$ & -2.727119 & -3.411 & -1.300914 \\
\hline $\mathrm{H}$ & -5.088484 & -1.35 & -1.504144 \\
\hline $\mathrm{H}$ & -5.057063 & -2.82 & -0.52300 \\
\hline $\mathrm{H}$ & -5.390385 & -1.260764 & 0.23930 \\
\hline $\mathrm{H}$ & 4.77 & -1.36 & -2.44 \\
\hline & 3.71 & -0.047 & -2. \\
\hline
\end{tabular}




\section{Compound 4, conformation d}

\begin{tabular}{|c|c|c|c|}
\hline & -2.163026 & 2.301254 & 0.582829 \\
\hline$C$ & -2.906088 & 1.761227 & -0.633959 \\
\hline & -3.088372 & 0.297946 & -0.483229 \\
\hline & -3.759243 & -0.226088 & 0.537389 \\
\hline & -3.693328 & -1.610767 & 0.517621 \\
\hline & -2.689565 & -2.284527 & -0.146838 \\
\hline & -1.378541 & -2.085276 & \\
\hline & -0.457091 & -2.678286 & -0.598 \\
\hline & 88061 & -2.399 & -0.420 \\
\hline & 1.31 & -1.09 & -0.6 \\
\hline & 2.54 & -0.82 & -0.1 \\
\hline & 3.11 & 0.51 & -0.1 \\
\hline & 3.12 & 1.16 & \\
\hline & & 588 & \\
\hline & 3.96 & 240 & 2.2 \\
\hline & 4.5 & 641 & -0.7 \\
\hline & 5.16 & 1.60 & -0.72 \\
\hline & 0.66 & -0.296 & -1.298 \\
\hline & -1.068 & -1.51 & 1.273 \\
\hline & -4.41 & 0.36 & 1.3 \\
\hline & -4.25 & 2.43 & -0.8 \\
\hline & -2.04 & 1.85 & -1.8 \\
\hline & 4.90 & -0.53 & $-1 \cdot 4$ \\
\hline & -1.24 & 1.73 & \\
\hline & -1.90 & 3.34 & 0.409 \\
\hline & -2.77 & 2.2 & 1.4 \\
\hline & -4.01 & -2.04 & 1.36 \\
\hline & -2.90 & -2.52 & -1.10 \\
\hline & -0.716 & -2.923827 & -1.54 \\
\hline & 1.305527 & -2.926338 & 0.32 \\
\hline & 2.931 & -1.48 & 0.544 \\
\hline & 2.468587 & 1.106049 & -0.763002 \\
\hline & 3.6013 & 2.1 & 1.1 \\
\hline & 1.71 & 1.8 & 2.7 \\
\hline & 1.13 & & \\
\hline & 1.164667 & 0.43 & 1.8 \\
\hline & 4.029043 & 0.905889 & 3.232766 \\
\hline & 3.509935 & -0.604772 & 2.508654 \\
\hline & 4.977300 & 0.1 & 1.927539 \\
\hline & -4.781925 & 7364 & -1.682834 \\
\hline & -4.876875 & 2.369260 & 0.040462 \\
\hline & -4.096483 & 3.484713 & -1.09124 \\
\hline & -2.562228 & 1.422985 & -2.739606 \\
\hline & -1.828200 & 2.901072 & -2.103460 \\
\hline & -1.102570 & 1.323475 & -1.740278 \\
\hline & 5.781904 & -0.510251 & -1.931295 \\
\hline & 4.318220 & -1.339308 & -1.508411 \\
\hline
\end{tabular}




\section{Compound 4, conformation e}

\begin{tabular}{|c|c|c|c|}
\hline & -6.511705 & -0.021578 & 0.709475 \\
\hline & -5.524355 & 0.967049 & 0.098503 \\
\hline & -4.506346 & 0.235942 & -0.691708 \\
\hline & -3.682165 & -0.650413 & -0.127304 \\
\hline & -2.889650 & -1.211681 & -1.113286 \\
\hline & -1.765660 & -1.917471 & -0.744128 \\
\hline & -0.75279 & -1.249758 & -0.07 \\
\hline & 0.14812 & -2.101037 & 0.5 \\
\hline & 1.36 & -1.590401 & \\
\hline & 2.230893 & -1.093061 & -0.015524 \\
\hline & 3.252742 & -0.343680 & \\
\hline & 4.17 & 0.37 & -0.3 \\
\hline & 3.99243 & 1.907289 & -0.2 \\
\hline & 2.60 & 2.302941 & -0.7 \\
\hline & 4.264459 & 2.468240 & 1.1 \\
\hline & 5.647532 & -0.019619 & -0.1 \\
\hline & 6.54 & 0.593280 & -0.7 \\
\hline & 2.10 & -1.3757 & -1.1 \\
\hline & -0.66 & -0.03 & -0.0 \\
\hline & -3.62 & -0.973152 & \\
\hline & -6.2018 & 1.830685 & -0.95 \\
\hline & -4.832 & 1.8314 & \\
\hline & 5.872490 & -1.063277 & 0.6 \\
\hline & -6.034513 & -0.64 & 1.4 \\
\hline & -7.328892 & 0.530376 & 1.1 \\
\hline & -6.92 & -0.6643 & -0.0 \\
\hline & -2.8250 & -0.723621 & -1.9 \\
\hline & -1.934587 & -2.889525 & -0.535837 \\
\hline & 0.18 & -3.06 & 0.2 \\
\hline & 1.312931 & -1.120629 & 1.8 \\
\hline & 3.19 & -0.04 & 1.4 \\
\hline & 3.9565 & & $-1 \cdot 3$ \\
\hline & 4.739709 & 2.317364 & -0.97 \\
\hline & 2.493196 & 3.38 & -0.7 \\
\hline & 2.429436 & 1.945989 & -1.81 \\
\hline & 1.820 & $1.88^{\circ}$ & -0.1 \\
\hline & 4.2089 & 3.55 & \\
\hline & 3.5244 & 2.12 & 1.8 \\
\hline & 5.254758 & 2.189163 & 1.4 \\
\hline & -5.480278 & 2.500436 & -1.425108 \\
\hline & -6.651607 & 1.207711 & -1.7 \\
\hline & -6.984746 & 2.430737 & -0.4 \\
\hline & -4.085935 & 2.470162 & 0.6 \\
\hline & -5.573229 & 2.468897 & 1.63102 \\
\hline & -4.348765 & 1.220817 & 1.904 \\
\hline & 6.81483 & -1.390640 & $0 . \varepsilon$ \\
\hline & 5.113153 & -1.578073 & \\
\hline
\end{tabular}




\begin{tabular}{|c|c|c|c|}
\hline $\mathrm{O}$ & 3.776497 & 0.116395 & -1.982659 \\
\hline $\mathrm{C}$ & 3.253150 & 1.050987 & -3.013496 \\
\hline $\mathrm{C}$ & 1.730593 & 1.034945 & -2.998453 \\
\hline C & 3.789384 & 0.440602 & -4.301898 \\
\hline $\mathrm{C}$ & 3.835307 & 2.439127 & -2.775 \\
\hline C & 3.472005 & 0.255554 & -0.696738 \\
\hline 0 & 2.823153 & 1.150390 & -0.185476 \\
\hline $\mathrm{H}$ & .358799 & 1.550592 & -3.883871 \\
\hline $\mathrm{H}$ & 1.373995 & 0.008756 & -3.011638 \\
\hline $\mathrm{H}$ & 1.332493 & 1.525313 & -2.115 \\
\hline $\mathrm{H}$ & 3.490814 & 1.054854 & -5.150467 \\
\hline $\mathrm{H}$ & 3.391114 & -0.563830 & -4.442577 \\
\hline $\mathrm{H}$ & 4.877382 & 0.386331 & -4.2 \\
\hline $\mathrm{H}$ & 3.547508 & 3.0923 & -3.5 \\
\hline $\mathrm{H}$ & 4.923808 & 2.393765 & -2.7 \\
\hline $\mathrm{H}$ & 3.464642 & 2.864943 & -1.8 \\
\hline $\mathrm{N}$ & 3.984295 & -0.8040 & \\
\hline $\mathrm{H}$ & 4.486714 & -1.528213 & -0.4 \\
\hline $\mathrm{N}$ & 4.067693 & -0.781835 & 1.3 \\
\hline C & 5.29 & -0.217081 & 2.0 \\
\hline $\mathrm{H}$ & 5.19 & -0.463700 & \\
\hline $\mathrm{C}$ & 6.527 & -0.908627 & \\
\hline $\mathrm{H}$ & 7.412731 & -0.563749 & \\
\hline $\mathrm{H}$ & 6.45 & -1.990863 & \\
\hline $\mathrm{H}$ & 6.66 & -0.674856 & 0.3 \\
\hline C & 5.3 & 1.301 & \\
\hline $\mathrm{H}$ & 6.233846 & 1.686706 & 2.3 \\
\hline $\mathrm{H}$ & 4.46 & 1.769587 & \\
\hline $\mathrm{H}$ & 5.431592 & 1.589687 & 0.8 \\
\hline C & 2.914045 & -0.912294 & 2.1 \\
\hline 0 & 2.876001 & -0.659024 & 3.3 \\
\hline $\mathrm{N}$ & 1.814402 & -1.358146 & 1.4 \\
\hline $\mathrm{H}$ & 1.812974 & -1.568683 & \\
\hline $\mathrm{N}$ & 0.585666 & -1.359962 & 2.0 \\
\hline $\mathrm{C}$ & 0.232245 & -2.535905 & 2.84 \\
\hline $\mathrm{H}$ & -0.784652 & -2.422831 & 3.2 \\
\hline $\mathrm{H}$ & 0.911107 & -2.647983 & 3.6 \\
\hline $\mathrm{H}$ & 0.291390 & -3.417465 & 2.2 \\
\hline C & -0.132912 & -0.192737 & 2.150 \\
\hline O & -1.207153 & -0.144543 & 2.771 \\
\hline $\mathrm{N}$ & 0.367409 & 0.892362 & 1.5 \\
\hline $\mathrm{H}$ & 1.178526 & 0.808147 & 0.90 \\
\hline C & -0.465369 & 2.073437 & 1.35 \\
\hline $\mathrm{H}$ & -0.796112 & 2.398577 & 2.34 \\
\hline C & 0.323213 & 3.219353 & \\
\hline $\mathrm{H}$ & 0.696643 & 2.846094 & -0.261223 \\
\hline $\mathrm{C}$ & -0.587278 & 4.420568 & 0.42 \\
\hline $\mathrm{H}$ & -1.395937 & 4.181111 & -0.26 \\
\hline $\mathrm{H}$ & -0.012176 & 5.236194 & -0.00 \\
\hline $\mathrm{H}$ & -1.033030 & 4.785597 & \\
\hline $\mathrm{C}$ & 1.517527 & 3.622494 & 1.56 \\
\hline $\mathrm{H}$ & 2.194556 & 2.788784 & 1.73 \\
\hline $\mathrm{H}$ & 1.178535 & 3.997858 & 2.53 \\
\hline $\mathrm{H}$ & 2.082197 & 4.416680 & \\
\hline C & -1.688187 & 1.682041 & 0.51 \\
\hline O & -1.576876 & 1.120574 & -0.567 \\
\hline $\mathrm{N}$ & -2.893082 & 1.946680 & 1.087 \\
\hline $\mathrm{H}$ & -2.929480 & 2.289434 & 2.034 \\
\hline $\mathrm{N}$ & -4.087543 & 1.515408 & 0.537 \\
\hline C & -4.856517 & 2.476676 & -0.2817 \\
\hline $\mathrm{H}$ & -5.818199 & 1.992461 & -0.42987 \\
\hline C & -5.066920 & 3.772284 & 0.4951 \\
\hline $\mathrm{H}$ & -5.536586 & 3.579306 & 1.45 \\
\hline $\mathrm{H}$ & -4.122736 & 4.290824 & 0.66 \\
\hline $\mathrm{H}$ & -5.713952 & 4.437216 & -0.07 \\
\hline
\end{tabular}




$\begin{array}{llrrr}66 & \mathrm{C} & -4.205075 & 2.711662 & -1.643123 \\ 67 & \mathrm{H} & -4.822654 & 3.386115 & -2.237205 \\ 68 & \mathrm{H} & -4.091716 & 1.773790 & -2.183333 \\ 69 & \mathrm{H} & -3.218841 & 3.161643 & -1.531983 \\ 70 & \mathrm{C} & -4.337496 & 0.154933 & 0.448947 \\ 71 & \mathrm{O} & -5.232495 & -0.298780 & -0.250395 \\ 72 & \mathrm{~N} & -3.516925 & -0.637267 & 1.218353 \\ 73 & \mathrm{H} & -2.783453 & -0.283524 & 1.829724 \\ 74 & \mathrm{~N} & -3.568956 & -2.006857 & 1.088999 \\ 75 & \mathrm{C} & -4.491830 & -2.724576 & 1.952082 \\ 76 & \mathrm{H} & -4.388965 & -3.787132 & 1.761446 \\ 77 & \mathrm{H} & -5.516729 & -2.414788 & 1.744613 \\ 78 & \mathrm{H} & -4.258029 & -2.519313 & 2.997136 \\ 79 & \mathrm{C} & -2.824873 & -2.635817 & 0.112904 \\ 80 & \mathrm{O} & -2.843910 & -3.865117 & -0.022296 \\ 81 & \mathrm{~N} & -2.074067 & -1.824802 & -0.676976 \\ 82 & \mathrm{H} & -2.026590 & -0.828732 & -0.515462 \\ 83 & \mathrm{C} & -1.278314 & -2.353917 & -1.773815 \\ 84 & \mathrm{H} & -1.757595 & -3.272467 & -2.112869 \\ 85 & \mathrm{C} & -1.209618 & -1.362404 & -2.940510 \\ 86 & \mathrm{H} & -0.715796 & -0.462698 & -2.573047 \\ 87 & \mathrm{C} & -0.387021 & -1.946355 & -4.091898 \\ 88 & \mathrm{H} & 0.627418 & -2.193220 & -3.782561 \\ 89 & \mathrm{H} & -0.320930 & -1.229504 & -4.910751 \\ 90 & \mathrm{H} & -0.857629 & -2.853025 & -4.479881 \\ 91 & \mathrm{C} & -2.614251 & -0.985877 & -3.420048 \\ 92 & \mathrm{H} & -2.549549 & -0.272371 & -4.242352 \\ 93 & \mathrm{H} & -3.149046 & -1.867272 & -3.782591 \\ 94 & \mathrm{H} & -3.208915 & -0.533495 & -2.628808 \\ 95 & \mathrm{C} & 0.102026 & -2.707283 & -1.192953 \\ 96 & \mathrm{O} & 1.060190 & -1.933072 & -1.235689 \\ 97 & \mathrm{~N} & 0.154773 & -3.907747 & -0.589909 \\ 98 & \mathrm{H} & -0.701425 & -4.415126 & -0.416663 \\ 99 & \mathrm{H} & 0.986928 & -4.169701 & -0.086526\end{array}$




\begin{tabular}{|c|c|c|c|}
\hline & -6.110056 & 0.717578 & -0.399788 \\
\hline & -6.383647 & 2.180840 & -0.326396 \\
\hline & -7.879942 & 2.243002 & -0.600254 \\
\hline & -5.592599 & 2.889529 & -1.418726 \\
\hline & -6.059244 & 2.698446 & 1.070204 \\
\hline & -4.889521 & 0.227837 & -0.231916 \\
\hline & -3.89302 & .827478 & \\
\hline & -8.43126 & 1.688999 & \\
\hline & -8.21132 & 3.280428 & -0.582 \\
\hline & -8.108955 & 1.821018 & -1.578165 \\
\hline & -5.814510 & 2.451835 & -2.391864 \\
\hline & -4.523 & 2.827 & -1.23 \\
\hline & -5.8 & 3.94 & -1.44 \\
\hline & -6.41 & 3.725 & \\
\hline & -6.568225 & 2.096535 & \\
\hline & -4.991023 & 2.679666 & \\
\hline & -4.873 & -1.10 & -0.5 \\
\hline & -5.73 & -1.55 & -0.8 \\
\hline & -3.82 & -1.92 & -0.2 \\
\hline & -3.885919 & -2.593869 & \\
\hline & -3.020804 & -3.250512 & \\
\hline & -3.762990 & -1.594347 & \\
\hline & -3.71 & -2.13 & 3.2 \\
\hline & -4.62 & -0.92 & \\
\hline & -2.863 & -0.991 & 2.1 \\
\hline & -5.15 & & \\
\hline & -6.047 & -2.813 & 1.2 \\
\hline & -5.220 & -4.140879 & 0.3 \\
\hline & -5.14 & -4.002485 & 2.1 \\
\hline & -2.62 & -1.784 & -0.8 \\
\hline & -1.58 & -2.27 & -0.4 \\
\hline & -2.640 & -1.039063 & $-2 \cdot 0$ \\
\hline & -3.482 & -0.614638 & $-2 \cdot 3$ \\
\hline & -1.45 & -0.692 & -2 \\
\hline & -0.91 & -1.656812 & -3.5 \\
\hline & -0.102 & -1.196831 & $-4 \cdot 1$ \\
\hline & -0.559 & -2.555147 & -3.0 \\
\hline & -1.715 & -1.918818 & -4.2 \\
\hline & -0.56 & 0.106093 & -1.9 \\
\hline & 0.627 & 0.161738 & -2.23 \\
\hline & -1.066832 & 0.799863 & -0.86 \\
\hline & -2.046 & 0.738616 & -0.6 \\
\hline & -0.167590 & 1.484929 & \\
\hline & 0.413230 & 2.234062 & -0.4 \\
\hline & -0.992627 & 2.193003 & 1.1 \\
\hline & -1.691811 & 1.454988 & 1.5 \\
\hline & -0.121 & & \\
\hline & -0.742 & & \\
\hline & 0.650 & 3.384152 & 1.92 \\
\hline & 0.371653 & 1.899747 & 2.83 \\
\hline & -1.797120 & 3.343737 & 0.53 \\
\hline & -2.449 & 3.787208 & \\
\hline & -1.124840 & 4.121839 & \\
\hline & -2.423401 & 3.012782 & -0.289356 \\
\hline & 0.894 & 0.545121 & 0.62 \\
\hline & 2.006009 & 0.973513 & 0.92 \\
\hline & 0.535249 & -0.750830 & 0.780089 \\
\hline & -0.342300 & -1.114398 & 0.415266 \\
\hline & 1.428589 & -1.709854 & 1.22 \\
\hline & 1.369786 & -2.100529 & 2.650365 \\
\hline & 1.911420 & -3.042007 & 2.69 \\
\hline & 2.073956 & -1.080681 & 3.542976 \\
\hline & 2.045156 & -1.410249 & \\
\hline & 3.114220 & -0.965870 & 3.24378 \\
\hline & 1.586 & -0.108093 & 3.4 \\
\hline
\end{tabular}




$\begin{array}{llrrr}66 & \mathrm{C} & -0.077266 & -2.338864 & 3.068411 \\ 67 & \mathrm{H} & -0.098777 & -2.760005 & 4.072763 \\ 68 & \mathrm{H} & -0.575569 & -3.030723 & 2.389773 \\ 69 & \mathrm{H} & -0.642344 & -1.407290 & 3.084827 \\ 70 & \mathrm{C} & 2.517658 & -2.049106 & 0.449953 \\ 71 & \mathrm{O} & 3.446634 & -2.713380 & 0.892322 \\ 72 & \mathrm{~N} & 2.488272 & -1.603219 & -0.853149 \\ 73 & \mathrm{H} & 1.730881 & -1.067586 & -1.263657 \\ 74 & \mathrm{~N} & 3.601365 & -1.786382 & -1.650501 \\ 75 & \mathrm{C} & 3.753669 & -3.103580 & -2.256192 \\ 76 & \mathrm{H} & 3.978378 & -3.860788 & -1.502617 \\ 77 & \mathrm{H} & 4.559012 & -3.066443 & -2.981827 \\ 78 & \mathrm{H} & 2.823512 & -3.358425 & -2.759920 \\ 79 & \mathrm{C} & 4.721876 & -0.991238 & -1.426501 \\ 80 & \mathrm{O} & 5.817202 & -1.295685 & -1.894838 \\ 81 & \mathrm{~N} & 4.496307 & 0.140603 & -0.703465 \\ 82 & \mathrm{H} & 3.554859 & 0.354233 & -0.416422 \\ 83 & \mathrm{C} & 5.564396 & 1.062633 & -0.367882 \\ 84 & \mathrm{H} & 6.478206 & 0.628706 & -0.763990 \\ 85 & \mathrm{C} & 5.364742 & 2.444787 & -1.050375 \\ 86 & \mathrm{H} & 5.189185 & 2.198474 & -2.101249 \\ 87 & \mathrm{C} & 4.141232 & 3.202509 & -0.529640 \\ 88 & \mathrm{H} & 4.277517 & 3.496941 & 0.512752 \\ 89 & \mathrm{H} & 3.229681 & 2.609326 & -0.587314 \\ 90 & \mathrm{H} & 3.988368 & 4.110403 & -1.114416 \\ 91 & \mathrm{C} & 6.622631 & 3.311627 & -0.971372 \\ 92 & \mathrm{H} & 6.481215 & 4.227337 & -1.547321 \\ 93 & \mathrm{H} & 7.492279 & 2.788769 & -1.372022 \\ 94 & \mathrm{H} & 6.846223 & 3.588225 & 0.058684 \\ 95 & \mathrm{C} & 5.807099 & 1.189336 & 1.143580 \\ 96 & \mathrm{O} & 6.931281 & 1.463344 & 1.558992 \\ 97 & \mathrm{~N} & 4.755774 & 1.013617 & 1.966695 \\ 98 & \mathrm{H} & 3.808319 & 0.866257 & 1.647290 \\ 99 & \mathrm{H} & 4.906657 & 1.147052 & 2.952866\end{array}$


Compound 5 , conformation $5 \mathrm{C}+/+I-I-I-I-$ (from NMR parameters-restrained molecular dynamics)

\begin{tabular}{|c|c|c|c|}
\hline 0 & 5.238902 & 1.304696 & -0.566744 \\
\hline $\mathrm{C}$ & 4.897541 & 2.663588 & -0.065107 \\
\hline C & 5.842927 & 3.549473 & -0.865170 \\
\hline C & 3.442387 & 2.982158 & -0.389927 \\
\hline C & 5.196788 & 2.744043 & 1.427259 \\
\hline C & 4.584806 & 0.225845 & -0.150198 \\
\hline O & 3.724109 & 0.172094 & 0.709375 \\
\hline $\mathrm{H}$ & 5.648287 & 3.451751 & -1.932709 \\
\hline $\mathrm{H}$ & 5.699105 & 4.590540 & -0.579055 \\
\hline $\mathrm{H}$ & 6.879397 & 3.275434 & -0.671437 \\
\hline $\mathrm{H}$ & 3.240236 & 2.807286 & -1.446593 \\
\hline $\mathrm{H}$ & 3.252122 & 4.033283 & -0.176037 \\
\hline $\mathrm{H}$ & 2.761720 & 2.381976 & 0.206001 \\
\hline $\mathrm{H}$ & 6.226085 & 2.443703 & 1.622797 \\
\hline $\mathrm{H}$ & 5.071139 & 3.774342 & 1.759964 \\
\hline $\mathrm{H}$ & 4.526479 & 2.108223 & 1.998495 \\
\hline $\mathrm{N}$ & 4.985955 & -0.867624 & -0.882419 \\
\hline $\mathrm{H}$ & 5.698171 & -0.765275 & -1.587631 \\
\hline $\mathrm{N}$ & 4.588704 & -2.144941 & -0.544740 \\
\hline C & 5.457026 & -2.930627 & 0.362431 \\
\hline $\mathrm{H}$ & 5.003556 & -3.917960 & 0.374875 \\
\hline C & 6.862115 & -3.035065 & -0.222042 \\
\hline $\mathrm{H}$ & 7.464635 & -3.699344 & 0.396421 \\
\hline $\mathrm{H}$ & 6.837262 & -3.435988 & -1.235377 \\
\hline $\mathrm{H}$ & 7.357841 & -2.063391 & -0.241945 \\
\hline $\mathrm{C}$ & 5.459155 & -2.363872 & 1.780308 \\
\hline 27 & 4.449491 & -2.319556 & 2.183341 \\
\hline $\mathrm{H}$ & 5.881763 & -1.358935 & 1.800711 \\
\hline $\mathrm{H}$ & 6.066807 & -2.997099 & 2.427071 \\
\hline C & 3.270387 & -2.503965 & -0.737141 \\
\hline 31 & 2.769176 & -3.496718 & -0.232538 \\
\hline 32 & 2.547721 & -1.640376 & -1.535704 \\
\hline $\mathrm{H}$ & 2.988512 & -0.900441 & -2.055740 \\
\hline $\mathrm{N}$ & 1.197932 & -1.819784 & -1.709399 \\
\hline C & 0.766334 & -2.746457 & -2.747885 \\
\hline $\mathrm{H}$ & 1.106976 & -3.755779 & -2.515622 \\
\hline 37 & -0.316478 & -2.729231 & -2.799894 \\
\hline 38 & 1.181115 & -2.433842 & -3.705209 \\
\hline C & 0.328732 & -1.323620 & -0.764820 \\
\hline 40 & -0.893579 & -1.501483 & -0.858491 \\
\hline 41 & 0.867956 & -0.591000 & 0.244349 \\
\hline 42 & 1.868370 & -0.457504 & 0.314884 \\
\hline 43 & 0.023229 & -0.113075 & 1.328695 \\
\hline 44 & -0.567685 & -0.941205 & 1.723346 \\
\hline 45 & 0.904944 & 0.448754 & 2.469250 \\
\hline 46 & 1.609906 & 1.152645 & 2.020063 \\
\hline 47 & 0.086959 & 1.193252 & 3.525763 \\
\hline 48 & 0.744615 & 1.517731 & 4.332308 \\
\hline 49 & -0.681130 & 0.547048 & 3.953371 \\
\hline 50 & -0.407913 & 2.074526 & 3.121939 \\
\hline 51 & 1.700894 & -0.688625 & 3.118591 \\
\hline 52 & 2.294780 & -1.241673 & 2.394997 \\
\hline 53 & 1.024225 & -1.386922 & 3.615857 \\
\hline 54 & 2.383395 & -0.287316 & 3.868033 \\
\hline 55 & -1.027571 & 0.889253 & 0.841469 \\
\hline 56 & -2.122802 & 0.981623 & 1.369508 \\
\hline 57 & -0.654716 & 1.645892 & -0.229179 \\
\hline 58 & 0.226442 & 1.466460 & -0.684012 \\
\hline 59 & -1.517441 & 2.522166 & -0.860825 \\
\hline 60 & -1.451814 & 3.948195 & -0.475217 \\
\hline 61 & -2.042083 & 4.457947 & -1.231972 \\
\hline 62 & -2.079540 & 4.194591 & 0.894964 \\
\hline 63 & -2.084395 & 5.262874 & 1.114530 \\
\hline 64 & -1.515149 & 3.687483 & 1.677049 \\
\hline 65 & -3.103020 & 3.825080 & 0.920006 \\
\hline
\end{tabular}




$\begin{array}{llrrr}66 & \mathrm{C} & -0.011177 & 4.442149 & -0.549998 \\ 67 & \mathrm{H} & 0.421605 & 4.250802 & -1.532177 \\ 68 & \mathrm{H} & 0.611991 & 3.958544 & 0.202246 \\ 69 & \mathrm{H} & 0.015830 & 5.515479 & -0.365125 \\ 70 & \mathrm{C} & -2.634590 & 2.023638 & -1.510668 \\ 71 & \mathrm{O} & -3.532506 & 2.754356 & -1.906780 \\ 72 & \mathrm{~N} & -2.642386 & 0.662559 & -1.701068 \\ 73 & \mathrm{H} & -1.890912 & 0.038734 & -1.420337 \\ 74 & \mathrm{~N} & -3.704457 & 0.078085 & -2.351853 \\ 75 & \mathrm{C} & -3.616272 & -0.012342 & -3.800262 \\ 76 & \mathrm{H} & -2.797552 & -0.669783 & -4.104172 \\ 77 & \mathrm{H} & -4.551268 & -0.405174 & -4.184037 \\ 78 & \mathrm{H} & -3.447452 & 0.983667 & -4.206254 \\ 79 & \mathrm{C} & -4.627204 & -0.660480 & -1.631765 \\ 80 & \mathrm{O} & -5.476583 & -1.342449 & -2.208882 \\ 81 & \mathrm{~N} & -4.527715 & -0.574362 & -0.277790 \\ 82 & \mathrm{H} & -3.861330 & 0.052452 & 0.145852 \\ 83 & \mathrm{C} & -5.400467 & -1.330077 & 0.589007 \\ 84 & \mathrm{H} & -6.153914 & -1.784121 & -0.052636 \\ 85 & \mathrm{C} & -4.661464 & -2.476650 & 1.337698 \\ 86 & \mathrm{H} & -5.433303 & -3.048244 & 1.859870 \\ 87 & \mathrm{C} & -3.973567 & -3.408249 & 0.337279 \\ 88 & \mathrm{H} & -4.673941 & -3.772528 & -0.415258 \\ 89 & \mathrm{H} & -3.160371 & -2.892935 & -0.175185 \\ 90 & \mathrm{H} & -3.550310 & -4.269450 & 0.856101 \\ 91 & \mathrm{C} & -3.667176 & -1.953191 & 2.375607 \\ 92 & \mathrm{H} & -3.156239 & -2.785707 & 2.860882 \\ 93 & \mathrm{H} & -2.913715 & -1.318080 & 1.911380 \\ 94 & \mathrm{H} & -4.160753 & -1.365956 & 3.151035 \\ 95 & \mathrm{C} & -6.106730 & -0.390589 & 1.574476 \\ 96 & \mathrm{O} & -5.709375 & 0.744963 & 1.803112 \\ 97 & \mathrm{~N} & -7.177297 & -0.925380 & 2.200094 \\ 98 & \mathrm{H} & -7.531151 & -1.838120 & 1.975198 \\ 99 & \mathrm{H} & -7.670236 & -0.378067 & 2.885454\end{array}$




\begin{tabular}{|c|c|c|c|}
\hline O & 5.240668 & 0.922583 & 0.074672 \\
\hline C & 5.596914 & 2.082732 & -0.785062 \\
\hline C & 5.660453 & 1.628935 & -2.238872 \\
\hline C & 4.601051 & 3.213801 & -0.564928 \\
\hline C & 6.978219 & 2.457943 & -0.265241 \\
\hline C & 4.086632 & 0.276384 & -0.056389 \\
\hline O & .1746 & 0.5437 & -0.815598 \\
\hline $\mathrm{H}$ & 6.3317 & 0.77 & -2.339912 \\
\hline $\mathrm{H}$ & 4.67 & 1.3525 & -2.609536 \\
\hline $\mathrm{H}$ & 6.047333 & 2.444403 & -2.849761 \\
\hline $\mathrm{H}$ & 3.618794 & 2.956 & -0.948132 \\
\hline $\mathrm{H}$ & 4.51 & 3.44 & 0.496815 \\
\hline $\mathrm{H}$ & 4.95 & 4.10 & -1.081260 \\
\hline $\mathrm{H}$ & 7.36 & 3.30 & -0.829426 \\
\hline $\mathrm{H}$ & 7.669329 & 1.623155 & -0.376971 \\
\hline $\mathrm{H}$ & 6.929297 & 2.73 & 0.787709 \\
\hline $\mathrm{N}$ & 4.04 & -0.75 & 0.861300 \\
\hline $\mathrm{H}$ & 4.88 & -0.97 & 1.369944 \\
\hline $\mathrm{N}$ & 3.10 & $-1 \cdot 7$ & 0.7 \\
\hline C & 3.48 & -2.999990 & 0.007465 \\
\hline $\mathrm{H}$ & 2.53 & -3.461484 & -0.259714 \\
\hline C & 4.24 & -2.68 & -1.275145 \\
\hline $\mathrm{H}$ & 5.19 & -2.19 & -1.071830 \\
\hline $\mathrm{H}$ & 3.64 & -2.04 & -1.924273 \\
\hline $\mathrm{H}$ & 4.44 & -3.61 & -1.802832 \\
\hline C & 4.26 & -3.9 & 0.9 \\
\hline $\mathrm{H}$ & 3.689436 & -4.15 & 1.8 \\
\hline $\mathrm{H}$ & 5.212757 & -3.4 & 1.2 \\
\hline $\mathrm{H}$ & 4.505612 & -4.85 & 0.43 \\
\hline C & 1.90 & -1.6 & 1.3 \\
\hline O & 1.025 & -2.49 & 8753 \\
\hline $\mathrm{N}$ & 1.748 & -0.46 & 2.0 \\
\hline $\mathrm{H}$ & 2.570138 & -0.01 & 2.4 \\
\hline $\mathrm{N}$ & 0.56 & -0.2 & $2.7 \varepsilon$ \\
\hline $\mathrm{C}$ & 0.303 & -1.0 & 3.97 \\
\hline $\mathrm{H}$ & -0.676 & -0.79 & 4.35 \\
\hline $\mathrm{H}$ & 0.320 & -2.12 & 3.7 \\
\hline $\mathrm{H}$ & 1.057314 & -0.85 & 4.7 \\
\hline C & -0.271933 & 0.722 & 2.352968 \\
\hline O & -1.361 & 0.93 & 2.9 \\
\hline $\mathrm{N}$ & 0.155723 & 1.47 & 1.30 \\
\hline $\mathrm{H}$ & 1.000004 & 1.20 & 0.8 \\
\hline C & -0.667447 & 2.54 & 0.7 \\
\hline $\mathrm{H}$ & -1.013880 & 3.15 & 1.603270 \\
\hline C & 0.139414 & 3.432121 & -0.192780 \\
\hline $\mathrm{H}$ & 0.553521 & 2.780752 & -0.967641 \\
\hline C & -0.769407 & 4.463173 & -0.8 \\
\hline $\mathrm{H}$ & -1.538972 & 3.99 & -1.478608 \\
\hline $\mathrm{H}$ & -1.261995 & 5.092634 & -0.122385 \\
\hline $\mathrm{H}$ & -0.181858 & 5.110978 & -1.516238 \\
\hline C & 1.285000 & 4.122554 & 0.550802 \\
\hline $\mathrm{H}$ & 1.876119 & 4.720 & -0.141964 \\
\hline $\mathrm{H}$ & 1.955308 & 3.411 & 1.029616 \\
\hline $\mathrm{H}$ & 0.891514 & 4.788 & 1.321589 \\
\hline C & -1.872300 & 1.913163 & 0.064821 \\
\hline O & -1.738759 & 1.173428 & -0.902948 \\
\hline $\mathrm{N}$ & -3.073677 & 2.179170 & 0.633419 \\
\hline $\mathrm{H}$ & -3.099526 & 2.670066 & 1.514127 \\
\hline $\mathrm{N}$ & -4.236223 & 1.531594 & 0.251740 \\
\hline C & -5.149851 & 2.245560 & -0.665816 \\
\hline $\mathrm{H}$ & -6.048478 & 1.634365 & -0.676042 \\
\hline C & -5.487803 & 3.620408 & -0.098877 \\
\hline $\mathrm{H}$ & -6.230791 & 4.102613 & -0.733079 \\
\hline $\mathrm{H}$ & -4.607064 & 4.263142 & -0.068778 \\
\hline $\mathrm{H}$ & -5.894722 & 3.539782 & 0.909239 \\
\hline
\end{tabular}




$\begin{array}{llrrr}66 & \mathrm{C} & -4.582785 & 2.320833 & -2.081466 \\ 67 & \mathrm{H} & -5.299516 & 2.808918 & -2.742486 \\ 68 & \mathrm{H} & -4.374601 & 1.324534 & -2.466405 \\ 69 & \mathrm{H} & -3.657164 & 2.896189 & -2.099856 \\ 70 & \mathrm{C} & -4.315381 & 0.155265 & 0.402433 \\ 71 & \mathrm{O} & -5.169457 & -0.514082 & -0.160894 \\ 72 & \mathrm{~N} & -3.352539 & -0.391385 & 1.220043 \\ 73 & \mathrm{H} & -2.762404 & 0.160755 & 1.836167 \\ 74 & \mathrm{~N} & -3.268025 & -1.757671 & 1.370546 \\ 75 & \mathrm{C} & -4.172690 & -2.373722 & 2.329553 \\ 76 & \mathrm{H} & -3.947018 & -3.432424 & 2.388485 \\ 77 & \mathrm{H} & -4.025276 & -1.918340 & 3.308413 \\ 78 & \mathrm{H} & -5.210505 & -2.237626 & 2.020370 \\ 79 & \mathrm{C} & -2.578101 & -2.513475 & 0.430049 \\ 80 & \mathrm{O} & -2.534868 & -3.739830 & 0.522291 \\ 81 & \mathrm{~N} & -1.957751 & -1.807058 & -0.553844 \\ 82 & \mathrm{H} & -2.113646 & -0.813692 & -0.612651 \\ 83 & \mathrm{C} & -1.209650 & -2.465411 & -1.607496 \\ 84 & \mathrm{H} & -1.125216 & -3.507900 & -1.314441 \\ 85 & \mathrm{C} & -1.931900 & -2.418572 & -2.981036 \\ 86 & \mathrm{H} & -1.288002 & -2.974196 & -3.667216 \\ 87 & \mathrm{C} & -3.283476 & -3.130438 & -2.897486 \\ 88 & \mathrm{H} & -3.759897 & -3.158280 & -3.878438 \\ 89 & \mathrm{H} & -3.953294 & -2.605982 & -2.214057 \\ 90 & \mathrm{H} & -3.173518 & -4.154827 & -2.540335 \\ 91 & \mathrm{C} & -2.097924 & -0.994722 & -3.518472 \\ 92 & \mathrm{H} & -1.139157 & -0.491230 & -3.641202 \\ 93 & \mathrm{H} & -2.706208 & -0.388240 & -2.846695 \\ 94 & \mathrm{H} & -2.594728 & -1.015210 & -4.489182 \\ 95 & \mathrm{C} & 0.239845 & -1.983775 & -1.751222 \\ 96 & \mathrm{O} & 1.075883 & -2.756170 & -2.221774 \\ 97 & \mathrm{~N} & 0.530637 & -0.722372 & -1.400554 \\ 98 & \mathrm{H} & -0.173731 & -0.074226 & -1.081130 \\ 99 & \mathrm{H} & 1.494568 & -0.414610 & -1.399738\end{array}$

